\title{
Radical Substitution Provides a Unique Route to Disulfides
}

\author{
Zijun Wu and Derek A. Pratt* \\ Department of Chemistry and Biomolecular Sciences, University of Ottawa \\ Ottawa, ON K1N 6N5, Canada \\ dpratt@uottawa.ca
}

Supporting Information

Table of Contents

Section

Page

1. Materials and Methods $\quad$ S2

2. Reaction Optimization and Experimental Procedures S2

2.1 General Information $\quad$ S2

2.2 Initial Exploration with AIBN and Lauroyl Peroxide as Radical Precursors $\quad$ S2

2.3 Optimization of Conditions with PTOC Esters as the Substrates S3

2.4 Optimization of Conditions with Oxime Esters as the Substrates S4

3. Control Experiments and Mechanistic Studies S5

4. Additional Synthetic Transformations $\quad$ S11

5. Characterization Data $\quad$ S14

6. Computational Details $\quad$ S25

7. References $\quad$ S33

$\begin{array}{lr}\text { 8. NMR Spectra } & \text { S34 }\end{array}$

1. Materials and Methods 
All chemicals were purchased from commercial sources and used without further purification, except where otherwise stated. Anhydrous ethyl acetate (EtOAc) was distilled from calcium hydride $(10 \% \mathrm{w} / \mathrm{v})$ under positive pressure of nitrogen and stored over molecular sieves. All other anhydrous solvents were purchased from Fisher Chemicals, Sigma Aldrich or Acros Organics and used without further purification, unless otherwise stated. All reactions were carried out under positive pressure of nitrogen using standard Schlenk technique, unless otherwise noted. Reactions were monitored by thin layer chromatography (TLC) with precoated silica gel plates from Merck Chemicals (TLC Silica gel 60 F254, $250 \mu \mathrm{m}$ thickness) using UV light as the visualizing agent and/or iodine vapor, basic aqueous potassium permanganate $\left(\mathrm{KMnO}_{4}\right)$, or heat as developing agents. Flash column chromatography was performed over silica gel 60 (particle size $0.035-0.07 \mathrm{~mm}$ ) from Silicycle. NMR spectra were recorded on either Bruker AV-300, AV-400 or AV-600 unity plus spectrometers. Chemical shifts $(\delta)$ are quoted in parts per million (ppm) and were internally referenced to residual $\mathrm{CHCl}_{3}(7.26 \mathrm{ppm}$ for ${ }^{1} \mathrm{H}, 77.16$ ppm for ${ }^{13} \mathrm{C}$ ). Coupling constants $(J)$ are reported in Hertz $(\mathrm{Hz})$ to the nearest $0.1 \mathrm{~Hz}$. The following abbreviations (or combinations thereof) were used for peak multiplicities: $s=$ singlet, $d=$ doublet, $t=$ triplet, $q$ = quartet, quintet, $\mathrm{m}=$ multiplet.

\section{Reaction Optimization and Experimental Procedures}

\subsection{General Information}

$\left.\left[\operatorname{Ir}\left(\mathrm{dF}\left(\mathrm{CF}_{3}\right) \mathrm{ppy}\right)_{2}(\mathrm{dtbbpy})\right]\left(\mathrm{PF}_{6}\right)\right], \mathrm{dF}\left(\mathrm{CF}_{3}\right) \mathrm{ppy}=$ 2-(2,4-difluorophenyl)- 3-trifluoromethylpyridine, dtbbpy = 4,4'di-tert-butyl-2,2'-bipyridine) was prepared according to the reported literature procedure ${ }^{1}$. Barton PTOC esters, ${ }^{2}$ oxime esters, ${ }^{3}$ and tetrasulfides ${ }^{4,5}$ were also prepared according to the reported literature procedure. The light source was placed about $7 \mathrm{~cm}$ from the reaction vessel. A custom made photoreactor was used with 4 LEDs $\left(455 \mathrm{~nm}\right.$ ) or UV-A LEDs $\left(\lambda_{\max }=365 \mathrm{~nm}\right.$ ) arranged around the reaction vessel at room temperature.

\subsection{Initial Exploration with AIBN and Lauroyl Peroxide as Radical Precursors}

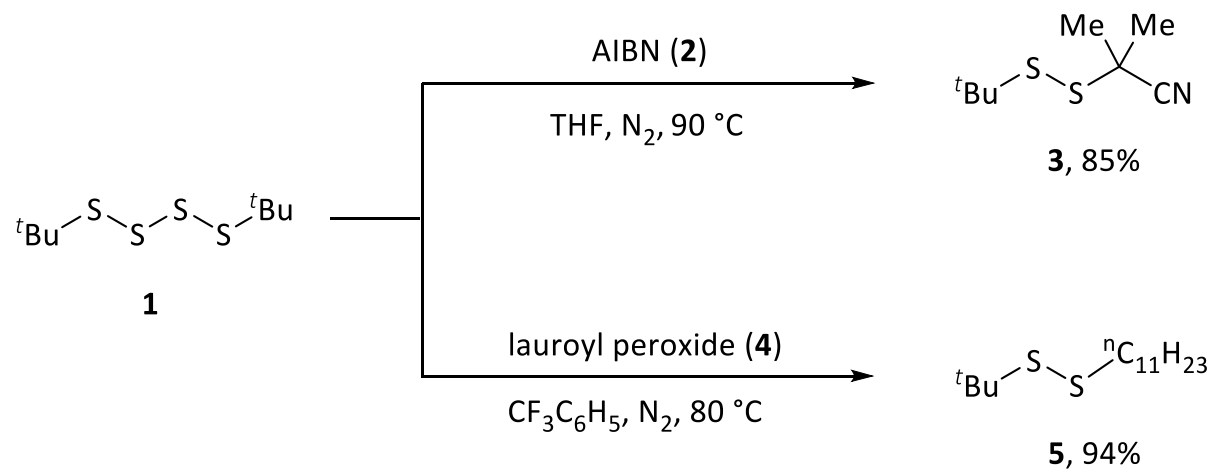

Synthetic procedure A: To an oven dried Schlenk tube with a magnetic stirring bar was added AIBN (2, 98.8 $\mathrm{mg}, 0.6 \mathrm{mmol}),{ }^{t} \mathrm{BuSSSS}{ }^{t} \mathrm{Bu}(\mathbf{1}, 48.5 \mathrm{mg}, 0.2 \mathrm{mmol})$ and $2.0 \mathrm{~mL}$ anhydrous THF under nitrogen atmosphere and the resulting solution was degassed by three freeze-pump-thaw cycles. After back filling with nitrogen, the tube was heated to $90^{\circ} \mathrm{C}$ with stirring for 12 hours. The solvent was removed under reduced pressure and the 
crude residue was purified by column chromatography with hexanes as the eluent to afford the desired product 3 (64.4 mg, 85\% yield).

Synthetic procedure B: To an oven dried Schlenk tube with a magnetic stirring bar was added lauroyl peroxide $(4,79.7 \mathrm{mg}, 0.2 \mathrm{mmol}),{ }^{t} \mathrm{BuSSSS}{ }^{t} \mathrm{Bu}(1,24.2 \mathrm{mg}, 0.1 \mathrm{mmol})$ and $1.0 \mathrm{~mL} \mathrm{CF}_{3} \mathrm{C}_{6} \mathrm{H}_{5}$ under nitrogen atmosphere and the resulting solution was degassed by three freeze-pump-thaw cycles. After back filling with nitrogen, the tube was heated to $80^{\circ} \mathrm{C}$ with stirring for 12 hours. The solvent was removed under reduced pressure and the crude residue was purified by column chromatography with hexanes as the eluent to afford the desired product 5 (22.8 $\mathrm{mg}, 94 \%$ yield).

\subsection{Optimization of Conditions with PTOC Esters as the Substrates}

\section{Table S1.}

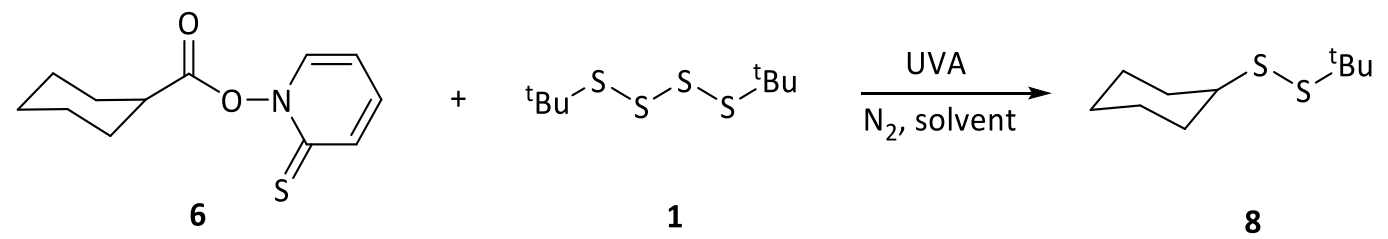

\begin{tabular}{cccc}
\hline Entry & Solvent & Ratio $(\mathbf{6}: \mathbf{1})$ & Yield of 8 (\%) \\
\hline 1 & THF & $2: 1$ & 79 \\
2 & DCM & $2: 1$ & 75 \\
3 & DMF & $2: 1$ & trace \\
4 & $\mathrm{CH}_{3} \mathrm{CN}$ & $2: 1$ & 40 \\
5 & Benzene & $2: 1$ & 65 \\
6 & $\mathrm{CHCl}_{3}$ & $2: 1$ & 72 \\
7 & $\mathrm{TBME}$ & $2: 1$ & trace \\
8 & $\mathrm{THF}$ & $2: 1$ & trace \\
9 & $\mathrm{THF}$ & $2.5: 1$ & $93(90)^{\mathrm{c}}$
\end{tabular}

a. Product determined relative to 1,3,5-trimethoxybenzene as internal standard. b. No irradiation. c. Isolated yield. Reaction conditions: To an oven dried Schlenk tube with a magnetic stirring bar was added PTOC ester $(6,59.3 \mathrm{mg}, 0.25 \mathrm{mmol}),{ }^{{ }^{t} \mathrm{BuSSSS}}{ }^{\mathrm{t}} \mathrm{Bu}(\mathbf{1}, 24.2 \mathrm{mg}, 0.1 \mathrm{mmol})$ and $1.0 \mathrm{~mL}$ anhydrous solvent under nitrogen atmosphere and the resulting solution was degassed by three freeze-pump-thaw cycles. After backfilling with nitrogen, the tube was placed in a photoreactor, stirred and irradiated with UVA for 12-14 hours. For entry 19, $33.6 \mathrm{mg}$ 1,3,5-trimethoxybenzene was added to the reaction tubes as the internal standard to determine the yield. The solvent was removed under reduced pressure and the crude residue was purified by column chromatography with hexanes as the eluent to afford the desired product 8 ( $36.8 \mathrm{mg}, 90 \%$ yield). 


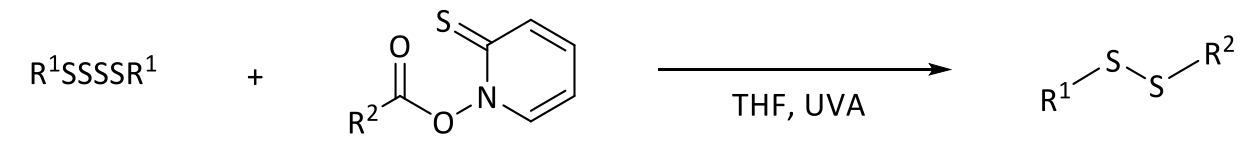

tetrasulfide

$0.1 \mathrm{mmol}$

PTOC

$0.25 \mathrm{mmol}$

General synthetic procedure C: To an oven dried Schlenk tube with a magnetic stirring bar was added PTOC ester $(0.25 \mathrm{mmol})$, tetrasulfide $(0.1 \mathrm{mmol})$ and $1.0 \mathrm{~mL}$ anhydrous THF under nitrogen atmosphere and the resulting solution was degassed by three freeze-pump-thaw cycles. After backfilling with nitrogen, the tube was placed in a photoreactor, stirred and irradiated with UVA for 12-14 hours. The solvent was removed under reduced pressure and the crude residue was purified by column chromatography with hexanes as the eluent to afford the desired disulfide product.

\subsection{Optimization of Conditions with Oxime Esters as the Substrates}

Table S2
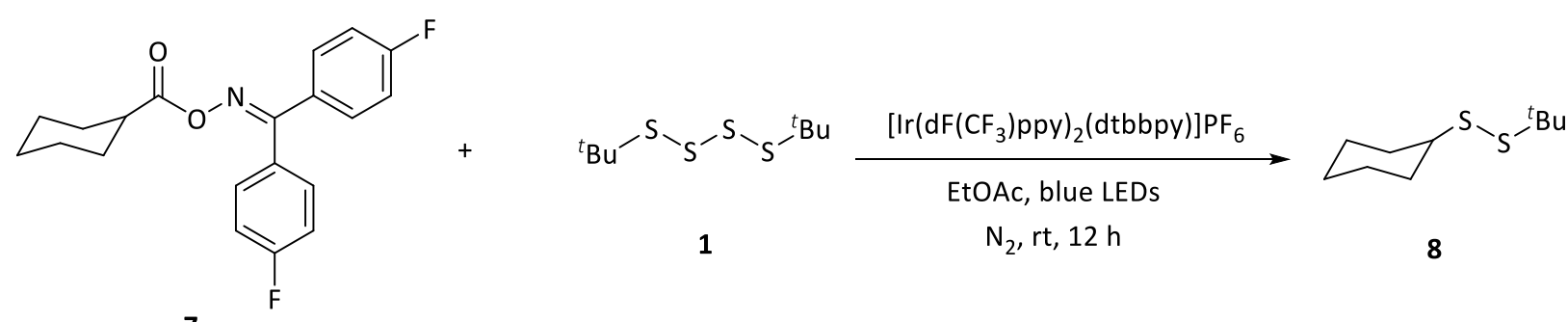

7

\begin{tabular}{|c|c|c|}
\hline Entry & Variations from the standard condition & Yield of $8(\%)^{a}$ \\
\hline 1 & none & $93(87)^{b}$ \\
\hline 2 & $\mathrm{Acr}^{+} \mathrm{MesClO} 4^{-}$as the catalyst & No reaction \\
\hline 3 & {$\left[\operatorname{Ir}\left(\mathrm{dF}(\mathrm{ppy})_{2}(\mathrm{dtbbpy})\right] \mathrm{PF}_{6}\right.$ as catalyst } & No reaction \\
\hline 4 & {$\left[\mathrm{Ru}(\mathrm{bpy})_{3}\right]\left(\mathrm{PF}_{6}\right)_{2}$ as catalyst } & No reaction \\
\hline 5 & Under air & 18 \\
\hline 6 & DCM instead of EtOAC & 76 \\
\hline 7 & THF instead of EtOAc & 73 \\
\hline 8 & $\mathrm{CH}_{3} \mathrm{CN}$ instead of EtOAc & 67 \\
\hline 9 & Without catalyst & No reaction \\
\hline 10 & Without Blue LEDs & No reaction \\
\hline \multirow[t]{2}{*}{11} & $\mathrm{CH}_{3} \mathrm{CN} / \mathrm{H}_{2} \mathrm{O}(0.2 \mathrm{~mL} / 0.8 \mathrm{~mL})$ as the solvent, & 66 \\
\hline & $5 \mathrm{~mol} \%\left[\operatorname{Ir}\left(\mathrm{dF}(\mathrm{ppy})_{2}(\mathrm{dtbbpy})\right] \mathrm{PF}_{6}\right.$ & \\
\hline \multirow[t]{2}{*}{12} & EtOAc $/ \mathrm{H}_{2} \mathrm{O}(0.5 \mathrm{~mL} / 0.5 \mathrm{~mL})$ as the solvent, & 76 \\
\hline & $5 \mathrm{~mol} \%\left[\operatorname{Ir}\left(\mathrm{dF}(\mathrm{ppy})_{2}(\mathrm{dtbbpy})\right] \mathrm{PF}_{6}\right.$ & \\
\hline
\end{tabular}


a. Product determined relative to 1,3,5-trimethoxybenzene as internal standard. b. Isolated yield. Reaction conditions: To an oven dried Schlenk tube with a magnetic stirring bar was added oxime ester (7, $59.3 \mathrm{mg}, 0$. $25 \mathrm{mmol}),{ }^{t} \mathrm{BuSSSS}^{\mathrm{t}} \mathrm{Bu}(\mathbf{1}, 24.2 \mathrm{mg}, 0.1 \mathrm{mmol})$, photocatalyst $(0.001 \mathrm{mmol})$ and $1.0 \mathrm{~mL}$ anhydrous solvent under nitrogen atmosphere and the resulting solution was degassed by three freeze-pump-thaw cycles (entry 5 was set up under air). After backfilling with nitrogen, the tube was placed in a photoreactor, stirred and irradiated with 4 LEDs for 12-14 hours. For entry 1-12, $33.6 \mathrm{mg}$ 1,3,5-trimethoxybenzene was added to the reaction tubes as the internal standard to determine the yield. The solvent was removed under reduced pressure and the crude residue was purified by column chromatography with hexanes as the eluent to afford the desired product 8 (35.6 mg, 87\% yield).

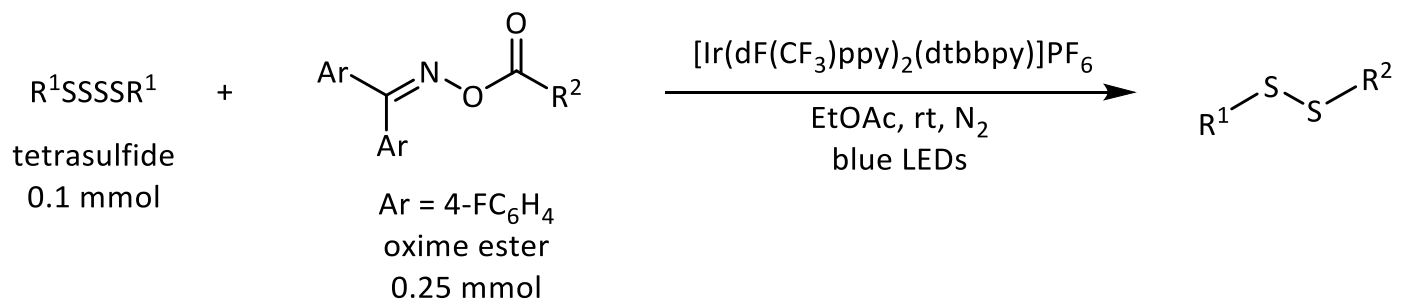

General synthetic procedure D: To an oven dried Schlenk tube with a magnetic stirring bar was added oxime ester $(0.25 \mathrm{mmol})$, tetrasulfide $(0.1 \mathrm{mmol}),\left[\operatorname{Ir}\left(\mathrm{dF}\left(\mathrm{CF}_{3}\right) \mathrm{ppy}\right)_{2}(\mathrm{dtbbpy})\right] \mathrm{PF}_{6}(1.2 \mathrm{mg}, 0.001 \mathrm{mmol})$ and $1.0 \mathrm{~mL}$ anhydrous EtOAc under nitrogen atmosphere and the resulting solution was degassed by three freeze-pumpthaw cycles. After backfilling with nitrogen, the tube was placed in a photoreactor, stirred and irradiated with 4 LEDs for 12-14 hours. The solvent was removed under reduced pressure and the crude residue was purified by column chromatography with hexanes as the eluent to afford the desired disulfide product.

\section{Control Experiments and Mechanistic Studies}

\subsection{Crossover experiment to demonstrate tetrasulfide lability}

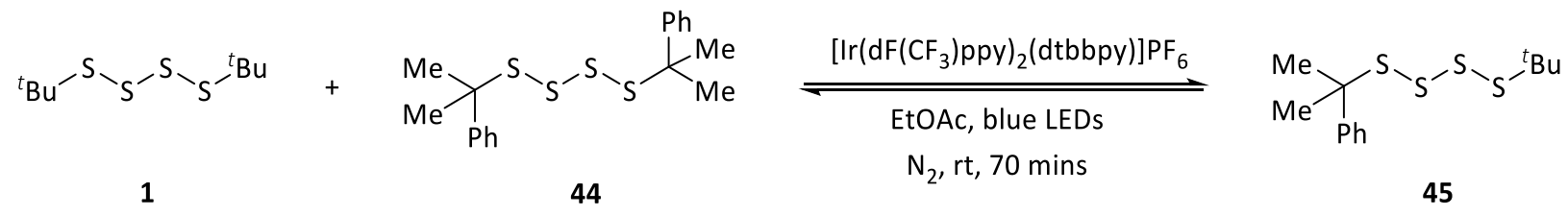

To an oven dried Schlenk tube with a magnetic stirring bar was added 1 ( $24.2 \mathrm{mg}, 0.1 \mathrm{mmol}), 44$ (36.7 mg, 0.1 $\mathrm{mmol})$ and $\left[\operatorname{Ir}\left(\mathrm{dF}\left(\mathrm{CF}_{3}\right) \mathrm{ppy}\right)_{2}(\mathrm{dtbbpy})\right] \mathrm{PF}_{6}(1.2 \mathrm{mg}, 0.001 \mathrm{mmol})$, followed by $1.0 \mathrm{~mL}$ anhydrous EtOAc. The resulting solution was degassed by three freeze-pump-thaw cycles. After backfilling with nitrogen, the tube was placed in a photoreactor, stirred and irradiated with 4 LEDs. Small volumes of the reaction mixture were withdrawn at 40 and 70 minutes and analyzed by ${ }^{1} \mathrm{H}$ NMR. The results are shown in Figure S1. The ratio 1:44:45 in the reaction mixture is roughly 1:1:2 after 70 mins. The remaining mixture was purified by column chromatography with hexanes as the eluent to obtain the NMR spectra of authentic 45. 


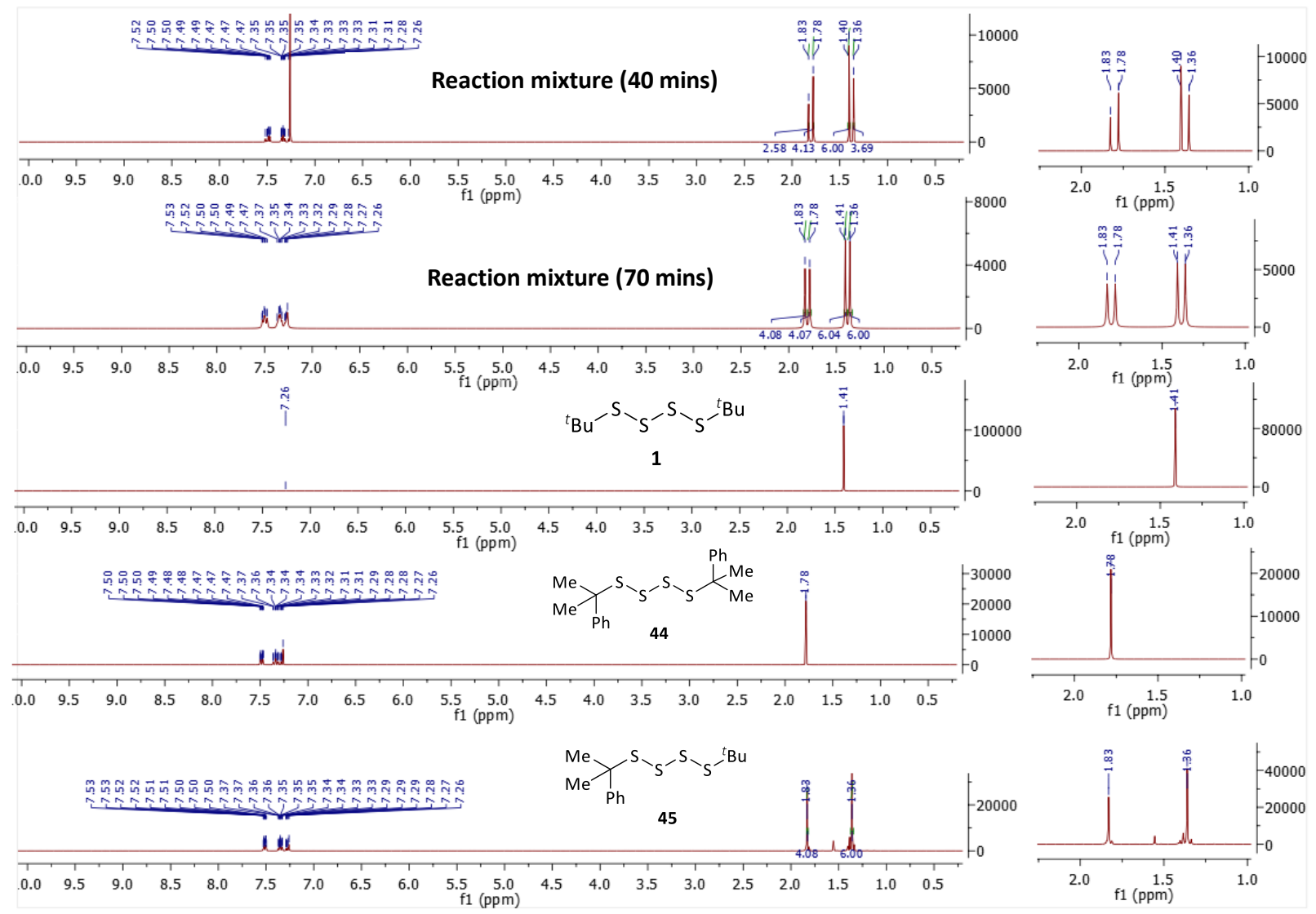

Figure S1. Crossover experiment in the presence of photocatalyst. ${ }^{1} \mathrm{H}$ NMR spectra of a 1:1 mixture of 1 and 44 after irradiation for 40 and 70 minutes showing a statistical $c a .1: 1: 2$ ratio of 1:44:45 in the latter. ${ }^{1} \mathrm{H}$ NMR spectra of authentic samples of $\mathbf{1 , 4 4 , 4 5}$ are shown for easy comparison.

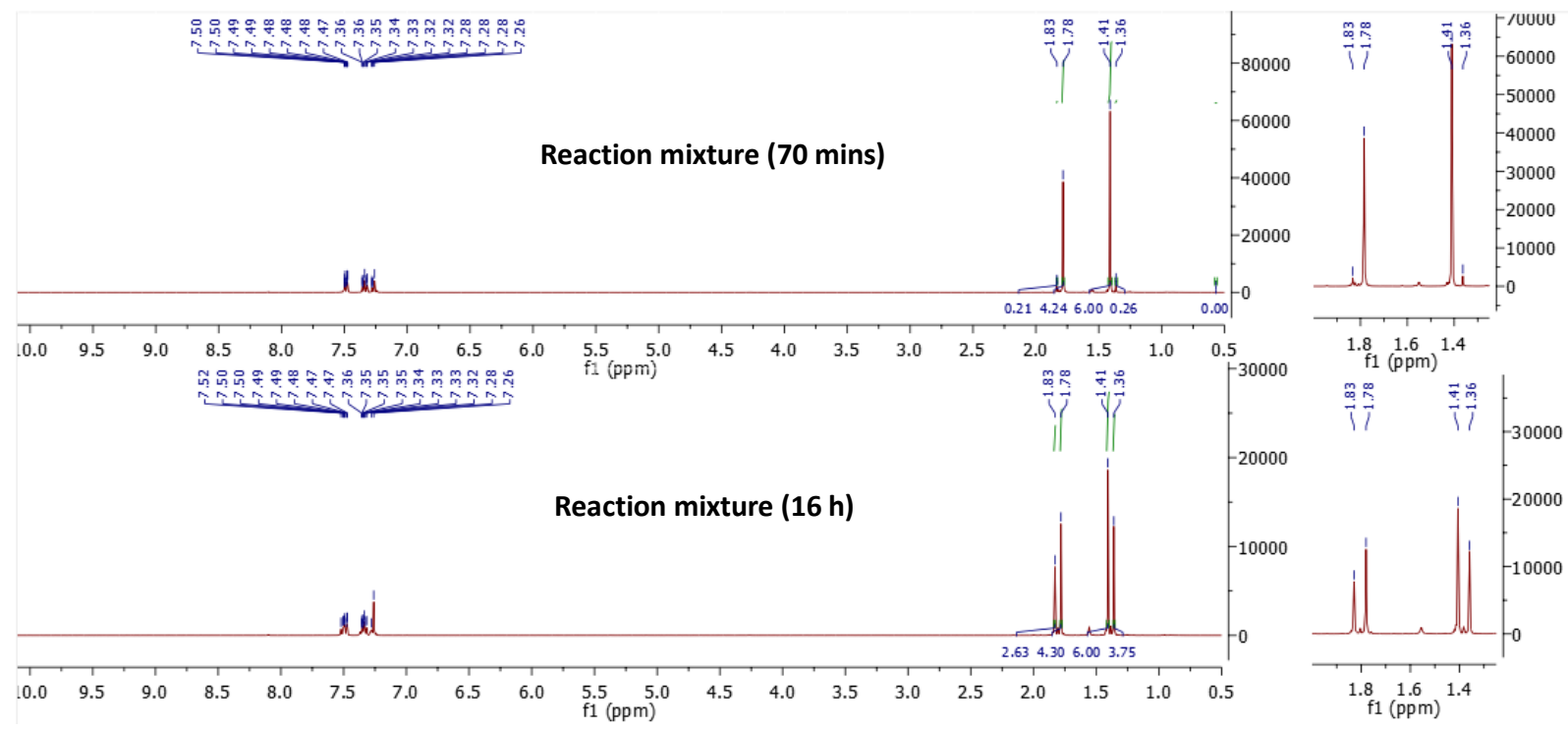

Figure S2. Crossover experiment in the absence of photocatalyst. ${ }^{1} \mathrm{H}$ NMR spectra of a 1:1 mixture of 1 and 44 after irradiation for 70 minutes and 16 hours showing substantially diminished crossover. 


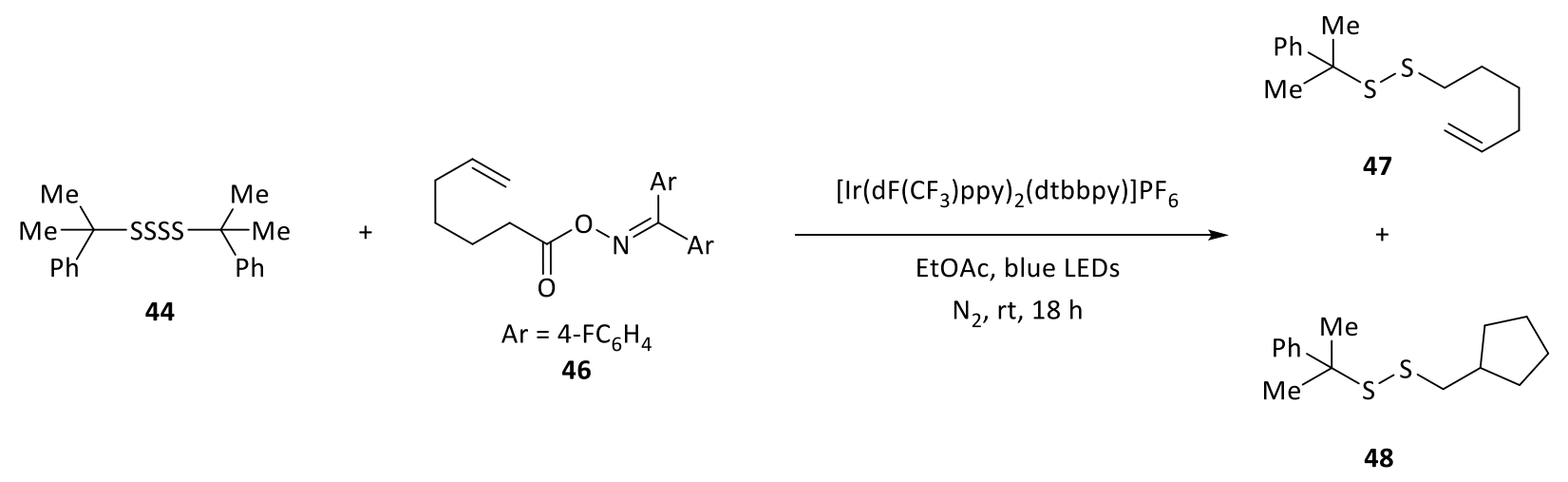

For each of three replicates, 5 samples were prepared containing $44(0.1 \mathrm{mmol}, 0.15 \mathrm{mmol}, 0.2 \mathrm{mmol}, 0.25$ mmol, $0.3 \mathrm{mmol}), 46(85.8 \mathrm{mg}, 0.25 \mathrm{mmol})$ and $\left[\operatorname{Ir}\left(\mathrm{dF}\left(\mathrm{CF}_{3}\right) \mathrm{ppy}\right)_{2}(\mathrm{dtbbpy})\right] \mathrm{PF} 6(1.2 \mathrm{mg}, 0.001 \mathrm{mmol})$ in $1 \mathrm{~mL}$ anhydrous EtOAc. The resulting solution was degassed by three freeze-pump-thaw cycles. After backfilling with nitrogen, the samples were irradiated for 18 hours. The solvent was then removed under reduced pressure and the crude residue was taken in $\mathrm{CDCl}_{3}$ and analyzed by ${ }^{1} \mathrm{H}$ NMR to determine the ratio of $\mathbf{4 7}$ to 48 . One of the reaction mixtures (reaction with $0.1 \mathrm{mmol} 44$ and $0.25 \mathrm{mmol} 46$ ) was purified by column chromatography with hexanes as the eluent to obtain spectra (and other characterization data) for authentic $\mathbf{4 7}$ and $\mathbf{4 8 .}$

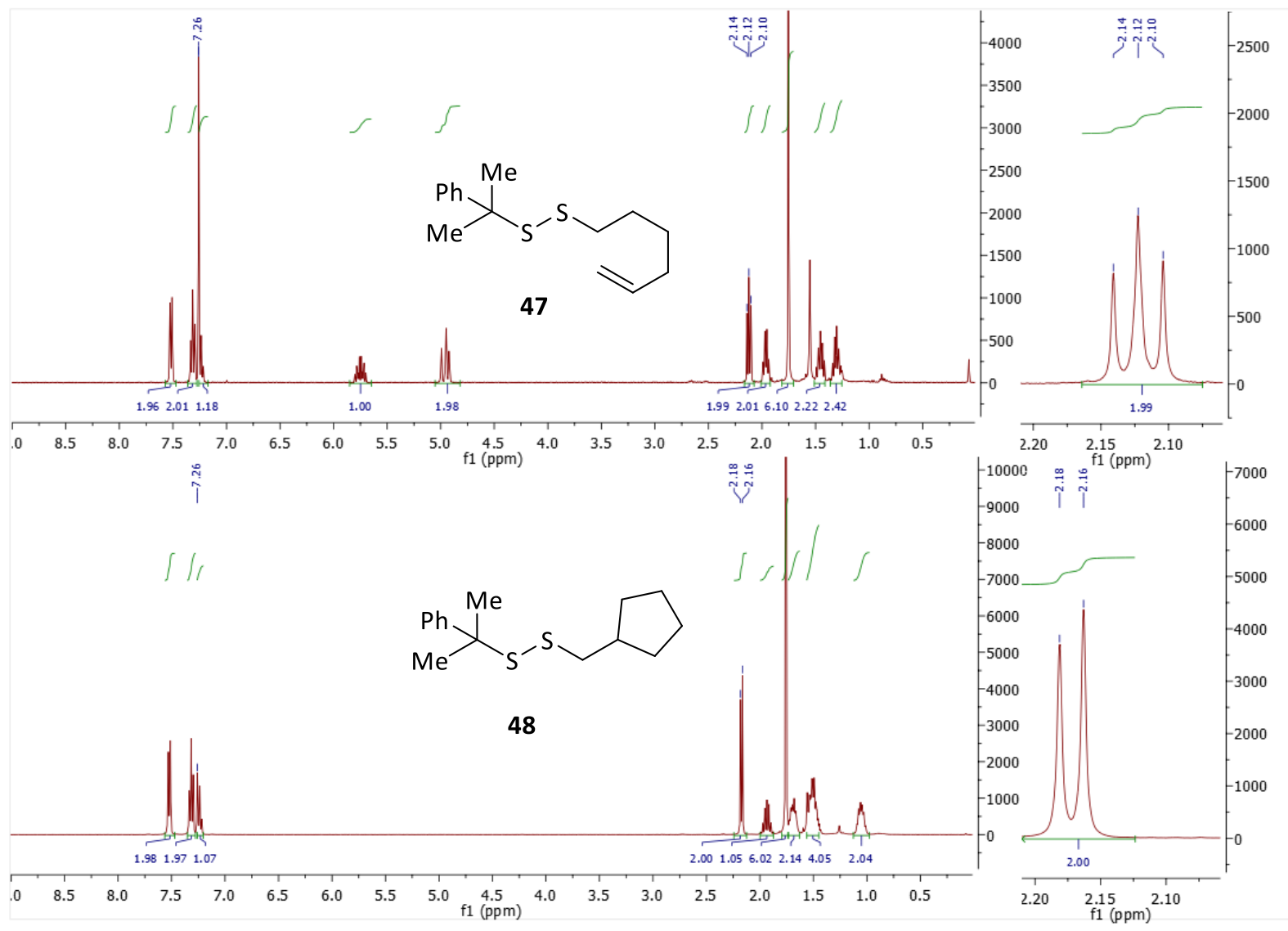

Figure S3. ${ }^{1} \mathrm{H}$ NMR spectra of $\mathbf{4 7}$ and 48. 

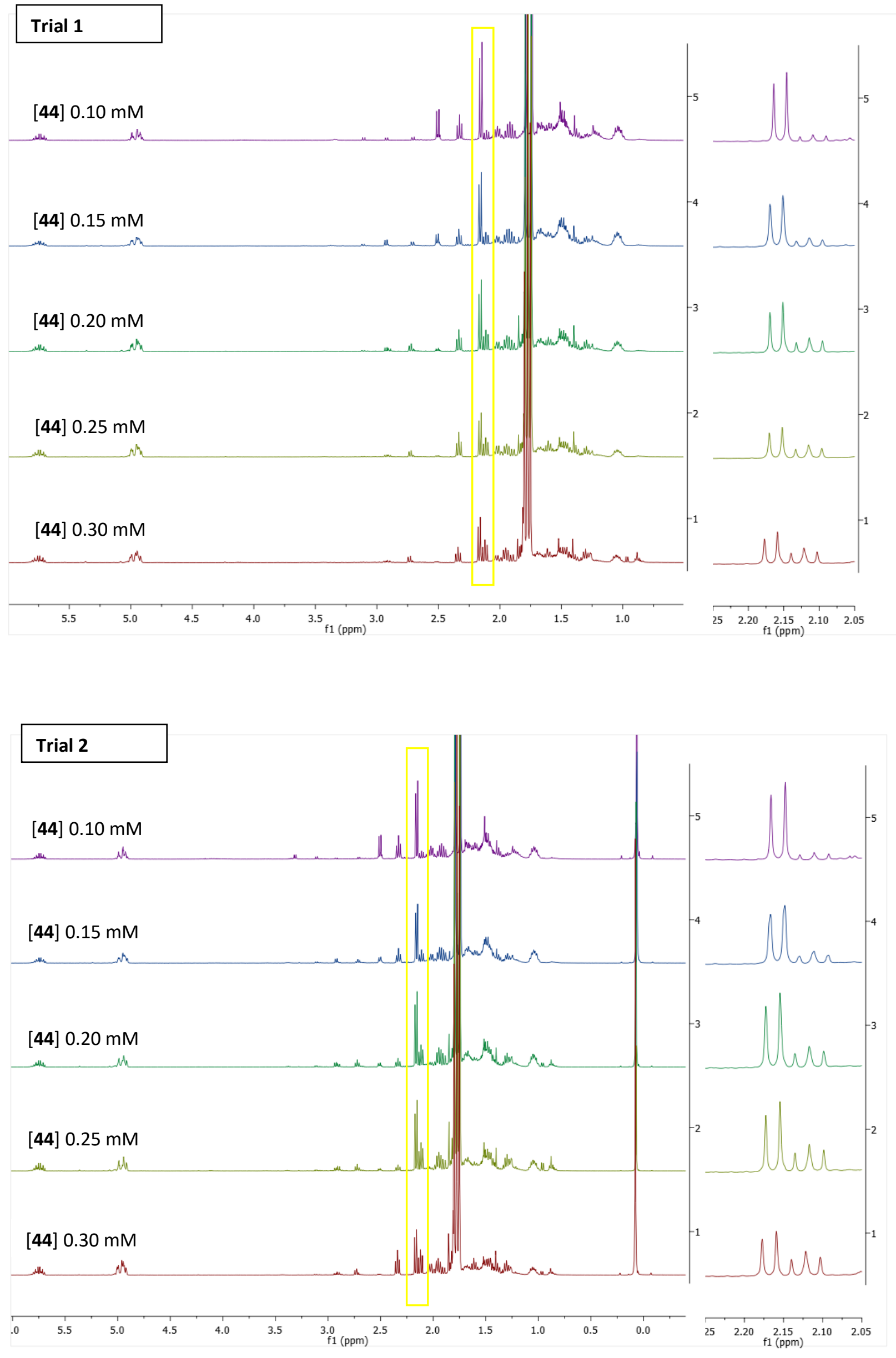


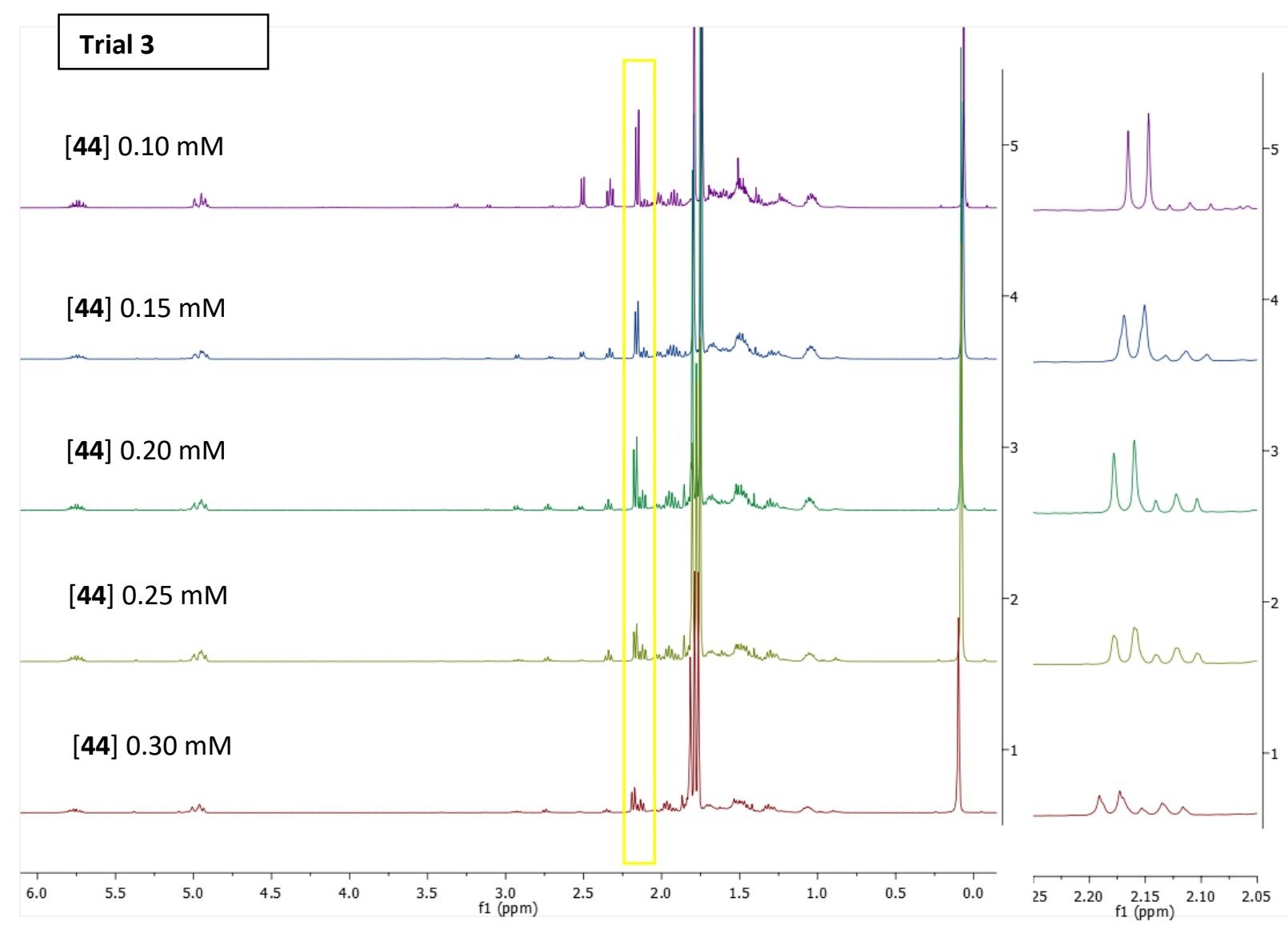

Figure S4. Crude ${ }^{1} \mathrm{H}$ NMR spectra of the reaction mixture obtained at different concentrations of $\mathbf{4 4}$ (from top to bottom, $0.1 \mathrm{mM}, 0.15 \mathrm{mM}, 0.2 \mathrm{mM}, 0.25 \mathrm{mM}, 0.3 \mathrm{mM}$ ).

Table S3. Product ratios 47:48 obtained from irradiation of $\mathbf{4 6}$ in the presence of increasing [44] as above.

\begin{tabular}{|c|c|c|c|c|c|}
\hline \multicolumn{2}{|c|}{ Trial 1 } & \multicolumn{2}{c|}{ Trial 2 } & \multicolumn{2}{c|}{ Trial 3 } \\
\hline$[\mathbf{4 4}] / \mathrm{mM}$ & {$[\mathbf{4 7}]:[\mathbf{4 8}]$} & {$[\mathbf{4 4}] / \mathrm{mM}$} & {$[\mathbf{4 7}]:[\mathbf{4 8}]$} & {$[\mathbf{4 4}] / \mathrm{mM}$} & {$[\mathbf{4 7}]:[\mathbf{4 8}]$} \\
\hline $\mathbf{0 . 1 0}$ & 0.22 & 0.10 & 0.20 & 0.10 & 0.21 \\
$\mathbf{0 . 1 5}$ & 0.35 & 0.15 & 0.32 & 0.15 & 0.31 \\
\hline $\mathbf{0 . 2 0}$ & 0.50 & 0.20 & 0.49 & 0.20 & 0.52 \\
\hline $\mathbf{0 . 2 5}$ & 0.70 & 0.25 & 0.72 & 0.25 & 0.71 \\
\hline $\mathbf{0 . 3 0}$ & 0.89 & 0.30 & 0.85 & 0.30 & 0.83 \\
\hline
\end{tabular}




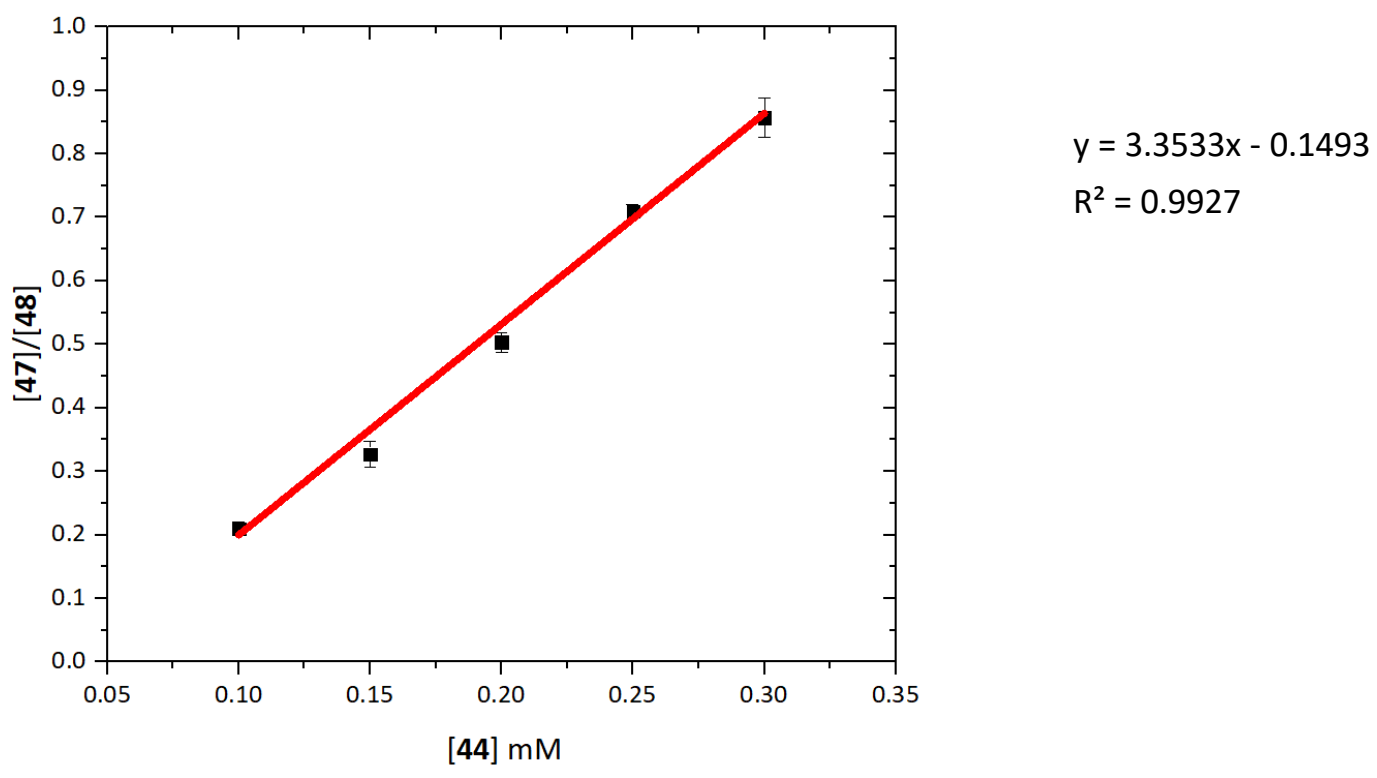

Figure S5. Ratio of unrearranged (47) and rearranged (48) products as a function of the concentration of tetrasulfide (44).

\subsection{Competition experiment between alkyl and acyl tetrasulfides}

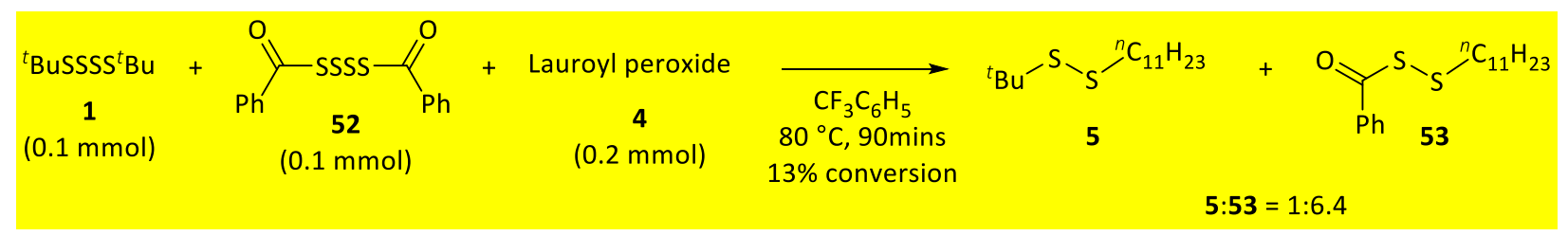

To an oven dried Schlenk tube with a magnetic stirring bar was added lauroyl peroxide $(4,79.7 \mathrm{mg}, 0.2 \mathrm{mmol})$, BzSSSSBz (52, $33.8 \mathrm{mg}, 0.1 \mathrm{mmol}),{ }^{t} B u S S S S{ }^{t} \mathrm{Bu}(1,24.2 \mathrm{mg}, 0.1 \mathrm{mmol})$ and $1.0 \mathrm{~mL} \mathrm{CF} \mathrm{C}_{6} \mathrm{H}_{5}$ under nitrogen atmosphere and the resulting solution was degassed by three freeze-pump-thaw cycles. After back filling with nitrogen, the tube was heated to $80{ }^{\circ} \mathrm{C}$ with stirring. A small amount of the crude residue was taken out at different time points and analyzed by ${ }^{1} \mathrm{H}$ NMR to determine the product ratios. 


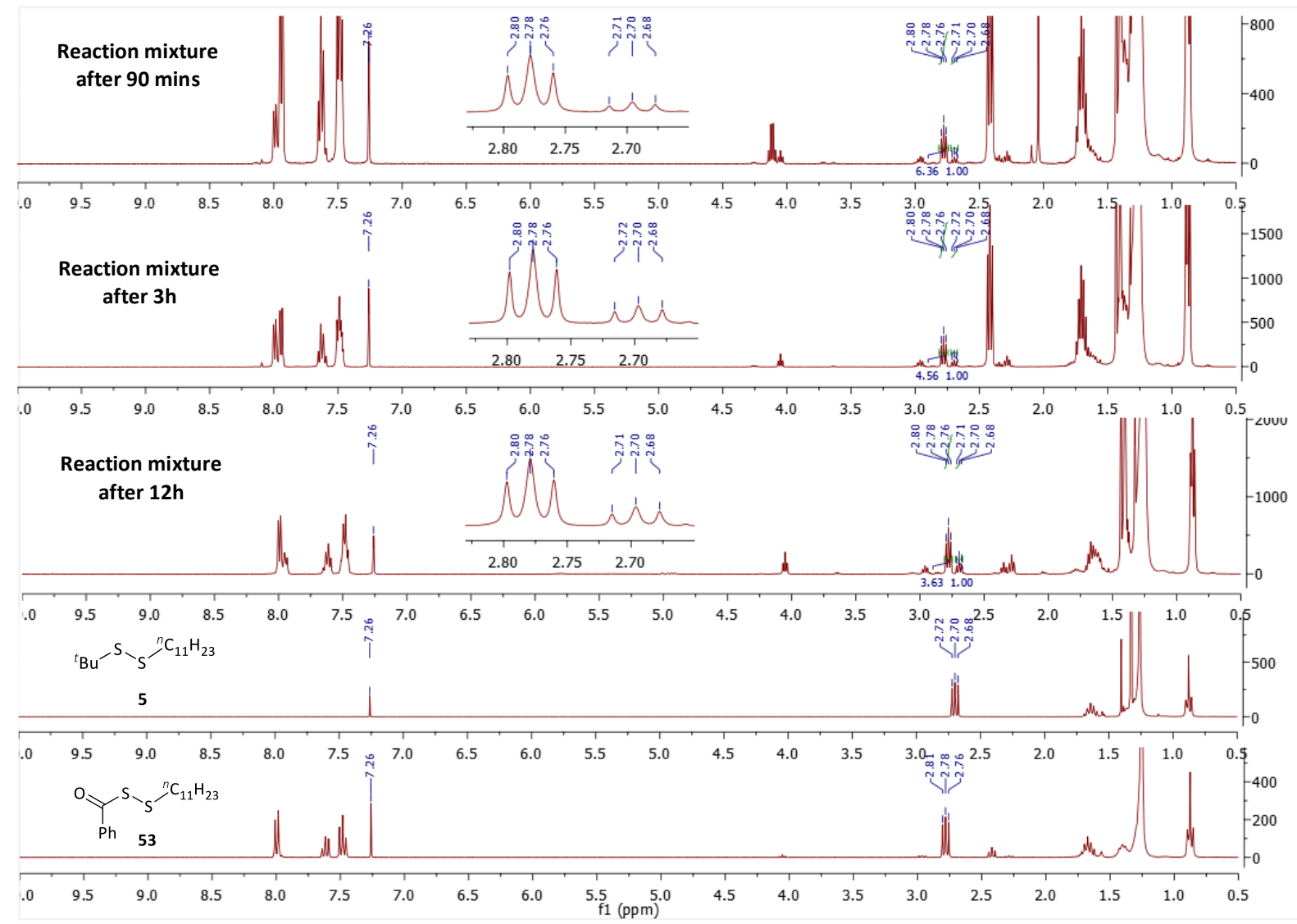

Figure S6. Product distribution for competing radical substitution on di-tert-butyl tetrasulfide and dibenzoyl tetrasulfide at $90 \mathrm{~min}, 3 \mathrm{~h}$ and $12 \mathrm{~h}$, which correspond to ca. $13 \%$, ca. $20 \%$ and effectively complete conversion based on loss of lauroyl peroxide.

\section{Additional Synthetic Transformations}

4.1 Use of an organic photosensitizer instead of iridium-based photosensitizer as the catalyst

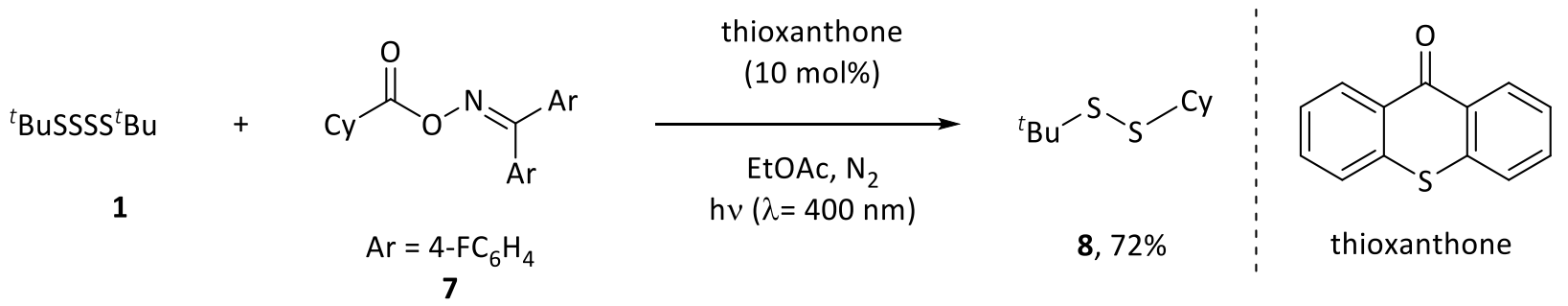

To an oven dried Schlenk tube with a magnetic stirring bar was added oxime ester $7(85.8 \mathrm{mg}, 0.25 \mathrm{mmol})$, ${ }^{t}$ BuSSSS ${ }^{t} \mathrm{Bu}(1,24.2 \mathrm{mg}, 0.1 \mathrm{mmol})$, thioxanthone $(2.1 \mathrm{mg}, 0.01 \mathrm{mmol})$ and $1.0 \mathrm{~mL}$ anhydrous EtOAc under nitrogen atmosphere and the resulting solution was degassed by three freeze-pump-thaw cycles. After backfilling with nitrogen, the tube was placed in a photoreactor, stirred and irradiated with LEDs $(\lambda=400 \mathrm{~nm})$ 
for 12-14 hours. The solvent was removed under reduced pressure and the crude residue was purified by column chromatography with hexanes as the eluent to afford the desired product 8 ( $29.4 \mathrm{mg}, 72 \%$ yield).

\subsection{Larger scale reaction to produce disulfide 17}

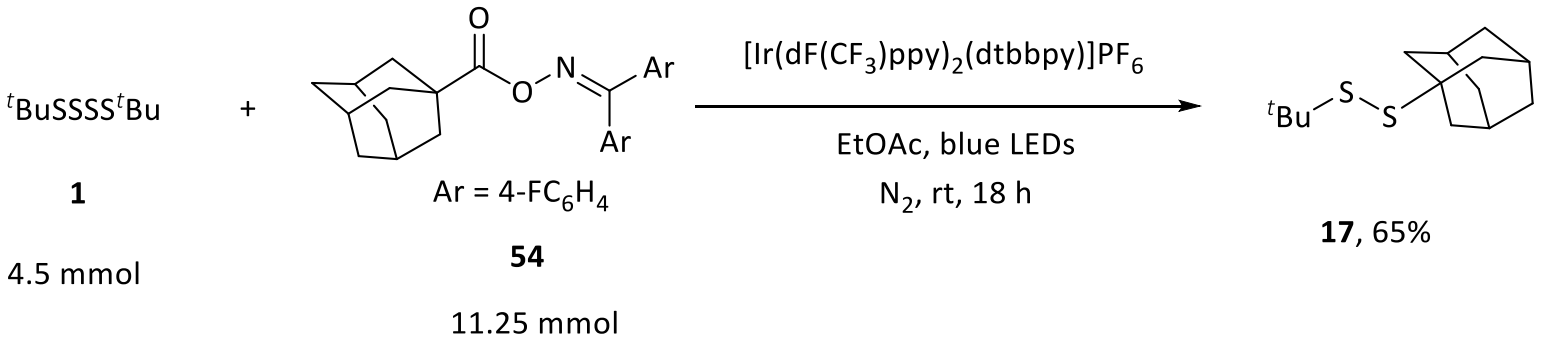

Follow the general procedure $D$, to an oven dried Schlenk tube with a magnetic stirring bar was added oxime ester 54 (4.45 g, $11.25 \mathrm{mmol})$, tetrasulfide 1 (1.09 g, $4.5 \mathrm{mmol})$, [Ir(dF(CF $)$ ppy $\left._{2}(\mathrm{dtbbpy})\right] \mathrm{PF}_{6}(55 \mathrm{mg}, 0.045$ $\mathrm{mmol}$ ) and $45 \mathrm{~mL}$ anhydrous EtOAc under nitrogen atmosphere and the resulting solution was degassed by three freeze-pump-thaw cycles. After backfilling with nitrogen, the tube was placed in a photoreactor, stirred and irradiated with 4 LEDs for 12-14 hours. The solvent was removed under reduced pressure and the crude residue was purified by column chromatography with hexanes as the eluent to afford $\mathbf{1 7}$ (1.50 g, 65\% yield).

\subsection{Synthesis of acylpersulfides from Ac-/Bz- tetrasulfide}

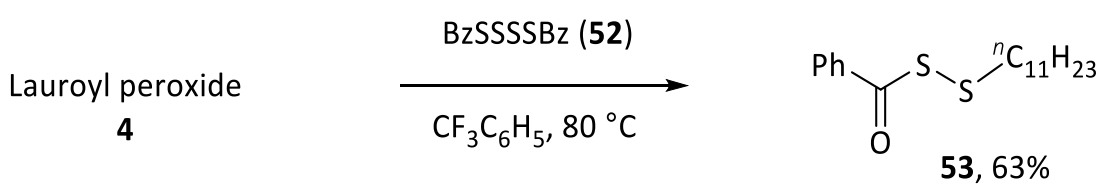

To an oven dried Schlenk tube with a magnetic stirring bar was added lauroyl peroxide $(4,79.7 \mathrm{mg}, 0.2 \mathrm{mmol})$, $\operatorname{BzSSSSBz}(52,33.8 \mathrm{mg}, 0.1 \mathrm{mmol})$ and $1.0 \mathrm{~mL} \mathrm{CF}_{3} \mathrm{C}_{6} \mathrm{H}_{5}$ under nitrogen atmosphere and the resulting solution was degassed by three freeze-pump-thaw cycles. After back filling with nitrogen, the tube was heated to $80^{\circ} \mathrm{C}$ with stirring for 12-14 hours. The solvent was removed under reduced pressure and the crude residue was purified by column chromatography with hexanes as the eluent to afford the desired product 53 (40.9 mg, 63\% yield).

Lauroyl peroxide

4
AcSSSSAc (51)

$$
\mathrm{CF}_{3} \mathrm{C}_{6} \mathrm{H}_{5}, 80^{\circ} \mathrm{C}
$$

$$
\mathrm{Ac}^{-S} \mathrm{~S}_{S^{\prime}}{ }^{n} \mathrm{C}_{11} \mathrm{H}_{23}
$$

S1, $56 \%$

To an oven dried Schlenk tube with a magnetic stirring bar was added lauroyl peroxide $(4,79.7 \mathrm{mg}, 0.2 \mathrm{mmol})$, AcSSSSAc (51, $21.4 \mathrm{mg}, 0.1 \mathrm{mmol})$ and $1.0 \mathrm{~mL} \mathrm{CF}{ }_{3} \mathrm{C}_{6} \mathrm{H}_{5}$ under nitrogen atmosphere and the resulting solution was degassed by three freeze-pump-thaw cycles. After back filling with nitrogen, the tube was heated to $80^{\circ} \mathrm{C}$ with stirring for 12-14 hours. The solvent was removed under reduced pressure and the crude residue was 
purified by column chromatography with hexanes as the eluent to afford the desired product S1 $(29.4 \mathrm{mg}, 56 \%$ yield).

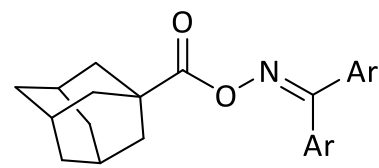

$\mathrm{Ar}=4-\mathrm{FC}_{6} \mathrm{H}_{4}$
AcSSSSAc (51)

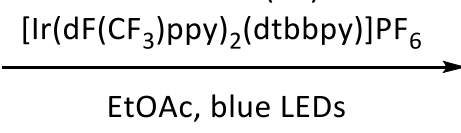

EtOAc, blue LEDs

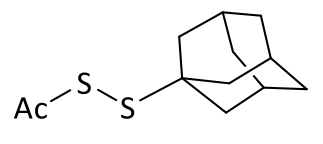

$55,48 \%$

54

To an oven dried Schlenk tube with a magnetic stirring bar were added oxime ester $\mathbf{5 4}(98.8 \mathrm{mg}, 0.25 \mathrm{mmol})$, AcSSSSAc $(51,21.4 \mathrm{mg}, 0.1 \mathrm{mmol}),\left[\operatorname{Ir}\left(\mathrm{dF}\left(\mathrm{CF}_{3}\right) \mathrm{ppy}\right)_{2}(\mathrm{dtbbpy})\right] \mathrm{PF} 6(1.2 \mathrm{mg}, 0.001 \mathrm{mmol})$ followed by $1 \mathrm{~mL}$ anhydrous EtOAc under a nitrogen atmosphere and the resulting solution was degassed by three freeze-pumpthaw. After backfilling with nitrogen, the tube was placed in a photoreactor, stirred and irradiated with 4 LEDs for 12-14 hours. The solvent was removed under reduced pressure and the crude residue was purified by column chromatography with hexanes as the eluent to afford the desired product 55 ( $23.3 \mathrm{mg}, 48 \%$ yield).<smiles>CC(C)(C)C(=O)ON=C(Br)Br</smiles>

$\mathrm{Ar}=4-\mathrm{FC}_{6} \mathrm{H}_{4}$<smiles>O=C(SC(=O)c1ccccc1)c1ccccc1</smiles>

(52)

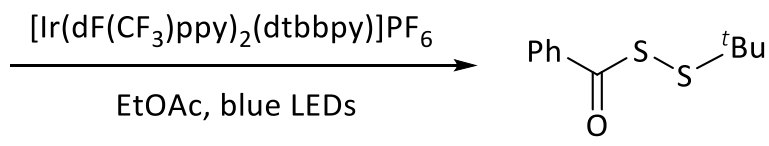

S3

S2

Following the procedure above, to an oven dried Schlenk tube with a magnetic stirring bar was added oxime ester S2 (79.3 mg, 0. 25 mmol), BzSSSSBz (52, $33.8 \mathrm{mg}, 0.1 \mathrm{mmol}),\left[\operatorname{Ir}\left(\mathrm{dF}\left(\mathrm{CF}_{3}\right)\right.\right.$ ppy) $\left.2(\mathrm{dtbbpy})\right] \mathrm{FF}_{6}(1.2 \mathrm{mg}, 0.001$ $\mathrm{mmol}$ ) followed by $1 \mathrm{~mL}$ anhydrous EtOAc under nitrogen atmosphere and the resulting solution was degassed by three freeze-pump-thaw cycles. After backfilling with nitrogen, the tube was placed in a photoreactor, stirred and irradiated with 4 LEDs for 12-14 hours. The solvent was removed under reduced pressure and the crude residue was purified by column chromatography with hexanes as the eluent to afford the desired product S3 (18.6 mg, 41\% yield).

\subsection{Three-component carbodisulfuration of alkene}

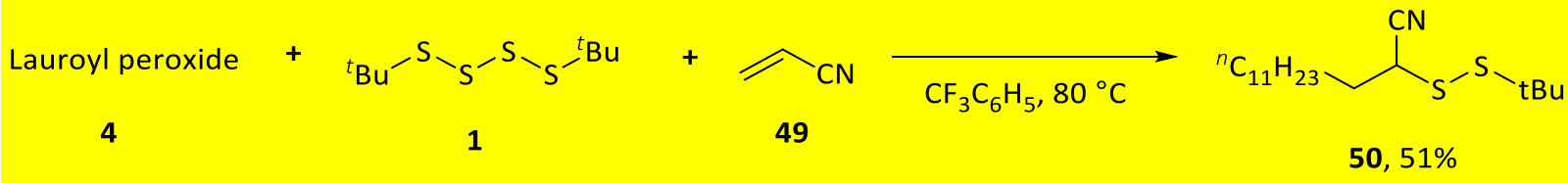

To an oven dried Schlenk tube with a magnetic stirring bar was added lauroyl peroxide $(4,59.8 \mathrm{mg}, 0.15 \mathrm{mmol})$, ${ }^{t} \mathrm{BuSSSS}^{t} \mathrm{Bu}(1,24.2 \mathrm{mg}, 0.1 \mathrm{mmol})$, acrylonitrile $(49,8.6 \mathrm{mg}, 0.1 \mathrm{mmol})$ and $2.0 \mathrm{~mL} \mathrm{CF}_{3} \mathrm{C}_{6} \mathrm{H}_{5}$ under nitrogen atmosphere and the resulting solution was degassed by three freeze-pump-thaw cycles. After back filling with 
nitrogen, the tube was heated to $80^{\circ} \mathrm{C}$ for 12 hours. The solvent was removed under reduced pressure and the crude residue was purified by column chromatography with hexanes as the eluent to afford the desired product 50 (16.8 mg, 51\% yield).

\section{Characterization Data}<smiles>CC(C)(C)CSSC(C)(C)C#N</smiles>

3

2-(tert-butyldisulfanyl)-2-methylpropanenitrile (3): Following the synthetic procedure A, $\mathbf{3}$ was obtained as a colorless oil (64.4 mg, 85\% yield). ${ }^{1} \mathrm{H}$ NMR (400 MHz, Chloroform-d) $\delta 1.66(\mathrm{~s}, 6 \mathrm{H}), 1.40(\mathrm{~s}, 9 \mathrm{H}) .{ }^{13} \mathrm{C}$ NMR $(101$ $\mathrm{MHz}, \mathrm{CDCl}_{3}$ ) $\delta 123.2,49.0,40.3,30.9,27.5$. HRMS (EI) m/z calcd for $\mathrm{C}_{8} \mathrm{H}_{15} \mathrm{NS}_{2}\left[\mathrm{M}^{+}\right]$: 189.0646, found 189.0642.

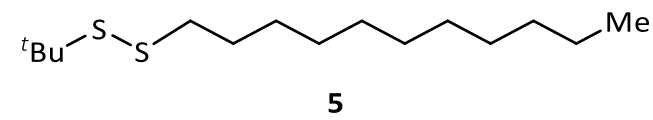

1-(tert-butyl)-2-undecyldisulfane (5): Following the synthetic procedure B, $\mathbf{5}$ was obtained as a colorless oil (51.9 mg, 94\% yield). ${ }^{1} \mathrm{H}$ NMR (300 MHz, Chloroform-d) $\delta 2.70(\mathrm{t}, J=6.0 \mathrm{~Hz}, 2 \mathrm{H}), 1.69-1.59(\mathrm{~m}, 2 \mathrm{H}), 1.39-1.33(\mathrm{~m}, J=$ 2H), $1.33(\mathrm{~s}, 9 \mathrm{H}), 1.32-1.22(\mathrm{~m}, 12 \mathrm{H}), 0.88(\mathrm{t}, J=6.0 \mathrm{~Hz}, 2 \mathrm{H}) .{ }^{13} \mathrm{C}$ NMR (75 MHz, Chloroform-d) $\delta$ 47.8, 41.1, 32.1, 30.4, 30.1, 29.8, 29.7, 29.5, 29.4, 28.8, 22.8, 14.3. HRMS (EI) m/z calcd for $\mathrm{C}_{15} \mathrm{H}_{32} \mathrm{~S}_{2}\left[\mathrm{M}^{+}\right]$: 276.1945, found 276.1948 .

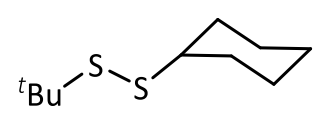

1-(tert-butyl)-2-cyclohexyldisulfane (8): Following the synthetic procedure $\mathbf{C}$ (or D), $\mathbf{8}$ was obtained as a colorless oil (C: $36.8 \mathrm{mg}, 90 \%$ yield; D: $35.6 \mathrm{mg}, 87 \%$ yield). ${ }^{1} \mathrm{H}$ NMR (400 MHz, Chloroform-d) $\delta 2.72-2.65$ (m, 1H), $2.10-$ $2.05(\mathrm{~m}, 2 \mathrm{H}), 1.81-1.76(\mathrm{~m}, 2 \mathrm{H}), 1.62-1.59(\mathrm{~m}, 1 \mathrm{H}), 1.32(\mathrm{~s}, 9 \mathrm{H}), 1.31-1.22(\mathrm{~m}, 5 \mathrm{H}) .{ }^{13} \mathrm{C} \mathrm{NMR}(101 \mathrm{MHz}$, Chloroform-d) $\delta 50.4,47.3,33.0,30.0,26.1,25.8$. HRMS (EI) $\mathrm{m} / \mathrm{z}$ calcd for $\mathrm{C}_{10} \mathrm{H}_{20} \mathrm{~S}_{2}\left[\mathrm{M}^{+}\right]: 204.1006$, found 204.1005.

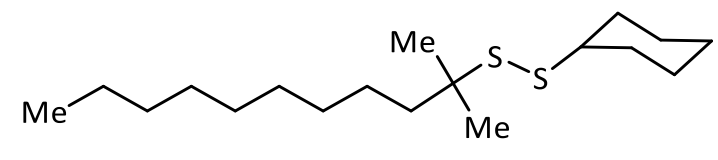

9

1-cyclohexyl-2-(2-methylundecan-2-yl)disulfane (9): Following the synthetic procedure C (or D), 9 was obtained as a colorless oil (C: $50.6 \mathrm{mg}, 80 \%$ yield; D: $48.8 \mathrm{mg}, 77 \%$ yield). ${ }^{1} \mathrm{H}$ NMR (400 MHz, Chloroform-d) $\delta 2.65$ (ddq, J $=10.5,7.5,3.8 \mathrm{~Hz}, 1 \mathrm{H}), 2.10-2.05(\mathrm{~m}, 2 \mathrm{H}), 1.80-1.76(\mathrm{~m}, 2 \mathrm{H}), 1.62-1.59(\mathrm{~m}, 1 \mathrm{H}), 1.53-1.51(\mathrm{~m}, 2 \mathrm{H}), 1.37-1.22$ 
(m, 25H), 0.88 (t, $J=6.7 \mathrm{~Hz}, 3 \mathrm{H}) .{ }^{13} \mathrm{C}$ NMR $(101 \mathrm{MHz}$, Chloroform-d) $\delta 50.8,50.3,41.8,33.1,32.1,30.3,29.8,29.8$, 29.5, 27.9, 26.2, 25.9, 25.0, 22.8, 14.3. HRMS (EI) m/z calcd for $\mathrm{C}_{18} \mathrm{H}_{36} \mathrm{~S}_{2}\left[\mathrm{M}^{+}\right]$: 316.2258, found 316.2262 .

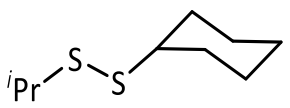

10

1-cyclohexyl-2-isopropyldisulfane (10): Following the synthetic procedure D, 10 was obtained as a colorless oil (34.3 mg, 90\% yield). ${ }^{1} \mathrm{H}$ NMR (400 MHz, Chloroform-d) $\delta 2.9-2.92(\mathrm{~m}, 1 \mathrm{H}), 2.72-2.66(\mathrm{~m}, 1 \mathrm{H}), 2.06-2.02(\mathrm{~m}$, $2 \mathrm{H}), 1.80-1.76(\mathrm{~m}, 2 \mathrm{H}), 1.63-1.60(\mathrm{~m}, 1 \mathrm{H}), 1.35-1.31(\mathrm{~m}, 3 \mathrm{H}), 1.30(\mathrm{~s}, 3 \mathrm{H}), 1.28(\mathrm{~d}, J=1.9 \mathrm{~Hz}, 3 \mathrm{H}), 1.27-1.20(\mathrm{~m}$, 2H). ${ }^{13} \mathrm{C}$ NMR (101 MHz, Chloroform-d) $\delta 50.1,41.6,33.0,26.2,25.9,22.7$. HRMS (EI) m/z calcd for $\mathrm{C}_{9} \mathrm{H}_{18} \mathrm{~S}_{2}\left[\mathrm{M}^{+}\right]$: 190.0850, found 190.0853 .

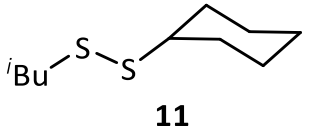

1-cyclohexyl-2-isobutyldisulfane (11): Following the synthetic procedure D, 11 was obtained as a colorless oil (38.0 mg, 93\% yield). ${ }^{1} \mathrm{H}$ NMR (400 MHz, Chloroform-d) $\delta 2.74-2.68(\mathrm{~m}, 1 \mathrm{H}), 2.59(\mathrm{~d}, \mathrm{~J}=6.9 \mathrm{~Hz}, 2 \mathrm{H}), 2.04-2.01$ $(\mathrm{m}, 2 \mathrm{H}), 1.92(\mathrm{dt}, J=13.5,6.7 \mathrm{~Hz}, 1 \mathrm{H}), 1.80-1.77(\mathrm{~m}, 2 \mathrm{H}), 1.63-1.60(\mathrm{~m}, 1 \mathrm{H}), 1.37-1.23(\mathrm{~m}, 5 \mathrm{H}), 0.99(\mathrm{~d}, J=6.7$

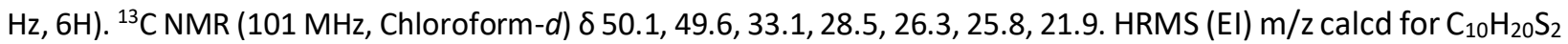
$\left[\mathrm{M}^{+}\right]:$204.1006, found 204.1005.

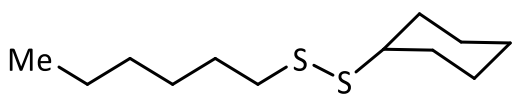

12

1-cyclohexyl-2-hexyldisulfane (12): Following the synthetic procedure C (or D), 12 was obtained as a colorless oil (C: $42.3 \mathrm{mg}, 91 \%$ yield; D: $41.4 \mathrm{mg}, 89 \%$ yield). ${ }^{1} \mathrm{H}$ NMR (400 MHz, Chloroform-d) $\delta 2.72-2.66(\mathrm{~m}, 3 \mathrm{H}), 2.05-2.01(\mathrm{~m}$, $2 \mathrm{H}), 1.81-1.77(\mathrm{~m}, 2 \mathrm{H}), 1.67-1.62(\mathrm{~m}, 3 \mathrm{H}), 1.40-1.24(\mathrm{~m}, 11 \mathrm{H}), 0.89(\mathrm{t}, \mathrm{J}=6.8 \mathrm{~Hz}, 3 \mathrm{H}) .{ }^{13} \mathrm{C} \mathrm{NMR}(101 \mathrm{MHz}$, Chloroform-d) $\delta 50.1,40.5,39.4,33.0,31.6,29.4,28.4,26.3,22.7,14.2$. HRMS (EI) m/z calcd for $\mathrm{C}_{12} \mathrm{H}_{24} \mathrm{~S}_{2}\left[\mathrm{M}^{+}\right]$: 232.1319, found 232.1315.

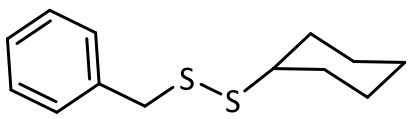

13

1-benzyl-2-cyclohexyldisulfane (13): Following the synthetic procedure D, 13 was obtained as a colorless oil (41.9 $\mathrm{mg}, 88 \%$ yield). ${ }^{1} \mathrm{H}$ NMR (400 MHz, Chloroform-d) $\delta 7.33-7.26(\mathrm{~m}, 5 \mathrm{H}), 3.89(\mathrm{~s}, 2 \mathrm{H}), 2.42-2.35$ (m, $\left.1 \mathrm{H}\right), 1.97-1.92$ (m, 2H), $1.76-1.72(\mathrm{~m}, 2 \mathrm{H}), 1.59-1.57(\mathrm{~m}, 1 \mathrm{H}), 1.29-1.15(\mathrm{~m}, 5 \mathrm{H}) .{ }^{13} \mathrm{C}$ NMR (101 MHz, Chloroform-d) $\delta 137.8$, 129.4, 128.6, 127.5, 49.4, 44.8, 32.9, 26.2, 25.7. HRMS (EI) m/z calcd for $\mathrm{C}_{13} \mathrm{H}_{18} \mathrm{~S}_{2}\left[\mathrm{M}^{+}\right]$: 238.0850, found 238.0849. 


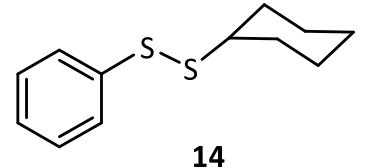

1-cyclohexyl-2-phenyldisulfane (14): Following the synthetic procedure D, 14 was obtained as a light brown oil (31.9 mg, 71\% yield). ${ }^{1} \mathrm{H}$ NMR (300 MHz, Chloroform-d) $\delta 7.56-7.52(\mathrm{~m}, 2 \mathrm{H}), 7.34-7.26(\mathrm{~m}, 2 \mathrm{H}), 7.22-7.17$ (m, $1 \mathrm{H}), 2.81(\mathrm{tt}, J=10.7,3.7 \mathrm{~Hz}, 1 \mathrm{H}), 2.05-1.99(\mathrm{~m}, 2 \mathrm{H}), 1.79-1.73(\mathrm{~m}, 2 \mathrm{H}), 1.62-1.57(\mathrm{~m}, 1 \mathrm{H}), 1.40-1.21(\mathrm{~m}, 5 \mathrm{H})$. ${ }^{13} \mathrm{C}$ NMR (101 MHz, Chloroform-d) $\delta$ 138.5, 128.8, 126.8, 126.3, 49.9, 32.6, 26.0, 25.6. HRMS (EI) m/z calcd for $\mathrm{C}_{12} \mathrm{H}_{16} \mathrm{~S}_{2}\left[\mathrm{M}^{+}\right]: 224.0693$, found 224.0691.

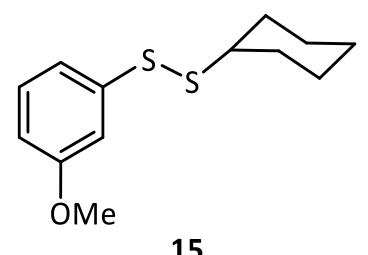

1-cyclohexyl-2-(3-methoxyphenyl)disulfane (15): Following the synthetic procedure C (or D), 15 was obtained as a light brown oil (C: $36.6 \mathrm{mg}, 72 \%$ yield; D: $38.7 \mathrm{mg}, 76 \%$ yield). ${ }^{1} \mathrm{H}$ NMR (400 MHz, Chloroform-d) $\delta 7.21$ (t, J=8.0 Hz, $1 \mathrm{H}), 7.13-7.08(\mathrm{~m}, 2 \mathrm{H}), 6.73$ (ddd, $J=8.1,2.5,1.0 \mathrm{~Hz}, 1 \mathrm{H}), 3.82(\mathrm{~s}, 3 \mathrm{H}), 2.83-2.78(\mathrm{~m}, 1 \mathrm{H}), 2.05-2.00(\mathrm{~m}, 2 \mathrm{H}), 1.79$ - $1.74(\mathrm{~m}, 2 \mathrm{H}), 1.62-1.56(\mathrm{~m}, 1 \mathrm{H}), 1.40-1.21(\mathrm{~m}, 5 \mathrm{H}) .{ }^{13} \mathrm{C}$ NMR (101 MHz, Chloroform-d) $\delta$ 160.1, 139.9, 129.8, 118.9, 112.3, 111.9, 55.5, 50.1, 32.8, 26.2, 25.7. HRMS (EI) $\mathrm{m} / \mathrm{z}$ calcd for $\mathrm{C}_{13} \mathrm{H}_{18} \mathrm{OS}_{2}\left[\mathrm{M}^{+}\right]: 254.0799$, found 254.0798 .

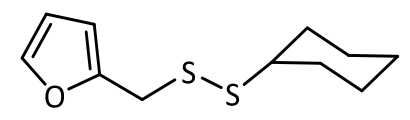

16

2-((cyclohexyldisulfanyl)methyl)furan (16): Following the synthetic procedure $\mathbf{D}, \mathbf{1 6}$ was obtained as a colorless oil (37.9 mg, 83\% yield). ${ }^{1} \mathrm{H}$ NMR (400 MHz, Chloroform-d) $\delta 7.38(\mathrm{~s}, 1 \mathrm{H}), 6.32(\mathrm{~m}, 1 \mathrm{H}), 6.25(\mathrm{~d}, J=3.2 \mathrm{~Hz}, 1 \mathrm{H}$ ), 3.89 $(\mathrm{s}, 2 \mathrm{H}), 2.44-2.38(\mathrm{~m}, 1 \mathrm{H}), 1.98-1.93(\mathrm{~m}, 2 \mathrm{H}), 1.77-1.73(\mathrm{~m}, 2 \mathrm{H}), 1.61-1.58(\mathrm{~m}, 1 \mathrm{H}), 1.29-1.19(\mathrm{~m}, 5 \mathrm{H}) .{ }^{13} \mathrm{C}$ NMR (101 MHz, Chloroform-d) $\delta 150.8,142.5,110.8,108.8,49.6,37.0,32.9,26.3,25.8$. HRMS (EI) m/z calcd for $\mathrm{C}_{11} \mathrm{H}_{16} \mathrm{OS}_{2}\left[\mathrm{M}^{+}\right]$: 228.0643, found 228.0645 .

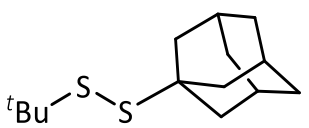

17

1-((1S,3R,5S)-adamantan-1-yl)-2-(tert-butyl)disulfane (17): Following the synthetic procedure C (or D), 17 was obtained as a colorless oil (C: $35.9 \mathrm{mg}, 70 \%$ yield; D: $34.4 \mathrm{mg}, 67 \%$ yield). ${ }^{1} \mathrm{H}$ NMR (400 MHz, Chloroform-d) $\delta 2.06$ (s, $3 \mathrm{H}), 1.82(\mathrm{~d}, J=3.1 \mathrm{~Hz}, 6 \mathrm{H}), 1.70-1.62(\mathrm{~m}, 6 \mathrm{H}), 1.30(\mathrm{~s}, 9 \mathrm{H}) .{ }^{13} \mathrm{C}$ NMR $(75 \mathrm{MHz}$, Chloroform-d) $\delta$ 47.9, 45.9, 43.3, 36.3, 30.7, 30.2. HRMS (EI) $\mathrm{m} / \mathrm{z}$ calcd for $\mathrm{C}_{14} \mathrm{H}_{24} \mathrm{~S}_{2}\left[\mathrm{M}^{+}\right]$: 256.1319, found 256.1320 . 


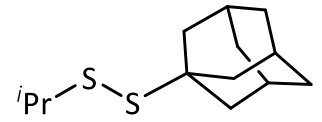

18

1-((1S,3R,5S)-adamantan-1-yl)-2-isopropyldisulfane (18): Following the synthetic procedure $\mathbf{D}, \mathbf{1 8}$ was obtained as a colorless oil (36.8 mg, 76\% yield). ${ }^{1} \mathrm{H}$ NMR (400 MHz, Chloroform-d) $\delta 2.90(\mathrm{p}, J=6.7 \mathrm{~Hz}, 1 \mathrm{H}$ ), $2.06(\mathrm{~s}, 3 \mathrm{H}), 1.85$ $(\mathrm{d}, J=3.0 \mathrm{~Hz}, 6 \mathrm{H}), 1.72-1.63(\mathrm{~m}, 6 \mathrm{H}), 1.28(\mathrm{~d}, J=6.7 \mathrm{~Hz}, 6 \mathrm{H}) .{ }^{13} \mathrm{C} \mathrm{NMR}(101 \mathrm{MHz}$, Chloroform-d) $\delta 49.1,42.8,42.4$, 36.3, 30.0, 22.7. HRMS (EI) $\mathrm{m} / \mathrm{z}$ calcd for $\mathrm{C}_{13} \mathrm{H}_{22} \mathrm{~S}_{2}\left[\mathrm{M}^{+}\right]$: 242.1163, found 242.1166.

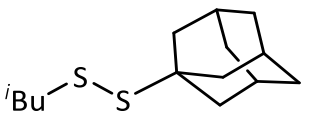

19

1-((1S,3R,5S)-adamantan-1-yl)-2-isobutyldisulfane (19): Following the synthetic procedure $\mathbf{C}$ (or D), 19 was obtained as a colorless oil (C: $38.5 \mathrm{mg}, 75 \%$ yield; D: $37.9 \mathrm{mg}, 74 \%$ yield). ${ }^{1} \mathrm{H}$ NMR (400 MHz, Chloroform-d) $\delta 2.59$ (d, $J=6.9$ $\mathrm{Hz}, 2 \mathrm{H}), 2.07(\mathrm{~s}, 3 \mathrm{H}), 1.92(\mathrm{dt}, J=13.4,6.7 \mathrm{~Hz}, 1 \mathrm{H}), 1.85(\mathrm{~d}, J=2.9 \mathrm{~Hz}, 6 \mathrm{H}), 1.72-1.64(\mathrm{~m}, 6 \mathrm{H}), 0.98(\mathrm{~d}, J=6.7 \mathrm{~Hz}, 6 \mathrm{H})$. ${ }^{13} \mathrm{C}$ NMR (101 MHz, Chloroform-d) $\delta 49.4,43.2,42.8,36.3,30.0,28.7,22.0$. HRMS (EI) m/z calcd for $\mathrm{C}_{14} \mathrm{H}_{24} \mathrm{~S}_{2}\left[\mathrm{M}^{+}\right]$: 256.1319, found 256.1317 .

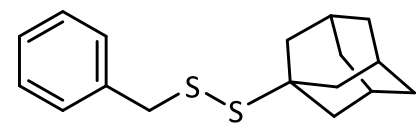

20

1-((1S,3R,5S)-adamantan-1-yl)-2-benzyldisulfane (20): Following the synthetic procedure $\mathbf{D}, \mathbf{2 0}$ was obtained as a colorless oil (35.4 mg, 61\% yield). ${ }^{1} \mathrm{H}$ NMR (300 MHz, Chloroform-d) $\delta 7.34-7.26(\mathrm{~m}, 5 \mathrm{H}), 3.91(\mathrm{~s}, 2 \mathrm{H}), 2.07(\mathrm{~s}, 3 \mathrm{H})$, $1.88(\mathrm{~d}, J=2.9 \mathrm{~Hz}, 6 \mathrm{H}), 1.75-1.65(\mathrm{~m}, 6 \mathrm{H}) .{ }^{13} \mathrm{C}$ NMR $(75 \mathrm{MHz}$, Chloroform-d) $\delta 137.6,129.4,128.6,127.5,49.8,46.4$, 42.8, 36.3, 30.0. HRMS (EI) $\mathrm{m} / \mathrm{z}$ calcd for $\mathrm{C}_{17} \mathrm{H}_{22} \mathrm{~S}_{2}\left[\mathrm{M}^{+}\right]$: 290.1163, found 290.1162 .

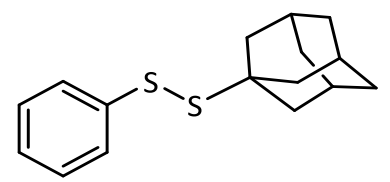

21

1-((1S,3R,5S)-adamantan-1-yl)-2-phenyldisulfane (21): Following the synthetic procedure $\mathbf{D}, \mathbf{2 1}$ was obtained as a light brown oil (37.0 mg, 67\% yield). ${ }^{1} \mathrm{H}$ NMR $(400 \mathrm{MHz}$, Chloroform-d) $\delta 7.55-7.53(\mathrm{~m}, 2 \mathrm{H}), 7.29-7.24(\mathrm{~m}, 2 \mathrm{H})$, $7.17-7.12(\mathrm{~m}, 1 \mathrm{H}), 2.01(\mathrm{~s}, 3 \mathrm{H}), 1.83(\mathrm{~d}, J=2.9 \mathrm{~Hz}, 6 \mathrm{H}), 1.68-1.58(\mathrm{~m}, 6 \mathrm{H}) .{ }^{13} \mathrm{C}$ NMR $(101 \mathrm{MHz}$, Chloroform-d) $\delta$ 139.4, 128.8, 126.6, 126.1, 50.9, 42.6, 36.2, 30.0. HRMS (EI) m/z calcd for $\mathrm{C}_{16} \mathrm{H}_{20} \mathrm{~S}_{2}\left[\mathrm{M}^{+}\right]: 276.1006$, found 276.1001.<smiles>CCCCCC(C)(C)SSC(C)(C)C</smiles>

22

1-trideuteriomethyl-2-(2-methylheptan-2-yl)disulfane (22): Following the synthetic procedure D, 22 was obtained as a colorless oil $\left(32.0 \mathrm{mg}, 82 \%\right.$ yield). ${ }^{1} \mathrm{H}$ NMR $(400 \mathrm{MHz}$, Chloroform- $d) \delta 1.58-1.53(\mathrm{~m}, 2 \mathrm{H}), 1.40-1.29(\mathrm{~m}, 4 \mathrm{H})$, 
$1.28(\mathrm{~s}, 6 \mathrm{H}), 1.27-1.22(\mathrm{~m}, 2 \mathrm{H}), 0.89(\mathrm{t}, J=7.1 \mathrm{~Hz}, 3 \mathrm{H}) .{ }^{13} \mathrm{C}$ NMR (101 MHz, Chloroform-d) $\delta$ 42.6, 41.7, 32.5, 27.8, 24.7, $24.54-24.47\left(m, C-D_{3}\right), 22.8,14.2$. HRMS (EI) m/z calcd for $\mathrm{C}_{9} \mathrm{H}_{17} \mathrm{D}_{3} \mathrm{~S}_{2}\left[\mathrm{M}^{+}\right]$: 195.1195, found 195.1198.

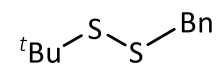

23

1-benzyl-2-(tert-butyl)disulfane (23): Following the synthetic procedure D, $\mathbf{2 3}$ was obtained as a colorless oil (27.6 $\mathrm{mg}, 65 \%$ yield). ${ }^{1} \mathrm{H}$ NMR $\left(400 \mathrm{MHz}\right.$, Chloroform-d) $\delta 7.34-7.18(\mathrm{~m}, 5 \mathrm{H}), 3.92(\mathrm{~s}, 2 \mathrm{H}), 1.33(\mathrm{~s}, 9 \mathrm{H}) .{ }^{13} \mathrm{C} \mathrm{NMR}(101 \mathrm{MHz}$, Chloroform-d) $\delta 137.5,129.4,128.7,127.5,48.2,45.9,30.2$. HRMS (EI) $\mathrm{m} / \mathrm{z}$ calcd for $\mathrm{C}_{11} \mathrm{H}_{16} \mathrm{~S}_{2}\left[\mathrm{M}^{+}\right]$: 212.0693, found 212.0695 .

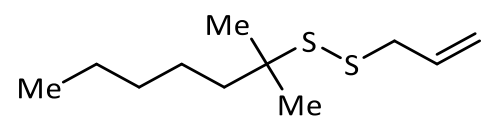

24

1-allyl-2-(2-methylheptan-2-yl)disulfane (24): Following the synthetic procedure $\mathbf{D}, \mathbf{2 4}$ was obtained as a colorless oil (31.4 mg, 72\% yield). ${ }^{1} \mathrm{H}$ NMR (400 MHz, Chloroform- $d$ ) $\delta 5.90-5.79(\mathrm{~m}, 1 \mathrm{H}), 5.19-5.11(\mathrm{~m}, 2 \mathrm{H}), 3.33(\mathrm{~d}, J=7.3$ $\mathrm{Hz}, 2 \mathrm{H}), 1.60-1.51(\mathrm{~m}, 2 \mathrm{H}), 1.38-1.24(\mathrm{~m}, 12 \mathrm{H}), 0.89(\mathrm{t}, J=7.0 \mathrm{~Hz}, 3 \mathrm{H}) .{ }^{13} \mathrm{C}$ NMR $(101 \mathrm{MHz}$, Chloroform-d) $\delta 133.6$, 118.4, 51.4, 44.0, 41.7, 32.5, 27.9, 24.6, 22.8, 14.2. HRMS (EI) $\mathrm{m} / \mathrm{z}$ calcd for $\mathrm{C}_{11} \mathrm{H}_{22} \mathrm{~S}_{2}\left[\mathrm{M}^{+}\right]$: 218.1163, found 218.1166 .

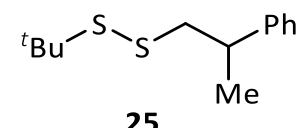

1-(tert-butyl)-2-(2-phenylpropyl)disulfane (25): Following the synthetic procedure $\mathbf{D}, \mathbf{2 5}$ was obtained as a colorless oil (40.9 mg, 85\% yield). ${ }^{1} \mathrm{H}$ NMR (400 MHz, Chloroform- $d$ ) $\delta 7.35-7.30(\mathrm{~m}, 2 \mathrm{H}), 7.24-7.18(\mathrm{~m}, 3 \mathrm{H}), 3.13$ $-3.01(\mathrm{~m}, 2 \mathrm{H}), 2.86(\mathrm{dd}, J=12.4,8.3 \mathrm{~Hz}, 1 \mathrm{H}), 1.37(\mathrm{~d}, J=6.9 \mathrm{~Hz}, 3 \mathrm{H}), 1.33(\mathrm{~s}, 9 \mathrm{H}) .{ }^{13} \mathrm{C}$ NMR $(101 \mathrm{MHz}$, Chloroform-d) $\delta 145.6,128.6,127.2,126.6,49.7,48.0,30.1,20.9,20.7$. HRMS (EI) $\mathrm{m} / \mathrm{z}$ calcd for $\mathrm{C}_{13} \mathrm{H}_{20} \mathrm{~S}_{2}\left[\mathrm{M}^{+}\right]: 240.1006$, found 240.1003.<smiles>CC(C)(C)SSCCCCBr</smiles>

26

1-(4-bromobutyl)-2-(tert-butyl)disulfane (26): Following the synthetic procedure D, 26 was obtained as a colorless oil (44.8 mg, 87\% yield). ${ }^{1} \mathrm{H}$ NMR (400 MHz, Chloroform- $d$ ) $\delta 3.42(\mathrm{t}, J=6.6 \mathrm{~Hz}, 2 \mathrm{H}$ ), $2.72(\mathrm{t}, J=7.1 \mathrm{~Hz}, 2 \mathrm{H}$ ), $1.99-$ $1.93(\mathrm{~m}, 2 \mathrm{H}), 1.86-1.81(\mathrm{~m}, 2 \mathrm{H}), 1.33(\mathrm{~s}, 9 \mathrm{H}) .{ }^{13} \mathrm{C}$ NMR (101 MHz, Chloroform-d) $\delta$ 48.0, 39.8, 33.3, 31.5, 30.1, 27.8. HRMS (EI) $\mathrm{m} / \mathrm{z}$ calcd for $\mathrm{C}_{8} \mathrm{H}_{17} \mathrm{BrS}_{2}\left[\mathrm{M}^{+}\right]: 255.9955,257.9935$, found 255.9955, 257.9933.<smiles>CC(C)(C)CSSCCC(=O)c1ccccc1</smiles>

27

3-(tert-butyldisulfanyl)-1-phenylpropan-1-one (27): Following the synthetic procedure $\mathbf{D}, \mathbf{2 7}$ was obtained as a colorless oil (40.7 mg, 80\% yield). ${ }^{1} \mathrm{H}$ NMR (300 MHz, Chloroform- $d$ ) $\delta 8.00-7.97(\mathrm{~m}, 2 \mathrm{H}), 7.61-7.55(\mathrm{~m}, 1 \mathrm{H}), 7.50$ 
- $7.45(\mathrm{~m}, 2 \mathrm{H}), 3.40(\mathrm{t}, J=7.3 \mathrm{~Hz}, 2 \mathrm{H}), 3.07(\mathrm{t}, J=7.4 \mathrm{~Hz}, 2 \mathrm{H}), 1.35(\mathrm{~s}, 9 \mathrm{H}) .{ }^{13} \mathrm{C}$ NMR $(75 \mathrm{MHz}$, Chloroform-d) $\delta$ 198.4, 136.7, 133.5, 128.8, 128.2, 48.2, 38.8, 34.5, 30.1. HRMS (EI) $\mathrm{m} / \mathrm{z}$ calcd for $\mathrm{C}_{13} \mathrm{H}_{18} \mathrm{OS}_{2}\left[\mathrm{M}^{+}\right]$: 254.0799, found 254.0798.

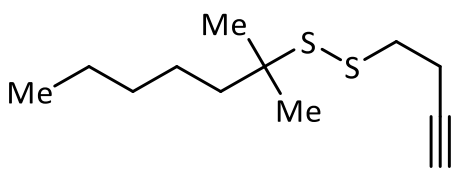

28

1-(but-3-yn-1-yl)-2-(2-methylheptan-2-yl)disulfane (28): Following the synthetic procedure D, 28 was obtained as a colorless oil ( $36.4 \mathrm{mg}, 79 \%$ yield). ${ }^{1} \mathrm{H}$ NMR ( $400 \mathrm{MHz}$, Chloroform- $d$ ) $\delta 2.80(\mathrm{t}, J=7.6 \mathrm{~Hz}, 2 \mathrm{H}$ ), 2.58 (ddd, $J=7.9$, 6.9, $2.7 \mathrm{~Hz}, 2 \mathrm{H}), 2.02(\mathrm{t}, J=2.6 \mathrm{~Hz}, 1 \mathrm{H}), 1.57-1.55(\mathrm{~m}, 2 \mathrm{H}), 1.35-1.29(\mathrm{~m}, 4 \mathrm{H}), 1.28(\mathrm{~s}, 6 \mathrm{H}), 1.27-1.25(\mathrm{~m}, 2 \mathrm{H}), 0.89$ $(\mathrm{t}, J=7.1 \mathrm{~Hz}, 3 \mathrm{H}) .{ }^{13} \mathrm{C}$ NMR $(101 \mathrm{MHz}$, Chloroform-d) $\delta 82.5,69.5,51.5,41.7,38.5,32.4,27.8,24.6,22.8,19.3,14.2$. HRMS (EI) $\mathrm{m} / \mathrm{z}$ calcd for $\mathrm{C}_{12} \mathrm{H}_{22} \mathrm{~S}_{2}\left[\mathrm{M}^{+}\right]: 230.1163$, found 230.1153 .

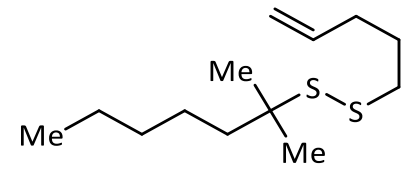

29

1-(2-methylheptan-2-yl)-2-(pent-4-en-1-yl)disulfane (29): Following the synthetic procedure D, 29 was obtained as a colorless oil (41.4 mg, 84\% yield). ${ }^{1} \mathrm{H}$ NMR (400 MHz, Chloroform-d) $\delta 5.78$ (ddt, $J=16.9,10.2,6.7 \mathrm{~Hz}, 1 \mathrm{H}$ ), $5.10-4.92(\mathrm{~m}, 2 \mathrm{H}), 2.67(\mathrm{t}, J=7.4 \mathrm{~Hz}, 2 \mathrm{H}), 2.17-2.10(\mathrm{~m}, 2 \mathrm{H}), 1.76(\mathrm{p}, J=7.3 \mathrm{~Hz}, 2 \mathrm{H}), 1.55-1.52(\mathrm{~m}, 2 \mathrm{H}), 1.39-$ $1.28(\mathrm{~m}, 5 \mathrm{H}), 1.27(\mathrm{~s}, 6 \mathrm{H}), 1.25-1.23(\mathrm{~m}, 1 \mathrm{H}), 0.89(\mathrm{t}, J=7.0 \mathrm{~Hz}, 3 \mathrm{H}) .{ }^{13} \mathrm{C}$ NMR (101 MHz, Chloroform-d) $\delta 137.8$, 115.4, 51.2, 41.6, 39.9, 32.6, 32.5, 28.6, 27.9, 24.6, 22.8, 14.2. HRMS (EI) m/z calcd for $\mathrm{C}_{13} \mathrm{H}_{26} \mathrm{~S}_{2}\left[\mathrm{M}^{+}\right]: 246.1476$, found 246.1477 .

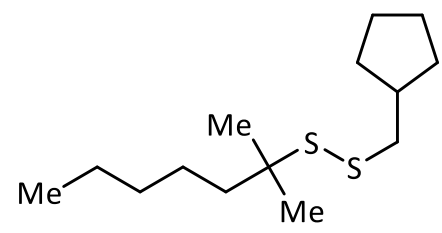

30

1-(cyclopentylmethyl)-2-(2-methylheptan-2-yl)disulfane (30): Following the synthetic procedure D, 30 was obtained as a colorless oil ( $34.9 \mathrm{mg}, 67 \%$ yield). ${ }^{1} \mathrm{H}$ NMR $(400 \mathrm{MHz}$, Chloroform- $d$ ) $\delta 2.71$ (dd, $J=7.3,3.1 \mathrm{~Hz}, 2 \mathrm{H})$, $2.23-2.09(\mathrm{~m}, 1 \mathrm{H}), 1.85-1.80(\mathrm{~m}, 2 \mathrm{H}), 1.64-1.52(\mathrm{~m}, 6 \mathrm{H}), 1.37-1.28(\mathrm{~m}, 6 \mathrm{H}), 1.27(\mathrm{~s}, 6 \mathrm{H}), 1.26-1.21(\mathrm{~m}, 2 \mathrm{H})$, $0.89(\mathrm{t}, J=7.0 \mathrm{~Hz}, 3 \mathrm{H}) .{ }^{13} \mathrm{C}$ NMR $(101 \mathrm{MHz}$, Chloroform-d) $\delta 51.2,47.6,45.8,41.6,39.9,32.5,32.3,32.3,27.9,25.4$, 25.3, 24.6, 22.8, 14.2. HRMS (EI) $\mathrm{m} / \mathrm{z}$ calcd for $\mathrm{C}_{14} \mathrm{H}_{28} \mathrm{~S}_{2}\left[\mathrm{M}^{+}\right]: 260.1632$, found 260.1630 .

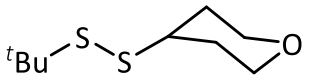

31

4-(tert-butyldisulfanyl)tetrahydro-2H-pyran (31): Following the synthetic procedure $\mathbf{D}, \mathbf{3 1}$ was obtained as a colorless oil (34.2 mg, 83\% yield). ${ }^{1} \mathrm{H}$ NMR (300 MHz, Chloroform- $d$ ) $\delta 3.99$ (dt, $J=11.8,3.8 \mathrm{~Hz}, 2 \mathrm{H}$ ), 3.40 (ddd, $J=$ 
11.4, 10.7, $2.4 \mathrm{~Hz}, 2 \mathrm{H}), 2.89(\mathrm{tt}, J=10.8,4.1 \mathrm{~Hz}, 1 \mathrm{H}), 2.05-1.98(\mathrm{~m}, 2 \mathrm{H}), 1.67-1.54(\mathrm{~m}, 2 \mathrm{H}), 1.33(\mathrm{~s}, 9 \mathrm{H}) .{ }^{13} \mathrm{C} \mathrm{NMR}$ (75 MHz, Chloroform-d) $\delta 67.5,47.6,46.9,32.9,30.2$. HRMS (EI) m/z calcd for $\mathrm{C}_{9} \mathrm{H}_{18} \mathrm{OS}_{2}\left[\mathrm{M}^{+}\right]$: 206.0799, found 206.0792.

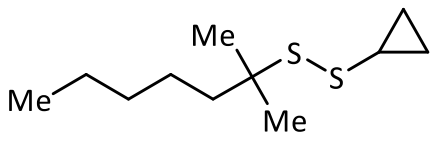

32

1-cyclopropyl-2-(2-methylheptan-2-yl)disulfane (32): Following the synthetic procedure $\mathbf{D}, \mathbf{3 2}$ was obtained as a colorless oil (32.8 mg, 75\% yield). ${ }^{1} \mathrm{H}$ NMR (400 MHz, Chloroform-d) $\delta 2.19-2.13(\mathrm{~m}, 1 \mathrm{H}), 1.60-1.54(\mathrm{~m}, 3 \mathrm{H}), 1.42$ - $1.32(\mathrm{~m}, 3 \mathrm{H}), 1.31(\mathrm{~s}, 6 \mathrm{H}), 1.29-1.23(\mathrm{~m}, 2 \mathrm{H}), 0.93-0.87(\mathrm{~m}, 5 \mathrm{H}), 0.75-0.69(\mathrm{~m}, 2 \mathrm{H}) .{ }^{13} \mathrm{C}$ NMR $(101 \mathrm{MHz}$, Chloroform-d) $\delta 50.8,42.0,32.5,31.1,28.1,24.6,22.8,20.3,14.2,10.3$. HRMS (EI) $\mathrm{m} / \mathrm{z}$ calcd for $\mathrm{C}_{11} \mathrm{H}_{22} \mathrm{~S}_{2}\left[\mathrm{M}^{+}\right]$: 218.1163, found 218.1165 .

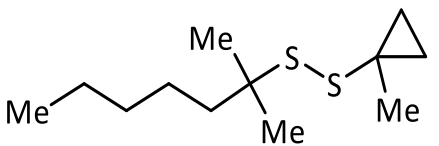

33

1-(1-methylcyclopropyl)-2-(2-methylheptan-2-yl)disulfane (33): Following the synthetic procedure D, 33 was obtained as a colorless oil (35.8 mg, 77\% yield). ${ }^{1} \mathrm{H}$ NMR (400 MHz, Chloroform-d) $\delta 1.56-1.53(\mathrm{~m}, 2 \mathrm{H}), 1.45(\mathrm{~s}$, $3 \mathrm{H}), 1.41-1.30(\mathrm{~m}, 4 \mathrm{H}), 1.28(\mathrm{~s}, 6 \mathrm{H}), 1.26-1.23(\mathrm{~m}, 1 \mathrm{H}), 0.96-0.91(\mathrm{~m}, 1 \mathrm{H}), 0.91-0.87(\mathrm{~m}, 4 \mathrm{H}), 0.75-0.70(\mathrm{~m}$, 3H). ${ }^{13} \mathrm{C}$ NMR (101 MHz, Chloroform-d) $\delta 49.9,42.4,32.4,28.4,25.2,25.1,24.6,22.8,17.7,17.6,14.2$. HRMS (EI) $\mathrm{m} / \mathrm{z}$ calcd for $\mathrm{C}_{12} \mathrm{H}_{24} \mathrm{~S}_{2}\left[\mathrm{M}^{+}\right]$: 232.1319, found 232.1317.

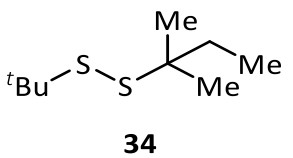

1-(tert-butyl)-2-(tert-pentyl)disulfane (34): Following the synthetic procedure $\mathbf{D}, \mathbf{3 4}$ was obtained as a colorless oil (28.5 mg, 74\% yield). ${ }^{1} \mathrm{H}$ NMR (400 MHz, Chloroform-d) $\delta 1.57$ (q, $J=7.4 \mathrm{~Hz}, 2 \mathrm{H}$ ), $1.30(\mathrm{~s}, 9 \mathrm{H}), 1.24(\mathrm{~s}, 6 \mathrm{H}$ ), 0.93 (t, $J=7.4 \mathrm{~Hz}, 3 \mathrm{H}) .{ }^{13} \mathrm{C}$ NMR $(101 \mathrm{MHz}$, Chloroform-d) $\delta 50.1,46.3,34.9,30.7,27.8,9.4$. HRMS (EI) $\mathrm{m} / \mathrm{z}$ calcd for $\mathrm{C}_{9} \mathrm{H}_{20} \mathrm{~S}_{2}\left[\mathrm{M}^{+}\right]:$192.1006, found 192.1017 .

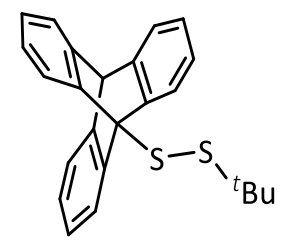

35

1-(tert-butyl)-2-(9,10-dihydro-9,10-[1,2]benzenoanthracen-9-yl)disulfane (35): Following the synthetic procedure D, 35 was obtained as a white solid (19.5 mg, 26\% yield). ${ }^{1} \mathrm{H}$ NMR (400 MHz, Chloroform-d) $\delta 7.83-7.80(\mathrm{~m}, 3 \mathrm{H})$, $7.38-7.26(\mathrm{~m}, 3 \mathrm{H}), 7.07-7.00(\mathrm{~m}, 6 \mathrm{H}), 5.34(\mathrm{~s}, 1 \mathrm{H}), 1.69(\mathrm{~s}, 9 \mathrm{H}) .{ }^{13} \mathrm{C}$ NMR $(101 \mathrm{MHz}$, Chloroform-d) $\delta$ 145.6, 144.4, 125.7, 124.9, 123.5, 123.4, 64.4, 54.1, 49.0, 31.2. HRMS (EI) m/z calcd for $\mathrm{C}_{24} \mathrm{H}_{22} \mathrm{~S}_{2}\left[\mathrm{M}^{+}\right]: 374.1163$, found 374.1168. 
<smiles>CC(C)(C)SSc1ccc(Br)cc1</smiles>

36

1-(4-bromophenyl)-2-(tert-butyl)disulfane (36): Following the synthetic procedure D (0.1 mmol bis(4fluorophenyl)methanone 0-(4-bromobenzoyl) oxime and $0.5 \mathrm{mmol}$ 1,4-di-tert-butyltetrasulfane were used), 36 was obtained as a colorless oil $\left(4.7 \mathrm{mg}, 17 \%\right.$ yield). ${ }^{1} \mathrm{H}$ NMR (400 MHz, Chloroform-d) $\delta 7.44-7.26(\mathrm{~m}, 5 \mathrm{H})$, 1.30 (s, 9H). ${ }^{13} \mathrm{C}$ NMR (101 MHz, Chloroform-d) $\delta$ 138.3, 131.9, 128.4, 120.06, 49.6, 30.0. HRMS (EI) m/z calcd for $\mathrm{C}_{10} \mathrm{H}_{13} \mathrm{BrS}_{2}\left[\mathrm{M}^{+}\right]: 275.9642$, found 275.9543.

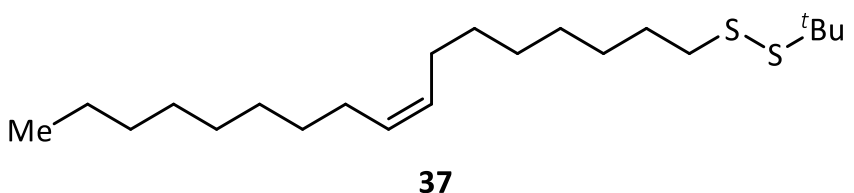

(Z)-1-(tert-butyl)-2-(heptadec-8-en-1-yl)disulfane (37): Following the synthetic procedure D, 37 was obtained as a colorless oil (56.7 mg, 79\% yield). ${ }^{1} \mathrm{H}$ NMR (400 MHz, Chloroform- $d$ ) $\delta 5.41-5.36(\mathrm{~m}, 1 \mathrm{H}), 5.36-5.29(\mathrm{~m}, 1 \mathrm{H}), 2.70$ $(\mathrm{t}, J=7.6 \mathrm{~Hz}, 2 \mathrm{H}), 2.03-1.94(\mathrm{~m}, 4 \mathrm{H}), 1.68-1.94(\mathrm{~m}, 2 \mathrm{H}), 1.39-1.26(\mathrm{~m}, 31 \mathrm{H}), 0.89-0.86(\mathrm{~m}, 3 \mathrm{H}) .{ }^{13} \mathrm{C} \mathrm{NMR}(101$ MHz, Chloroform-d) $\delta$ 130.4, 130.2, 47.8, 41.1, 32.8, 32.1, 30.1, 29.9, 29.8, 29.7, 29.7, 29.5, 29.3, 29.3, 29.2, 29.1, 28.7, 27.3, 22.8, 14.3. HRMS (EI) m/z calcd for $\mathrm{C}_{21} \mathrm{H}_{42} \mathrm{~S}_{2}\left[\mathrm{M}^{+}\right]$: 358.2728, found 358.2731.<smiles>CC(=O)[C@H]1C[C@H](CSSCC(C)C)C1(C)C</smiles>

38

1-((1R,3S)-3-((tert-butyldisulfanyl)methyl)-2,2-dimethylcyclobutyl)ethanone (38): Following the synthetic procedure D, 38 was obtained as a colorless oil (13.0 mg, 25\% yield). ${ }^{1} \mathrm{H}$ NMR (400 MHz, Chloroform-d) $\delta 2.83$ $(\mathrm{dd}, J=10.1,7.6 \mathrm{~Hz}, 1 \mathrm{H}), 2.75-2.61(\mathrm{~m}, 2 \mathrm{H}), 2.29(\mathrm{dtd}, J=10.4,8.3,7.2 \mathrm{~Hz}, 1 \mathrm{H}), 2.04(\mathrm{~s}, 3 \mathrm{H}), 1.99-1.90(\mathrm{~m}, 2 \mathrm{H})$, 1.36 (s, 3H), 1.32 (s, 9H), 0.91 (s, 3H). ${ }^{13} \mathrm{C}$ NMR (151 MHz, Chloroform-d) $\delta$ 207.8, 53.8, 48.0, 43.8, 41.8, 41.0, 30.8, 30.4, 30.1, 23.3, 17.2. HRMS (EI) m/z calcd for $\mathrm{C}_{13} \mathrm{H}_{24} \mathrm{OS}_{2}\left[\mathrm{M}^{+}\right]: 260.1269$, found 260.1266.

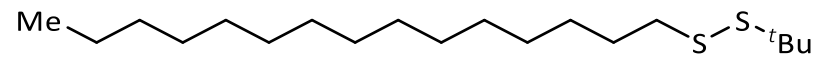

39

1-(tert-butyl)-2-pentadecyldisulfane (39): Following the synthetic procedure D, 39 was obtained as a colorless oil (58.5 mg, 88\% yield). ${ }^{1} \mathrm{H}$ NMR (400 MHz, Chloroform-d) $\delta 2.70$ (t, J=7.4 Hz, 2H), 1.64 (p, J= 7.4 Hz, $2 \mathrm{H}$ ), $1.40-1.35$ $(\mathrm{m}, 2 \mathrm{H}), 1.34-1.31(\mathrm{~m}, 9 \mathrm{H}), 1.31-1.22(\mathrm{~m}, 23 \mathrm{H}), 0.88(\mathrm{t}, J=6.7 \mathrm{~Hz}, 3 \mathrm{H}) .{ }^{13} \mathrm{C}$ NMR $(101 \mathrm{MHz}, \mathrm{Chloroform}-d) \delta 47.8$, 41.2, 32.1, 30.7, 30.1, 29.9, 29.8, 29.8, 29.7, 29.5, 29.5, 29.4, 28.8, 22.9, 14.3. HRMS (EI) m/z calcd for $\mathrm{C}_{19} \mathrm{H}_{40} \mathrm{~S}_{2}\left[\mathrm{M}^{+}\right]$: 332.2571, found 332.2570. 


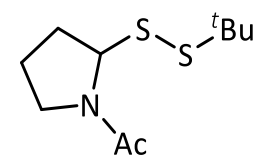

40

1-(2-(tert-butyldisulfanyl)pyrrolidin-1-yl)ethanone (40): Following the synthetic procedure $\mathbf{D}, \mathbf{4 0}$ was obtained as a white solid (26.1 mg, 56\% yield). mixture of ratomers (1:0.8), ${ }^{1} \mathrm{H} \mathrm{NMR} \mathrm{(400} \mathrm{MHz,} \mathrm{Chloroform-d)} \delta$ major: 4.92 (d, $J=5.6 \mathrm{~Hz}, 1 \mathrm{H}), 3.45-3.34(\mathrm{~m}, 2 \mathrm{H}), 2.48-2.42(\mathrm{~m}, 1 \mathrm{H}), 2.31(\mathrm{~s}, 3 \mathrm{H}), 2.28-2.25(\mathrm{~m}, 1 \mathrm{H}), 2.20-2.15(\mathrm{~m}, 2 \mathrm{H}), 1.35(\mathrm{~s}$, $9 \mathrm{H})$; minor: $5.26(\mathrm{~d}, J=6.0 \mathrm{~Hz}, 1 \mathrm{H}), 3.60-3.55(\mathrm{~m}, 2 \mathrm{H}), 2.36-2.34(\mathrm{~m}, 1 \mathrm{H}), 2.12-2.07(\mathrm{~m}, 1 \mathrm{H}), 2.06(\mathrm{~s}, 3 \mathrm{H}), 2.02-$ 1.90 (m, 2H), 1.38 (s, 9H). ${ }^{13} \mathrm{C}$ NMR (101 MHz, Chloroform-d) $\delta$ 169.9, 169.5, 69.5, 67.1, 48.4, 48.2, 47.9, 46.5, 33.1, 31.7, 30.4, 30.2, 23.1, 23.0, 22.8, 21.2. HRMS (EI) m/z calcd for $\mathrm{C}_{10} \mathrm{H}_{19} \mathrm{NOS}_{2}\left[\mathrm{M}^{+}\right]: 233.0908$, found 233.0911 .<smiles>CC(=O)OC[C@@H](CSSCC(C)C)C(C)=O</smiles>

41

(R)-methyl 2-(N,N-di-(tert-butoxycarbonyl)amino)-3-(tert-butyldisulfanyl)propanoate (41): Following the synthetic procedure D, 41 was obtained as a colorless oil (60.1 mg, 71\% yield). ${ }^{1} \mathrm{H}$ NMR (300 MHz, Chloroform-d) $\delta 5.23$ (dd, $J=9.6,4.6 \mathrm{~Hz}, 1 \mathrm{H}$ ), $3.72(\mathrm{~s}, 3 \mathrm{H}), 3.45$ (dd, $J=14.1,4.6 \mathrm{~Hz}, 1 \mathrm{H}), 3.18(\mathrm{dd}, J=14.1,9.7 \mathrm{~Hz}, 1 \mathrm{H}), 1.54-1.46(\mathrm{~m}$, 18H), 1.33 (s, 9H). ${ }^{13} \mathrm{C} \mathrm{NMR}(75 \mathrm{MHz}$, Chloroform-d) $\delta$ 170.5, 151.9, 83.5, 58.0, 52.5, 48.1, 41.2, 30.0, 28.1. HRMS (EI) $\mathrm{m} / \mathrm{z}$ calcd for $\mathrm{C}_{18} \mathrm{H}_{33} \mathrm{NO}_{6} \mathrm{~S}_{2}\left[\mathrm{M}^{+}\right]: 423.1749$, found 423.1755 .<smiles>CCCCN[C@@H](CCSSC(C)C)C(C)=O</smiles>

(S)-methyl 2-(N,N-di-(tert-butoxycarbonyl)amino)-4-(tert-butyldisulfanyl)butanoate (42): Following the synthetic procedure D, 42 was obtained as a colorless oil (63.9 mg, 73\% yield). ${ }^{1} \mathrm{H}$ NMR (300 MHz, Chloroform- $d$ ) $\delta 4.98-$ $4.94(\mathrm{~m}, 1 \mathrm{H}), 3.68(\mathrm{~s}, 3 \mathrm{H}), 2.72-2.67(\mathrm{~m}, 2 \mathrm{H}), 2.55-2.50(\mathrm{~m}, 1 \mathrm{H}), 2.49-2.46(\mathrm{~m}, 1 \mathrm{H}), 2.24-1.46(\mathrm{~m}, 18 \mathrm{H}), 1.28(\mathrm{~s}$, 9H). ${ }^{13} \mathrm{C}$ NMR (75 MHz, Chloroform-d) $\delta$ 171.0, 151.9, 83.4, 57.0, 52.4, 48.0, 37.1, 30.1, 30.0, 28.1. HRMS (EI) m/z calcd for $\mathrm{C}_{19} \mathrm{H}_{35} \mathrm{NO}_{6} \mathrm{~S}_{2}\left[\mathrm{M}^{+}\right]$: 437.1906, found 437.1913 .

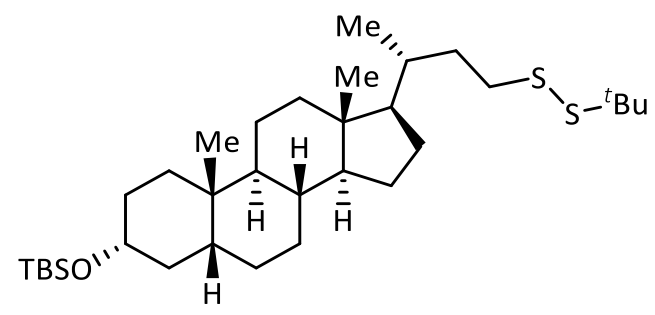

43

tert-butyl(((3R,5R,8R,9S,10S,13R,14S,17R)-17-((R)-4-(tert-butyldisulfanyl)butan-2-yl)-10,13-

dimethylhexadecahydro- $1 \mathrm{H}$-cyclopenta[a]phenanthren-3-yl)oxy)dimethylsilane (43): Following the synthetic procedure D, 43 was obtained as a white solid $\left(69.2 \mathrm{mg}, 61 \%\right.$ yield). ${ }^{1} \mathrm{H}$ NMR (400 MHz, Chloroform- $d$ ) $\delta 3.56$ (tt, $J=10.5,4.7 \mathrm{~Hz}, 1 \mathrm{H}), 1.97-1.88(\mathrm{~m}, 1 \mathrm{H}), 1.86-1.65(\mathrm{~m}, 4 \mathrm{H}), 1.54(\mathrm{~s}, 7 \mathrm{H}), 1.49-0.91(\mathrm{~m}, 20 \mathrm{H}), 0.87(\mathrm{~d}, J=2.3 \mathrm{~Hz}$, 
$16 \mathrm{H}), 0.80(\mathrm{t}, J=7.4 \mathrm{~Hz}, 3 \mathrm{H}), 0.61(\mathrm{~s}, 3 \mathrm{H}), 0.04(\mathrm{~s}, 6 \mathrm{H}) .{ }^{13} \mathrm{C}$ NMR (101 MHz, Chloroform-d) $\delta$ 73.0, 56.6, 56.0, 42.8, $42.5,40.4,40.3,37.2$, 37.1, 36.0, 35.7, 34.7, 31.2, 28.4, 28.4, 27.5, 26.6, 26.1, 24.4, 23.6, 21.0, 18.5, 18.2, 12.2, 10.5, -4.4. HRMS (EI) $\mathrm{m} / \mathrm{z}$ calcd for $\mathrm{C}_{33} \mathrm{H}_{62} \mathrm{OS}_{2} \mathrm{Si}\left[\mathrm{M}^{+}\right]: 566.4011$, found 566.4009 .

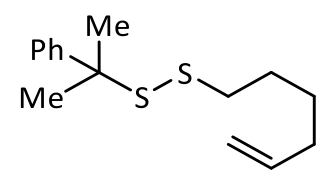

47

1-(hex-5-en-1-yl)-2-(2-phenylpropan-2-yl)disulfane (47): Following the synthetic procedure $\mathbf{D}, \mathbf{4 7}$ was obtained as a colorless oil ( $8.0 \mathrm{mg}, 15 \%$ isolated yield). ${ }^{1} \mathrm{H}$ NMR (400 MHz, Chloroform- $d$ ) $\delta 7.51-7.49(\mathrm{~m}, 2 \mathrm{H}), 7.32-7.27(\mathrm{~m}$, $2 \mathrm{H}), 7.24-7.20(\mathrm{~m}, 1 \mathrm{H}), 5.73(\mathrm{ddt}, J=16.9,10.2,6.6 \mathrm{~Hz}, 1 \mathrm{H}), 4.98-4.90(\mathrm{~m}, 2 \mathrm{H}), 2.10(\mathrm{t}, J=7.4 \mathrm{~Hz}, 2 \mathrm{H}), 1.98-1.91$ $(\mathrm{m}, 2 \mathrm{H}), 1.73(\mathrm{~s}, 6 \mathrm{H}), 1.47-1.40(\mathrm{~m}, 2 \mathrm{H}), 1.32-1.23(\mathrm{~m}, 2 \mathrm{H}) .{ }^{13} \mathrm{C}$ NMR (101 MHz, Chloroform-d) $\delta$ 145.4, 138.5, 128.1, 126.9, 126.6, 114.6, 52.1, 38.7, 33.2, 28.5, 27.7. HRMS (EI) m/z calcd for $\mathrm{C}_{15} \mathrm{H}_{22} \mathrm{~S}_{2}\left[\mathrm{M}^{+}\right]$: 266.1163, found 266.1160 .

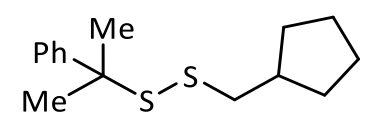

48

1-(cyclopentylmethyl)-2-(2-phenylpropan-2-yl)disulfane (48): Following the synthetic procedure D, 48 was obtained as a colorless oil ( $36.8 \mathrm{mg}, 69 \%$ isolated yield). ${ }^{1} \mathrm{H}$ NMR (400 MHz, Chloroform-d) $\delta 7.52-7.49(\mathrm{~m}, 2 \mathrm{H})$, $7.32-7.28(\mathrm{~m}, 2 \mathrm{H}), 7.24-7.20(\mathrm{~m}, 1 \mathrm{H}), 2.15(\mathrm{~d}, J=7.3 \mathrm{~Hz}, 2 \mathrm{H}), 1.92(\mathrm{dt}, J=15.2,7.6 \mathrm{~Hz}, 1 \mathrm{H}), 1.74(\mathrm{~s}, 6 \mathrm{H}), 1.66(\mathrm{tdd}$, $J=10.1,5.1,3.6 \mathrm{~Hz}, 2 \mathrm{H}), 1.56-1.40(\mathrm{~m}, 4 \mathrm{H}), 1.10-0.98(\mathrm{~m}, 2 \mathrm{H}) .{ }^{13} \mathrm{C}$ NMR $(101 \mathrm{MHz}$, Chloroform-d) $\delta 145.5,128.2$, 127.0, 126.8, 52.2, 45.7, 39.5, 32.2, 28.6, 25.3. HRMS (EI) m/z calcd for $\mathrm{C}_{15} \mathrm{H}_{22} \mathrm{~S}_{2}\left[\mathrm{M}^{+}\right]$: 266.1163, found 266.1160 .<smiles>CC(=O)SSSSC(C)=O</smiles>

1,1'-tetrasulfanediyldiethanone (51): Following the literature procedure ${ }^{4 \mathrm{~d}}$, A solution of triethylamine (1.62 ml, $12 \mathrm{mmol})$ in dry tetrahydrofuran $(10 \mathrm{ml})$ was added dropwise over a period of $20 \mathrm{~min}$ to a stirred solution of ethanethioic S-acid (913 $\mathrm{mg}, 12 \mathrm{mmol}$ ) and sulphur monochloride $(0.6 \mathrm{ml}, 7.5 \mathrm{mmol})$ in dry tetrahydrofuran (15 $\mathrm{ml}$ ) under ice-water bath. The reaction was stirred for a further period of $1 \mathrm{hr}$ at room temperature, and were then filtered. The filtrate was concentrated under reduced pressure, and the residue was extracted with warm ethyl acetate $(20 \mathrm{ml})$. After filtration, the extract was cooled and concentrated under reduced pressure. 51 was obtained as light-yellow oil (1.09 g, 85\% yield). ${ }^{1} \mathrm{H}$ NMR (300 MHz, Chloroform-d) $\delta 2.49(\mathrm{~s}, 3 \mathrm{H}) .{ }^{13} \mathrm{C} \mathrm{NMR}$ (75 MHz, Chloroform-d) $\delta$ 191.5, 29.4. HRMS (EI) m/z calcd for $\mathrm{C}_{4} \mathrm{H}_{6} \mathrm{O}_{2} \mathrm{~S}_{4}\left[\mathrm{M}^{+}\right]: 213.9251$, found 213.9253.

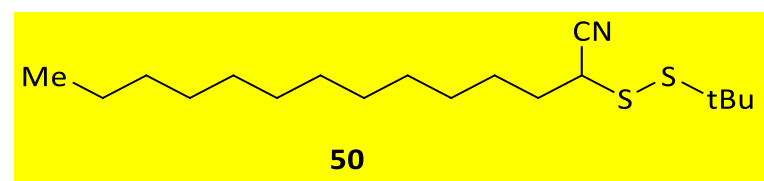

2-(tert-butyldisulfanyl)tetradecanenitrile (50): colorless oil $\left(16.8 \mathrm{mg}, 51 \%\right.$ yield). ${ }^{1} \mathrm{H}$ NMR (400 MHz, Chloroform-d) $\delta 3.52(\mathrm{dd}, J=7.7,7.0 \mathrm{~Hz}, 1 \mathrm{H}), 1.90-1.84(\mathrm{~m}, 2 \mathrm{H}), 1.63-1.56(\mathrm{~m}, 1 \mathrm{H}), 1.54-1.48(\mathrm{~m}, 1 \mathrm{H}), 1.38$ 
(s, 9H), $1.32-1.26(\mathrm{~m}, 18 \mathrm{H}), 0.88(\mathrm{t}, J=6.0 \mathrm{~Hz}, 3 \mathrm{H}) .{ }^{13} \mathrm{C}$ NMR (101 MHz, Chloroform-d) $\delta$ 119.4, 49.0, 41.4, 32.7, $32.1,30.3,29.8,29.7,29.6,29.5,29.4,29.0,27.1,22.8,14.3$. HRMS (EI) $\mathrm{m} / \mathrm{z}$ calcd for $\mathrm{C}_{18} \mathrm{H}_{35} \mathrm{NS}_{2}\left[\mathrm{M}^{+}\right]: 329.2211$, found 329.2211 .

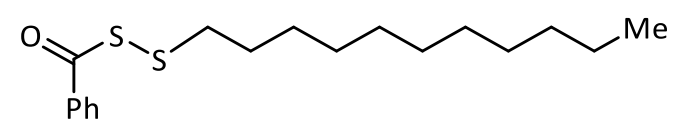

53

SS-undecyl benzo(dithioperoxoate) (53): white solid (40.9 mg, 63\% yield). ${ }^{1} \mathrm{H}$ NMR (300 MHz, Chloroform- $d$ ) $\delta 8.01$ - $7.98(\mathrm{~m}, 2 \mathrm{H}), 7.65-7.59(\mathrm{~m}, 1 \mathrm{H}), 7.51-7.45(\mathrm{~m}, 2 \mathrm{H}), 2.78(\mathrm{t}, J=6.7 \mathrm{~Hz}, 2 \mathrm{H}), 1.72-1.62(\mathrm{~m}, 2 \mathrm{H}), 1.43-1.36(\mathrm{~m}$, 2H), $1.29-1.23(\mathrm{~m}, 14 \mathrm{H}), 0.88(\mathrm{t}, J=6.7 \mathrm{~Hz}, 3 \mathrm{H}) .{ }^{13} \mathrm{C} \mathrm{NMR}(75 \mathrm{MHz}$, Chloroform-d) $\delta 190.6,136.0,134.1,129.0,127.8$, $38.9,32.1,29.7,29.7,29.6,29.5,29.3,29.2,28.6,22.8,14.3$. HRMS (EI) $\mathrm{m} / \mathrm{z}$ calcd for $\mathrm{C}_{18} \mathrm{H}_{28} \mathrm{OS}_{2}\left[\mathrm{M}^{+}\right]: 324.1582$, found 324.1584 .

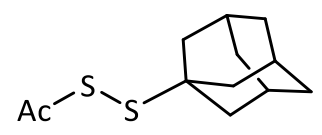

55

SS-(1S,3R,5S)-adamantan-1-yl ethane(dithioperoxoate) (55): colorless oil ( $23.3 \mathrm{mg}, 48 \%$ yield). ${ }^{1} \mathrm{H}$ NMR (300 MHz, Chloroform-d) $\delta 2.45(\mathrm{~s}, 3 \mathrm{H}), 2.11-2.03(\mathrm{~m}, 3 \mathrm{H}), 1.86-1.77(\mathrm{~m}, 6 \mathrm{H}), 1.70-1.59(\mathrm{~m}, 6 \mathrm{H}) .{ }^{13} \mathrm{C} \mathrm{NMR}(101 \mathrm{MHz}$, Chloroform-d) $\delta$ 196.0, 50.5, 42.3, 36.0, 30.0, 28.9. HRMS (EI) $\mathrm{m} / \mathrm{z}$ calcd for $\mathrm{C}_{12} \mathrm{H}_{18} \mathrm{OS}_{2}\left[\mathrm{M}^{+}\right]$: 242.0799, found 242.0799.

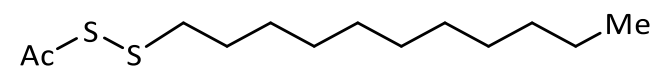

S1

SS-undecyl ethane(dithioperoxoate) (S1): colorless oil (29.4 mg, 56\% yield). ${ }^{1} \mathrm{H}$ NMR (400 MHz, Chloroform-d) $\delta$ $2.71(\mathrm{t}, J=6.7 \mathrm{~Hz}, 2 \mathrm{H}), 2.44(\mathrm{~s}, 3 \mathrm{H}), 1.62(\mathrm{p}, J=7.3 \mathrm{~Hz}, 2 \mathrm{H}), 1.39-1.31(\mathrm{~m}, 2 \mathrm{H}), 1.29-1.21(\mathrm{~m}, 14 \mathrm{H}), 0.88(\mathrm{t}, J=6.7$ $\mathrm{Hz}, 3 \mathrm{H}$ ). ${ }^{13} \mathrm{C}$ NMR (101 MHz, Chloroform-d) $\delta 195.4,39.1,32.1,29.7,29.7,29.6,29.5,29.3,29.2,29.1,28.6,22.8$, 14.3. HRMS (EI) $\mathrm{m} / \mathrm{z}$ calcd for $\mathrm{C}_{13} \mathrm{H}_{26} \mathrm{OS}_{2}\left[\mathrm{M}^{+}\right]$: 262.1425, found 262.1428 .<smiles>O=C(SSCc1ccccc1)c1ccccc1</smiles>

S3

SS-tert-butyl benzo(dithioperoxoate) (S3): white solid (18.6 mg, $41 \%$ yield). ${ }^{1} \mathrm{H}$ NMR (400 MHz, Chloroform-d) $\delta$ $8.05-8.02(\mathrm{~m}, 2 \mathrm{H}), 7.64-7.59(\mathrm{~m}, 1 \mathrm{H}), 7.51-7.46(\mathrm{~m}, 2 \mathrm{H}), 1.36(\mathrm{~s}, 9 \mathrm{H}) .{ }^{13} \mathrm{C}$ NMR (101 MHz, Chloroform-d) $\delta$ 190.5, 136.0, 134.1, 129.0, 127.9, 49.2, 29.9. HRMS (EI) m/z calcd for $\mathrm{C}_{11} \mathrm{H}_{14} \mathrm{OS}_{2}\left[\mathrm{M}^{+}\right]$: 226.0486, found 226.0488 . 


\section{Computational Details}

Optimized Gaussian Structures and CBS-QB3 Energies

Et•

CBS-QB3 Enthalpy $=-78.966621 \quad$ CBS-QB3 Free Energy $=-78.995756$

02

C $\quad 0.79410800 \quad 0.00000000 \quad-0.01959100$

$\begin{array}{llll}H & 1.35157000 & 0.92631300 & 0.04334300\end{array}$

$\begin{array}{llll}\text { H } & 1.35156300 & -0.92631900 & 0.04334100\end{array}$

C $\quad-0.69340300 \quad 0.00000200 \quad-0.00167300$

H $\quad-1.10717000-0.88612100-0.49272400$

H $\quad-1.09302900-0.00010200 \quad 1.02617600$

$\begin{array}{llll}H & -1.10716800 & 0.88621800 & -0.49255400\end{array}$

${ }^{t} \mathrm{Bu}_{\mathrm{S}^{-}}-\mathrm{S}_{-\mathrm{S}}-\mathrm{S}_{t_{\mathrm{Bu}}}$

CBS-QB3 Enthalpy $=-1906.015841 \quad$ CBS-QB3 Free Energy $=-1906.082022$

01

$\begin{array}{llll}\text { S } & 0.60321700 & 0.87865500 & -0.63221900\end{array}$

$\begin{array}{llll}\text { C } & 3.53286600 & -0.14933500 & 0.04123200\end{array}$

S $\quad-0.60321600-0.87864400-0.63223400$

$\begin{array}{llll}\text { S } & 2.04906200 & 0.69853300 & 0.83798000\end{array}$

S $\quad-2.04906400 \quad-0.69854600 \quad 0.83796500$

$\begin{array}{llll}\text { C } & -3.53286700 & 0.14933400 & 0.04122600\end{array}$

C $\quad 4.55803500 \quad-0.17989200 \quad 1.18608200$

$\begin{array}{llll}\text { H } & 4.19353200 & -0.76065500 & 2.03705700\end{array}$

$\begin{array}{llll}\text { H } & 5.48141700 & -0.64728300 & 0.82981100\end{array}$

$\begin{array}{llll}H & 4.80356400 & 0.82687600 & 1.53353300\end{array}$

C $\quad 3.17039100-1.56853700 \quad-0.40123700$

H $\quad 2.42312300-1.55930000-1.19691300$

$\begin{array}{llll}\text { H } & 4.06384700 & -2.07045500 & -0.78887600\end{array}$ 


\begin{tabular}{llll} 
H & 2.77670500 & -2.15773900 & 0.42920700 \\
C & 4.05187100 & 0.68403300 & -1.13271100 \\
H & 4.32853100 & 1.69070100 & -0.81215000 \\
H & 4.93752500 & 0.20392900 & -1.56320800 \\
H & 3.30039400 & 0.76822000 & -1.92052800 \\
C & -3.17038400 & 1.56853400 & -0.40124200 \\
H & -2.77669000 & 2.15773100 & 0.42920100 \\
H & -2.42311900 & 1.55929300 & -1.19692100 \\
H & -4.06383800 & 2.07045800 & -0.78887600 \\
C & -4.55802900 & 0.17989500 & 1.18608200 \\
H & -5.48140900 & 0.64729700 & 0.82981800 \\
H & -4.80356600 & -0.82687200 & 1.53352900 \\
H & -4.19351600 & 0.76065000 & 2.03705800 \\
C & -4.05188400 & -0.68402800 & -1.13271600 \\
H & -4.32854800 & -1.69069600 & -0.81215500 \\
\hline & -4.93753700 & -0.20391800 & -1.56320600 \\
\hline & -3.30041200 & -0.76821800 & -1.92053700
\end{tabular}

Dialkyl tetrasulfide 1 substitution by Et-radical<smiles>CC[SH](SCC(C)(C)C)SSCCC(C)C</smiles>

TS freq.: $-289.91 \mathrm{~cm}^{-1}$

CBS-QB3 Enthalpy $=-1984.981027 \quad$ CBS-QB3 Free Energy $=-1985.059267$

02

C $\quad-3.69611600-0.05682300-0.11501100$

S $\quad-2.23369800-1.12865100-0.63606100$

S $\quad-0.86903700 \quad-1.06819100 \quad 0.89224100$

S $\quad 0.60748300 \quad 0.64650400 \quad 0.51806900$

S $\quad 1.90762700 \quad-0.02833700-0.94292700$ 


\begin{tabular}{|c|c|c|c|}
\hline C & 3.31222800 & -0.93773700 & -0.07249900 \\
\hline C & 1.45621300 & 2.98728200 & 0.73820800 \\
\hline $\mathrm{H}$ & 2.47867100 & 2.71350000 & 0.97164000 \\
\hline $\mathrm{H}$ & 0.83739700 & 3.22674700 & 1.59668400 \\
\hline C & -4.67411600 & -0.20913800 & -1.28998800 \\
\hline $\mathrm{H}$ & -5.57797200 & 0.37409800 & -1.08724700 \\
\hline $\mathrm{H}$ & -4.23997900 & 0.15625300 & -2.22412800 \\
\hline $\mathrm{H}$ & -4.97166600 & -1.25123500 & -1.43211500 \\
\hline C & -4.30882600 & -0.59552900 & 1.17957100 \\
\hline $\mathrm{H}$ & -3.58970300 & -0.55770000 & 2.00040900 \\
\hline $\mathrm{H}$ & -5.17564300 & 0.01293000 & 1.46054400 \\
\hline $\mathrm{H}$ & -4.63935300 & -1.62968500 & 1.06025600 \\
\hline C & -3.25683400 & 1.40012700 & 0.04322600 \\
\hline $\mathrm{H}$ & -2.80992200 & 1.78265900 & -0.87681100 \\
\hline $\mathrm{H}$ & -4.12431100 & 2.02182700 & 0.29162300 \\
\hline $\mathrm{H}$ & -2.52660500 & 1.50609100 & 0.84789300 \\
\hline C & 2.78035200 & -2.17429000 & 0.65509000 \\
\hline $\mathrm{H}$ & 3.61760400 & -2.73305100 & 1.08830200 \\
\hline $\mathrm{H}$ & 2.10619900 & -1.89792000 & 1.46762300 \\
\hline $\mathrm{H}$ & 2.23812300 & -2.83434400 & -0.02435200 \\
\hline C & 4.22537200 & -1.34328100 & -1.24033800 \\
\hline $\mathrm{H}$ & 4.59618100 & -0.47052400 & -1.78399500 \\
\hline $\mathrm{H}$ & 5.08980300 & -1.88949200 & -0.84971400 \\
\hline $\mathrm{H}$ & 3.70684600 & -1.99607800 & -1.94697400 \\
\hline C & 4.04433600 & -0.00306500 & 0.89205700 \\
\hline $\mathrm{H}$ & 4.87769400 & -0.53632300 & 1.36265700 \\
\hline $\mathrm{H}$ & 4.44790000 & 0.86837300 & 0.37129000 \\
\hline $\mathrm{H}$ & 3.37844000 & 0.34189500 & 1.68596100 \\
\hline C & 1.17680800 & 3.67350500 & -0.55556700 \\
\hline
\end{tabular}




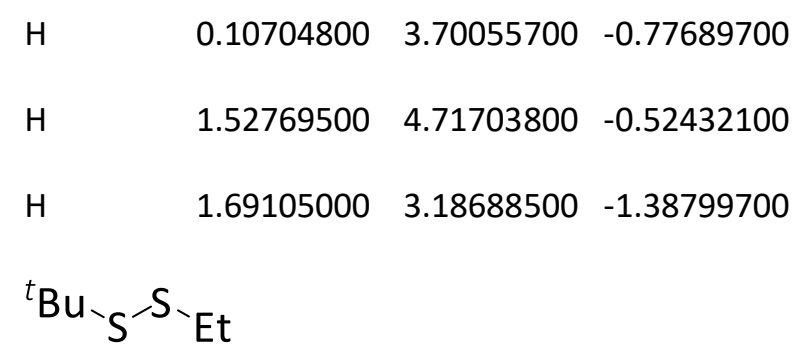




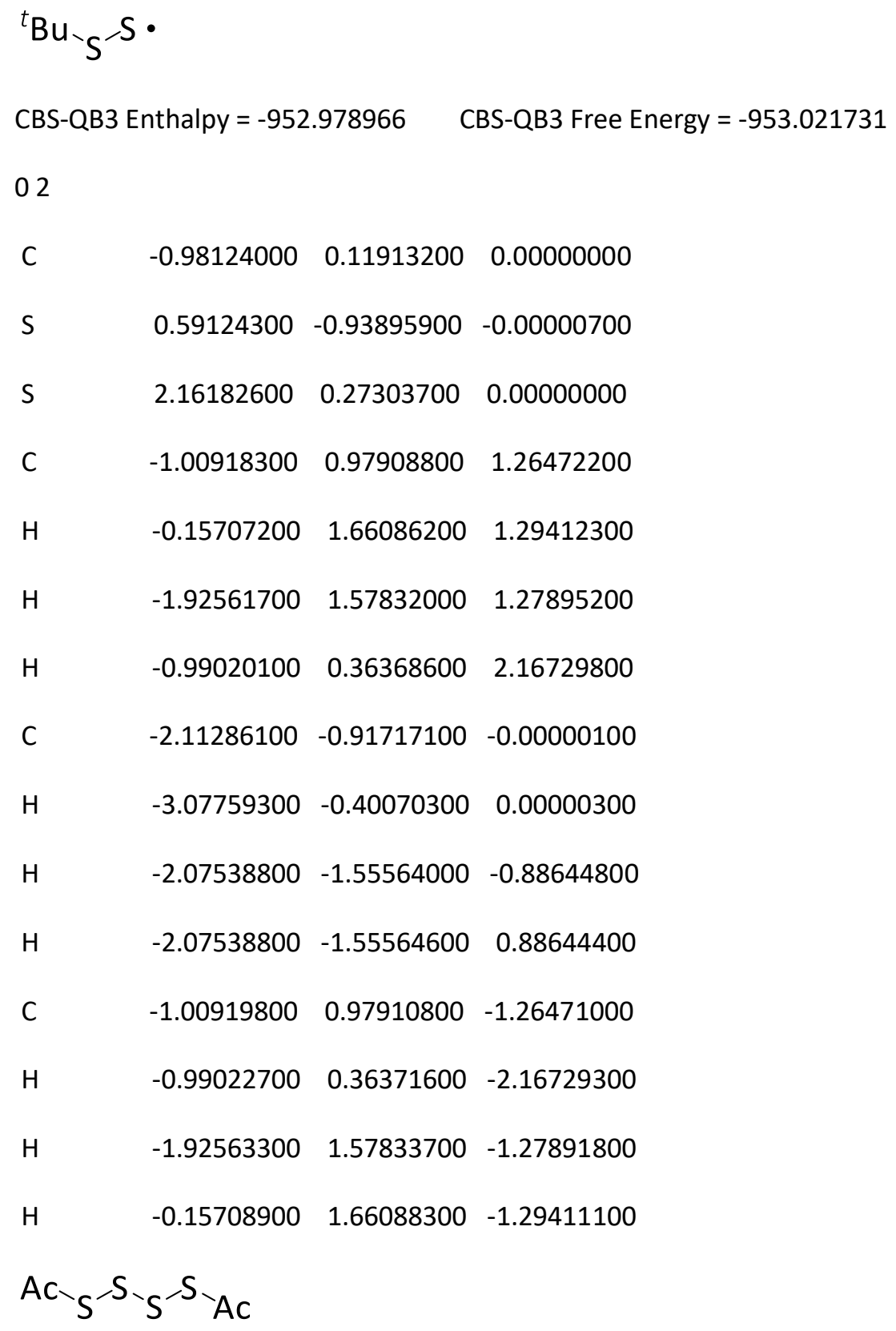




$$
\begin{array}{llll}
\text { H } & 2.74328100 & 1.50074100 & -1.22783200 \\
\text { C } & -4.60402500 & -0.71564000 & 0.19887200 \\
\text { H } & -5.13711200 & -0.56673900 & 1.13821500 \\
\text { H } & -5.22349000 & -0.34011400 & -0.62101800 \\
\text { H } & -4.42342200 & -1.77849600 & 0.02947000 \\
\text { C } & -3.32027500 & 0.08115400 & 0.22994900 \\
\text { C } & 3.49469500 & -0.17448500 & -0.12544400 \\
\text { O } & 4.41712600 & -0.91589100 & -0.30994800 \\
\text { O } & -3.13213800 & 1.07275700 & 0.86544400 \\
\text { Di acetyl tetrasulfide } 51 \text { substitution by Et-radical }
\end{array}
$$

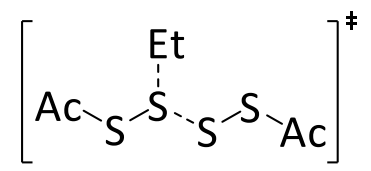

TS freq.: $-264.97 \mathrm{~cm}^{-1}$

CBS-QB3 Enthalpy $=-1975.995562$ CBS-QB3 Free Energy $=-1976.067493$

02

$\begin{array}{llll}\mathrm{S} & 2.42120900 & -1.17687200 & 0.53810100\end{array}$

S $\quad 0.91779900-1.16115000-0.87328300$

S $\quad-0.45561700 \quad 0.53021700 \quad-0.36015800$

S $\quad-1.81521300 \quad-0.31652000 \quad 0.96805000$

C $\quad-1.61749000 \quad 2.67739700 \quad-0.59428900$

$\begin{array}{llll}\text { H } & -2.56062000 & 2.23820800 & -0.89944700\end{array}$

$\begin{array}{llll}\text { H } & -0.99011300 & 3.01877000 & -1.41089800\end{array}$

$\begin{array}{lllll}\text { C } & 4.85032900 & 0.16508600 & 0.74522200\end{array}$

$\begin{array}{llll}\text { H } & 5.58507700 & -0.45015800 & 0.21739400\end{array}$

$\begin{array}{llll}\text { H } & 5.22515400 & 1.18814900 & 0.79360500\end{array}$

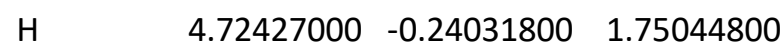

$\begin{array}{llll}\text { C } & -4.18380400 & -1.72572300 & 0.62992600\end{array}$

$\begin{array}{llll}\text { H } & -5.15030300 & -1.60598800 & 0.13893700\end{array}$ 


\begin{tabular}{|c|c|c|c|}
\hline $\mathrm{H}$ & -3.90947300 & -2.78449900 & 0.60381800 \\
\hline $\mathrm{H}$ & -4.24747600 & -1.41463600 & 1.67386700 \\
\hline C & -1.53679900 & 3.40034700 & 0.70695000 \\
\hline $\mathrm{H}$ & -0.50202200 & 3.58739000 & 1.00395900 \\
\hline $\mathrm{H}$ & -2.03077400 & 4.38155400 & 0.62600500 \\
\hline $\mathrm{H}$ & -2.03891300 & 2.85494200 & 1.50936600 \\
\hline C & 3.56102200 & 0.14841500 & -0.04699100 \\
\hline C & -3.13897600 & -0.94927900 & -0.14185500 \\
\hline 0 & 3.29785300 & 0.88980300 & -0.94626400 \\
\hline $\mathrm{O}$ & -3.14802200 & -0.77501400 & -1.32426000 \\
\hline
\end{tabular}

CBS-QB3 Enthalpy $=-1027.541604 \quad$ CBS-QB3 Free Energy $=-1027.584923$

01

C $\quad 2.35661900-0.62061300-0.00000900$

H $\quad 2.29171400-1.25139700-0.88827100$

H $\quad 2.29173700-1.25139600 \quad 0.88825500$

S $\quad 0.94957200 \quad 0.56440200 \quad 0.00000800$

S $\quad-0.61122400-0.87435900 \quad 0.00003200$

C $\quad-3.34781300 \quad-0.43379800 \quad-0.00004400$

$\begin{array}{llll}H & -3.44539800 & -1.06842700 & 0.88386400\end{array}$

$\begin{array}{llll}H & -4.13562400 & 0.31890100 & 0.00007600\end{array}$

H $\quad-3.44544500-1.06818400 \quad-0.88412300$

$\begin{array}{lllll}\text { C } & 3.65812100 & 0.18123900 & -0.00002600\end{array}$

$\begin{array}{llll}H & 4.51074700 & -0.50294500 & -0.00003600\end{array}$

$\begin{array}{llll}H & 3.73547200 & 0.81621300 & -0.88561900\end{array}$

$\begin{array}{llll}\text { H } & 3.73549500 & 0.81621400 & 0.88556400\end{array}$

$\begin{array}{llll}\text { C } & -2.00915400 & 0.27545300 & 0.00000400\end{array}$

$\begin{array}{llll}0 & -1.86236200 & 1.46708000 & 0.00001400\end{array}$ 


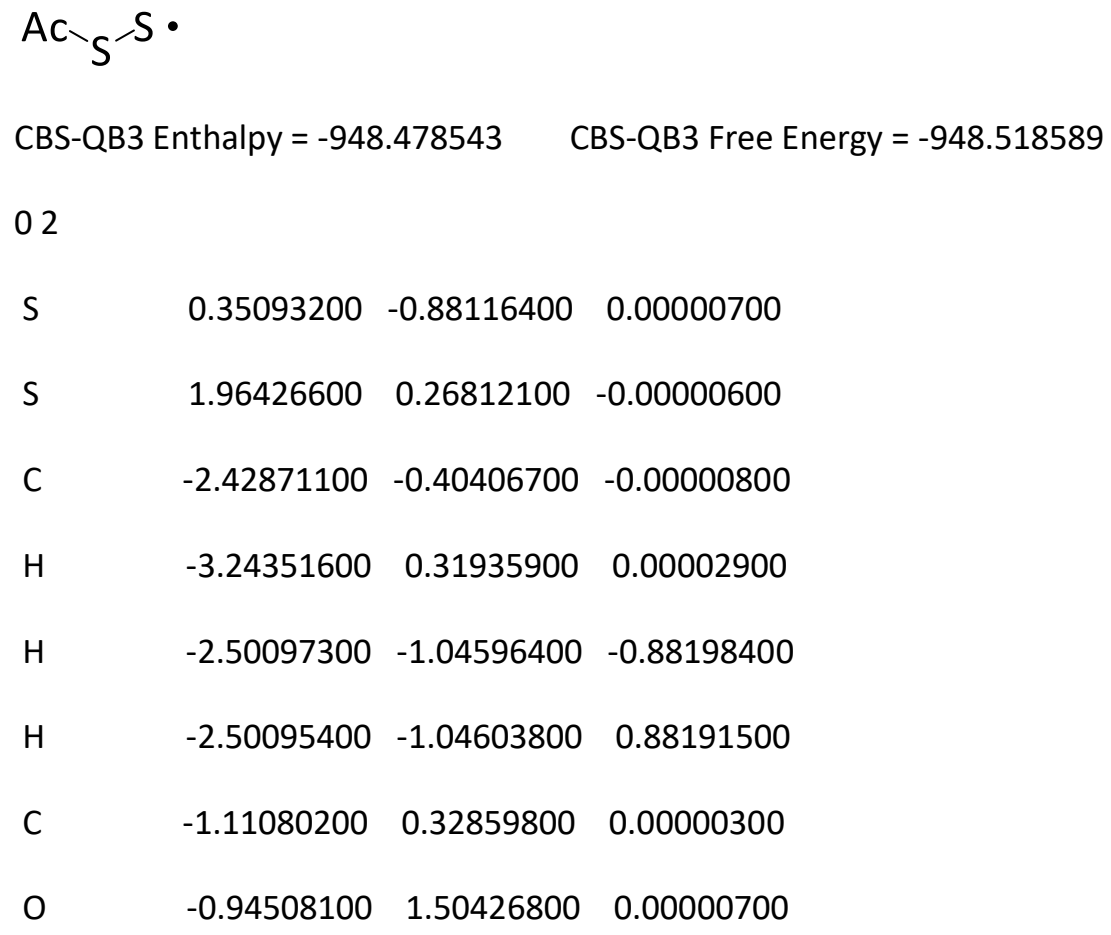




\section{References}

1. Hanss, D.; Freys, J. C.; Bernardinelli, G.; Wenger, O. S. Eur. J. Inorg. Chem. 2009, 4850.

2. Barton, D. H. R.; Crich, D.; Motherwell, W. B. J. Chem. Soc., Chem. Commun. 1983, 939.

3. (a) Patra, T.; Mukherjee, S.; Ma, J.; Strieth-Kalthoff, F.; Glorius, F. Angew. Chem. Int. Ed. 2019, 58, 10514. (b) Soni, V. K.; Lee, S.; Kang, J.; Moon, Y. K.; Hwang, H. S.; You, Y.; Cho, E. J. ACS Catal. 2019, 9, 10454. (c) Patra, T.; Bellotti, P.; Strieth-Kalthoff, F.; Glorius. F. Angew. Chem. Int. Ed. 2020, 59, 3172.

4. For the synthesis of dialkyl tetrasulfides: (a) Chauvin, J.-P. R.; Griesser, M.; Pratt, D. A. Chem. Sci. 2019, 10, 4999. (b) Cerda, M. M.; Hammers, M.D.; Earp, M.S.; Zakharov, L.N.; Pluth, M.D. Org. Lett. 2017, 19, 2314. (c) Derbesy, G.; Harpp, D. N. Tetrahedron Lett. 1994, 35, 5381. For diacyltetrasulfides: (d) Rao, M. V.; Reese, C. B.; Zhao, Z. Tetrahedron Lett. 1992, 33, 4839. 
8. NMR Spectra

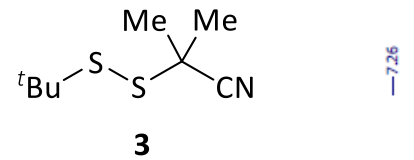

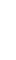

${ }^{1} \mathrm{H}$ NMR (400 MHz, $\mathrm{CDCl}_{3}$ )
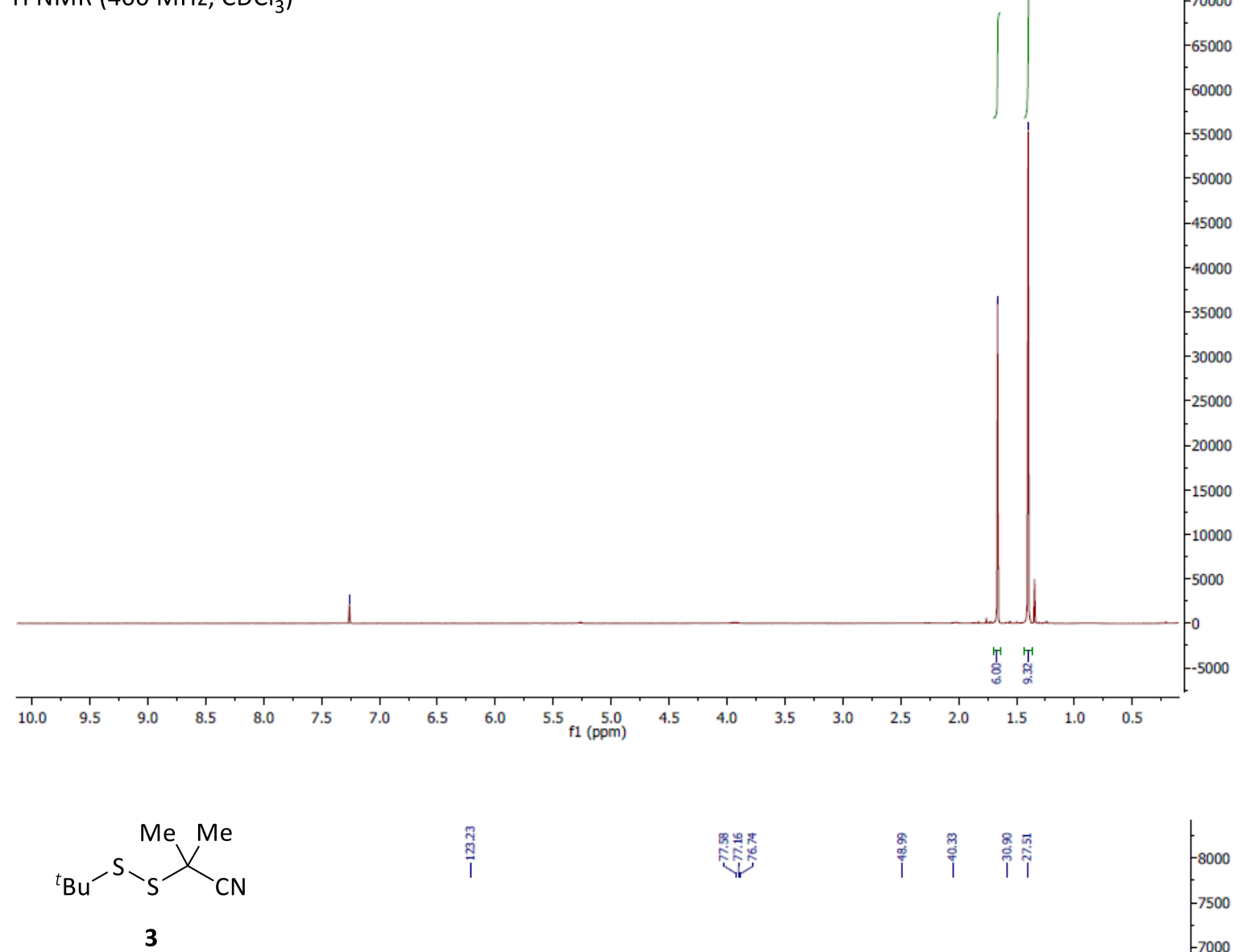

3

${ }^{13} \mathrm{C} \mathrm{NMR}\left(101 \mathrm{MHz}, \mathrm{CDCl}_{3}\right)$

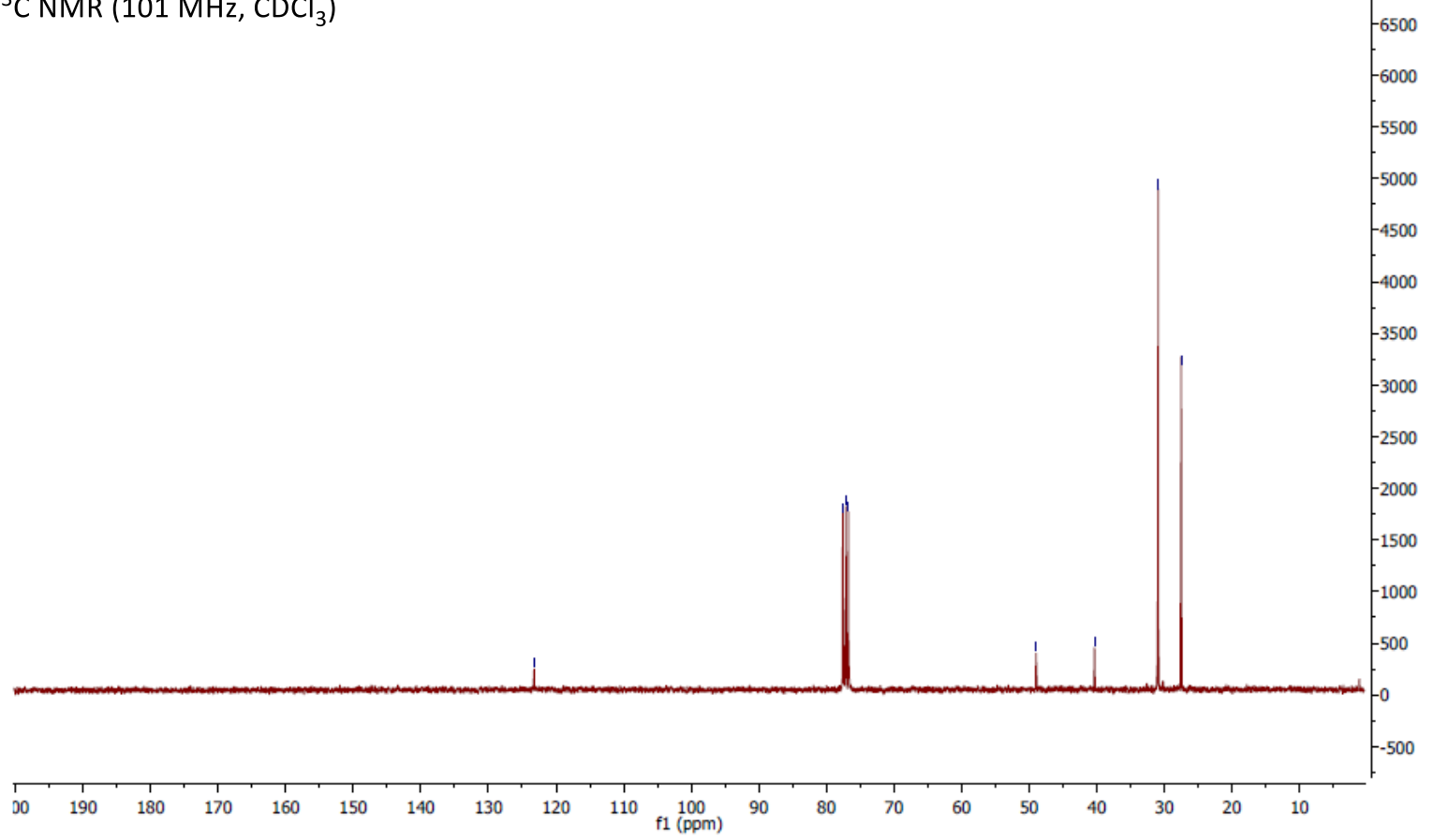



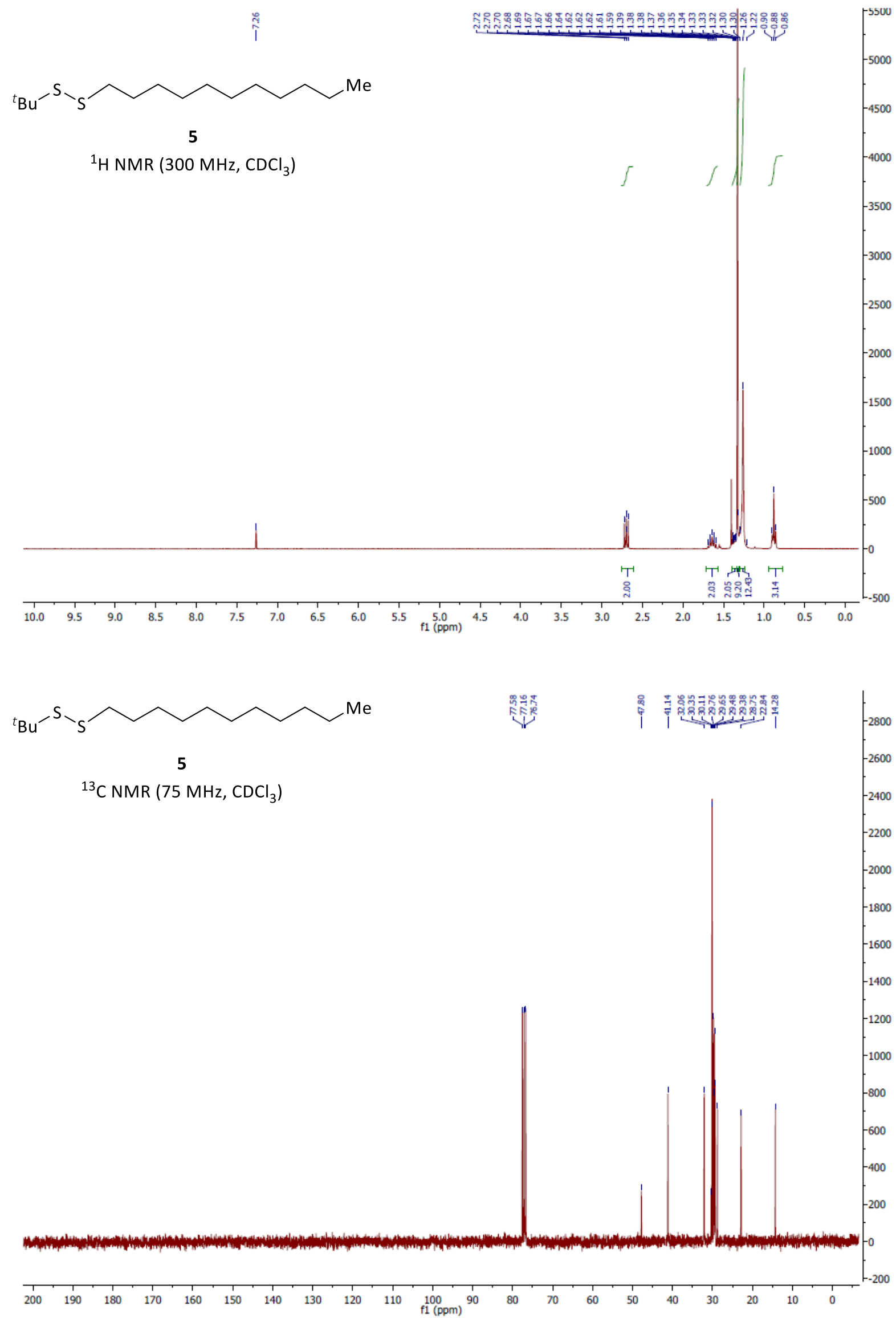

${ }^{13} \mathrm{C} \mathrm{NMR}\left(75 \mathrm{MHz}, \mathrm{CDCl}_{3}\right.$ ) 

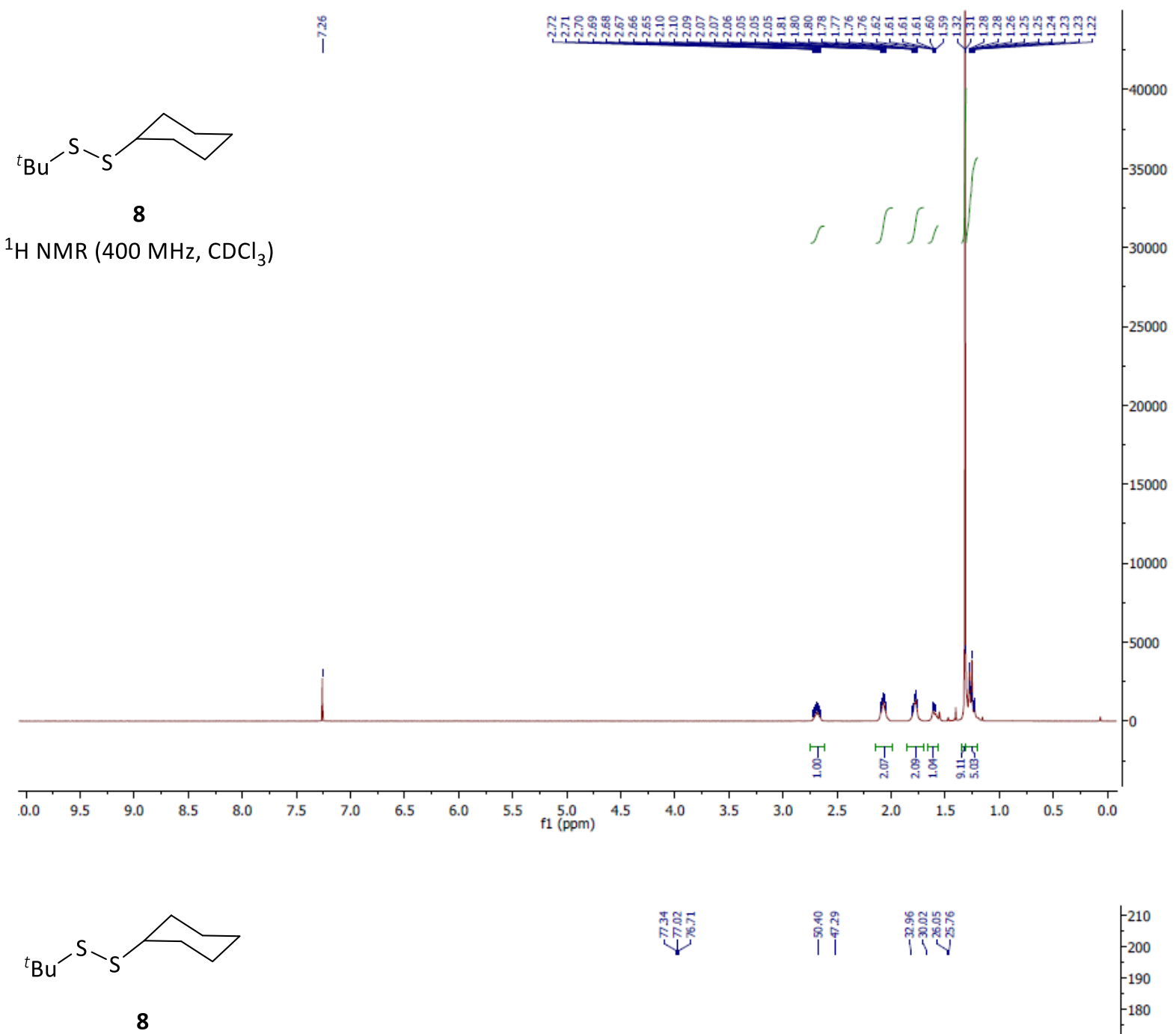

${ }^{13} \mathrm{C}$ NMR $\left(101 \mathrm{MHz}, \mathrm{CDCl}_{3}\right)$

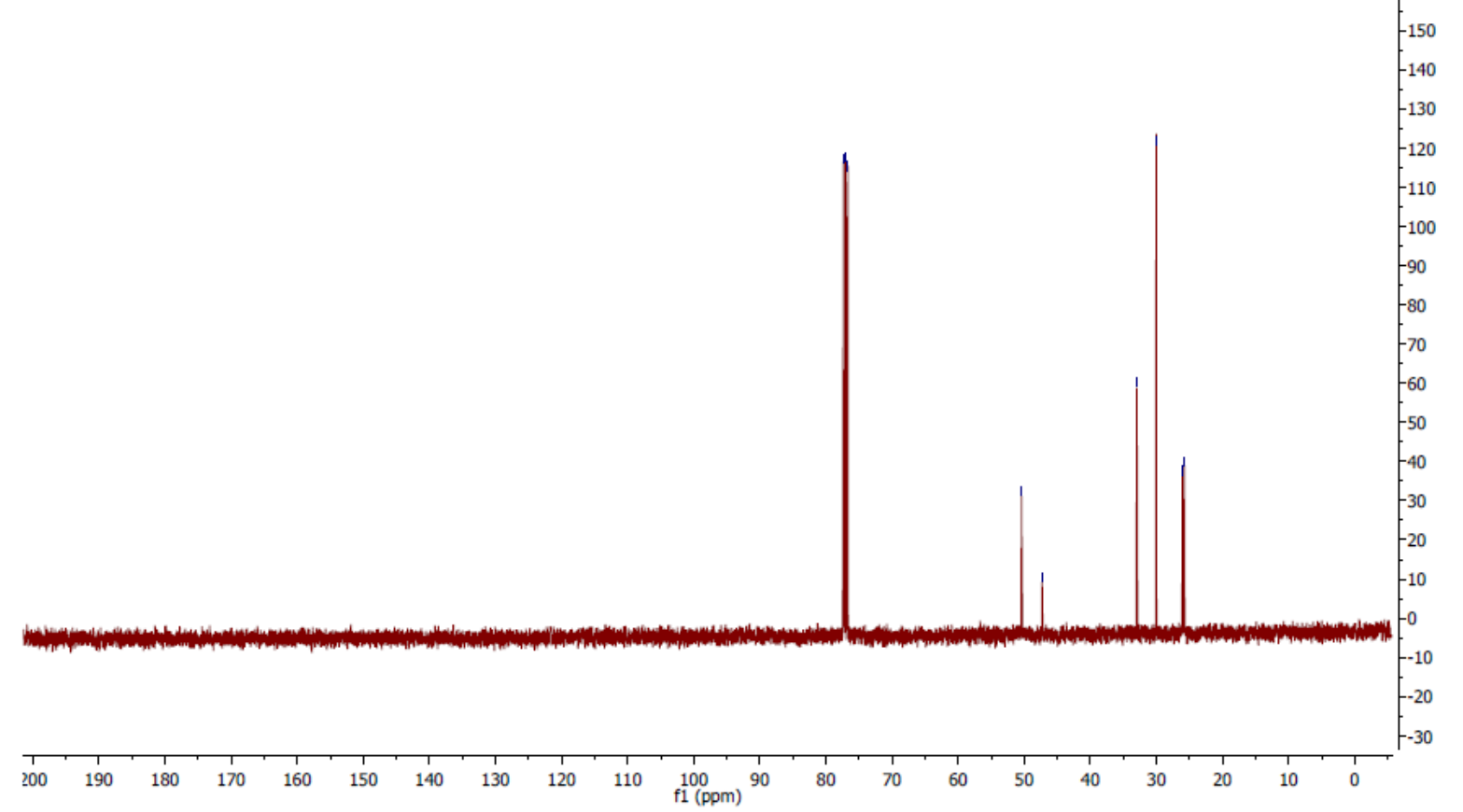



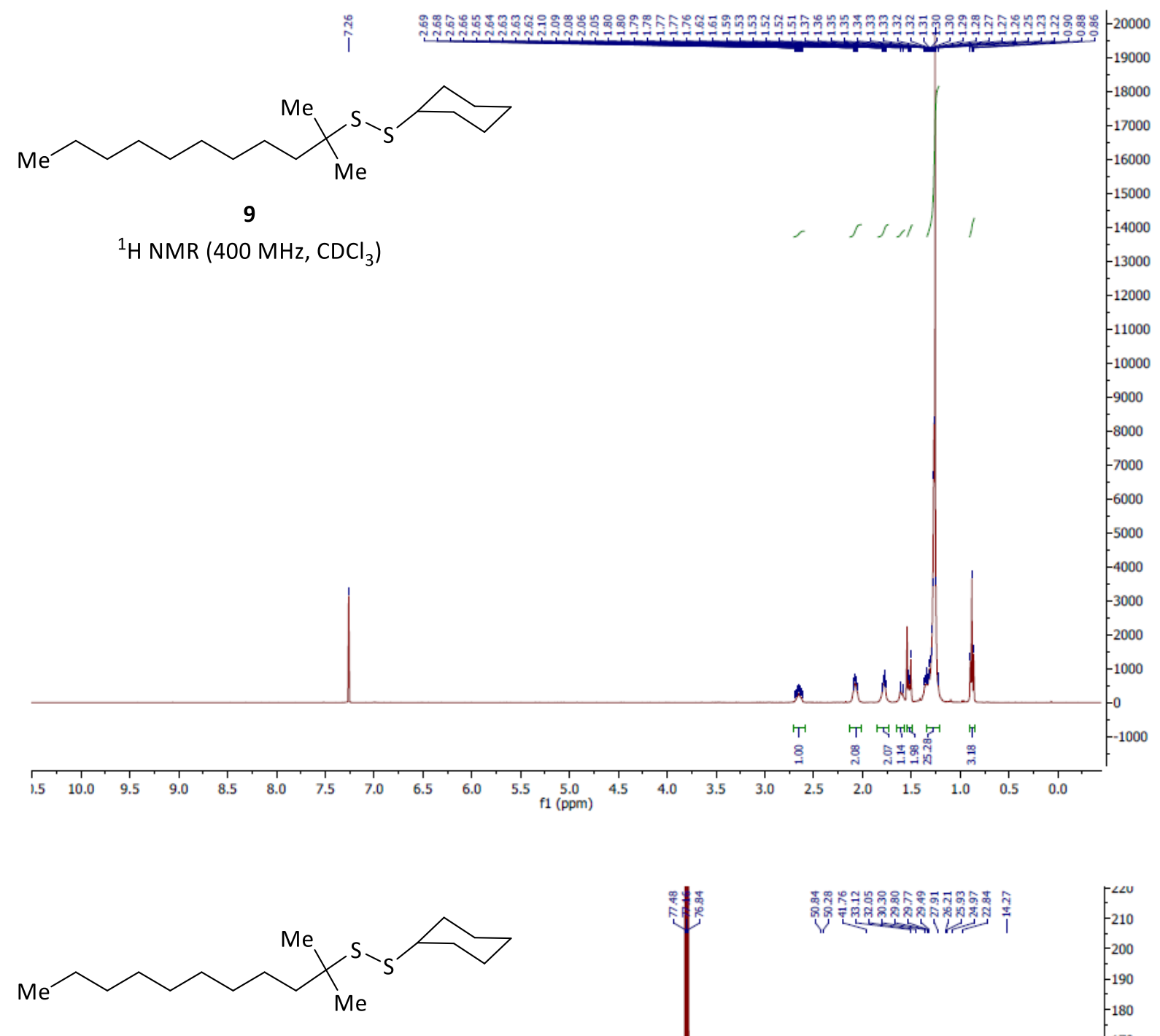

9

${ }^{13} \mathrm{C}$ NMR (101 MHz, CDCl 3 )

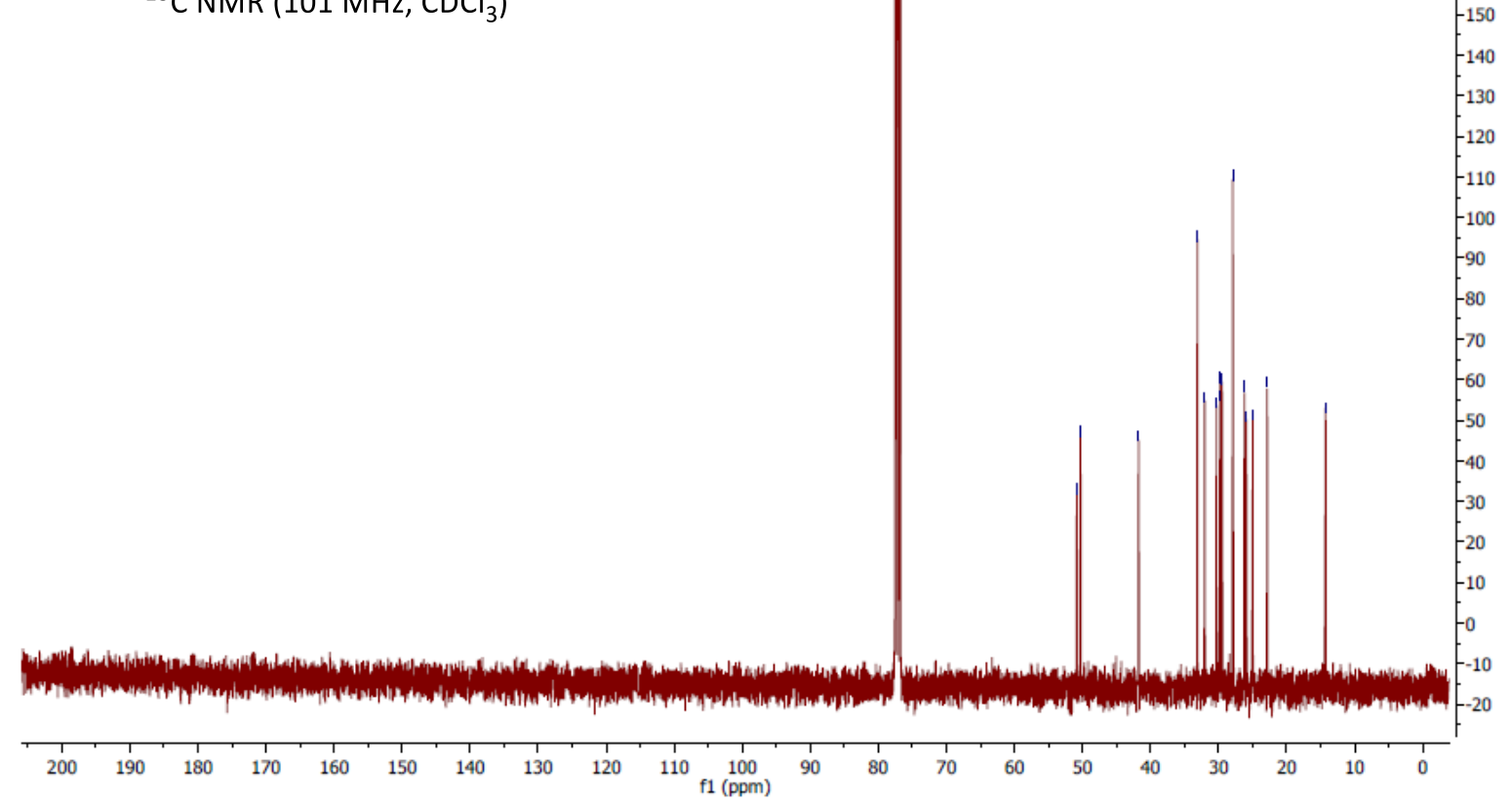



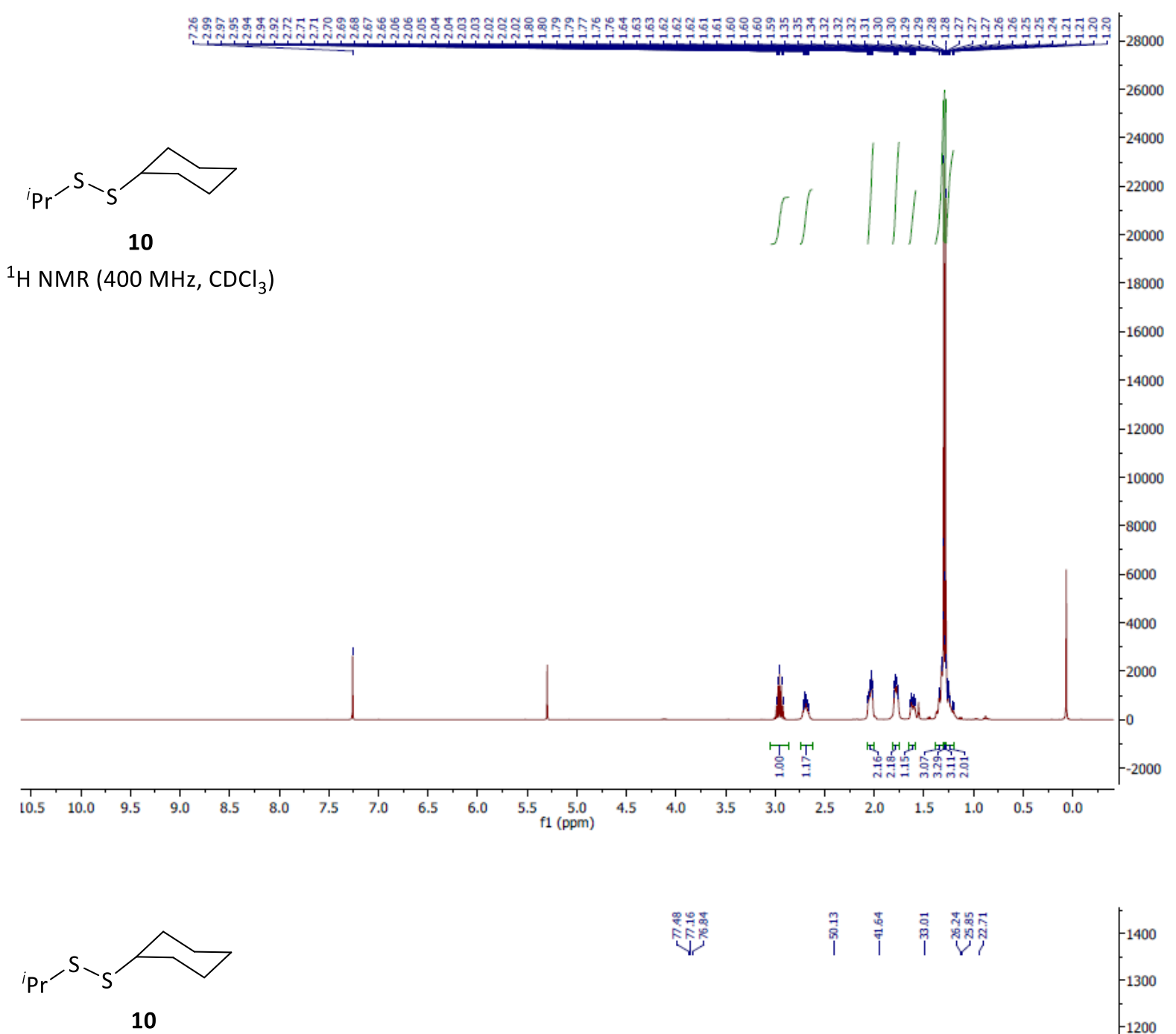

${ }^{13} \mathrm{C}$ NMR (101 MHz, $\mathrm{CDCl}_{3}$ )

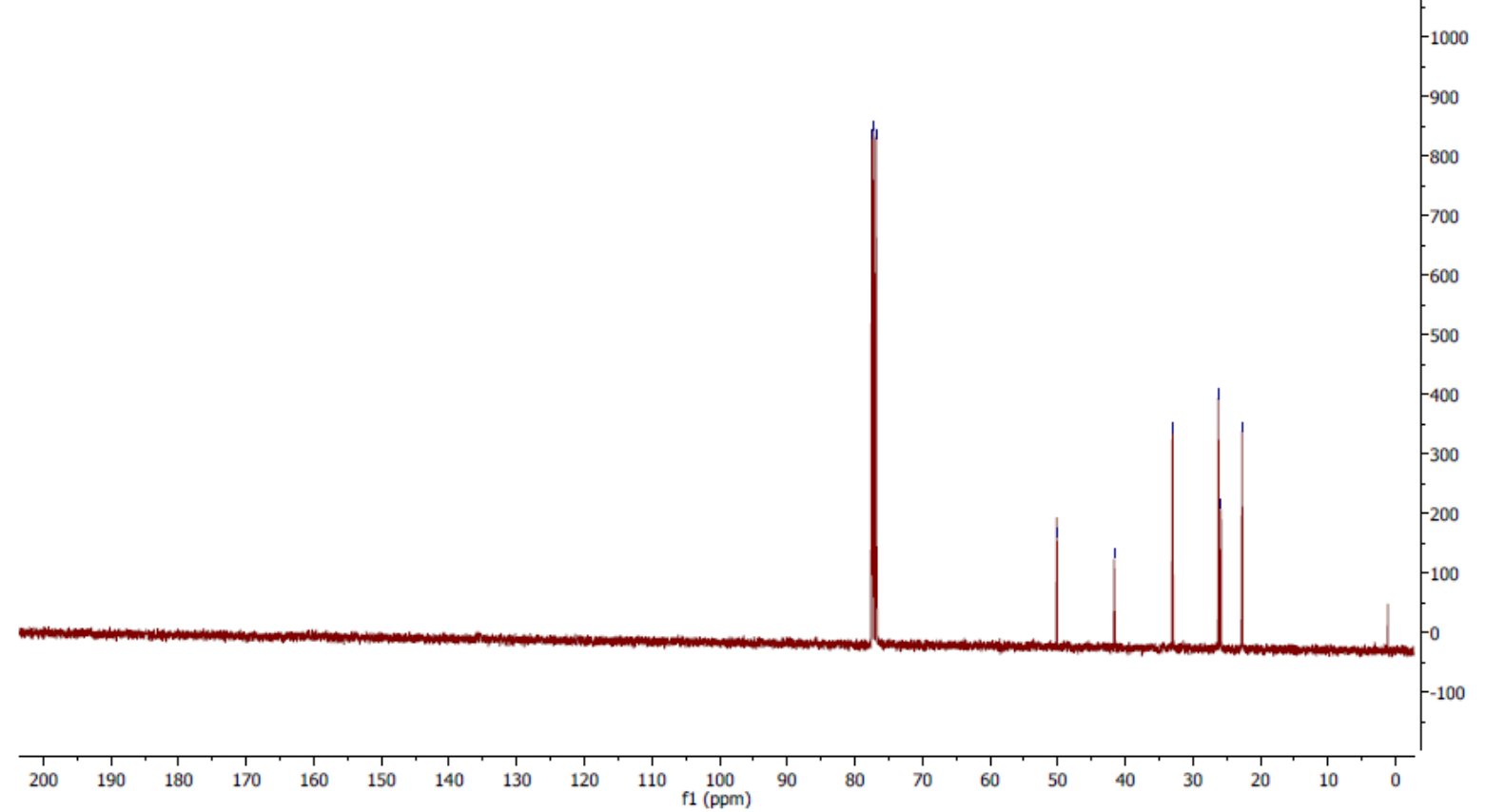




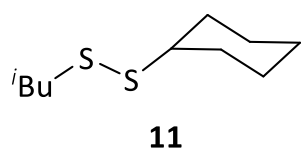

11

${ }^{1} \mathrm{H}$ NMR $\left(400 \mathrm{MHz}, \mathrm{CDCl}_{3}\right)$
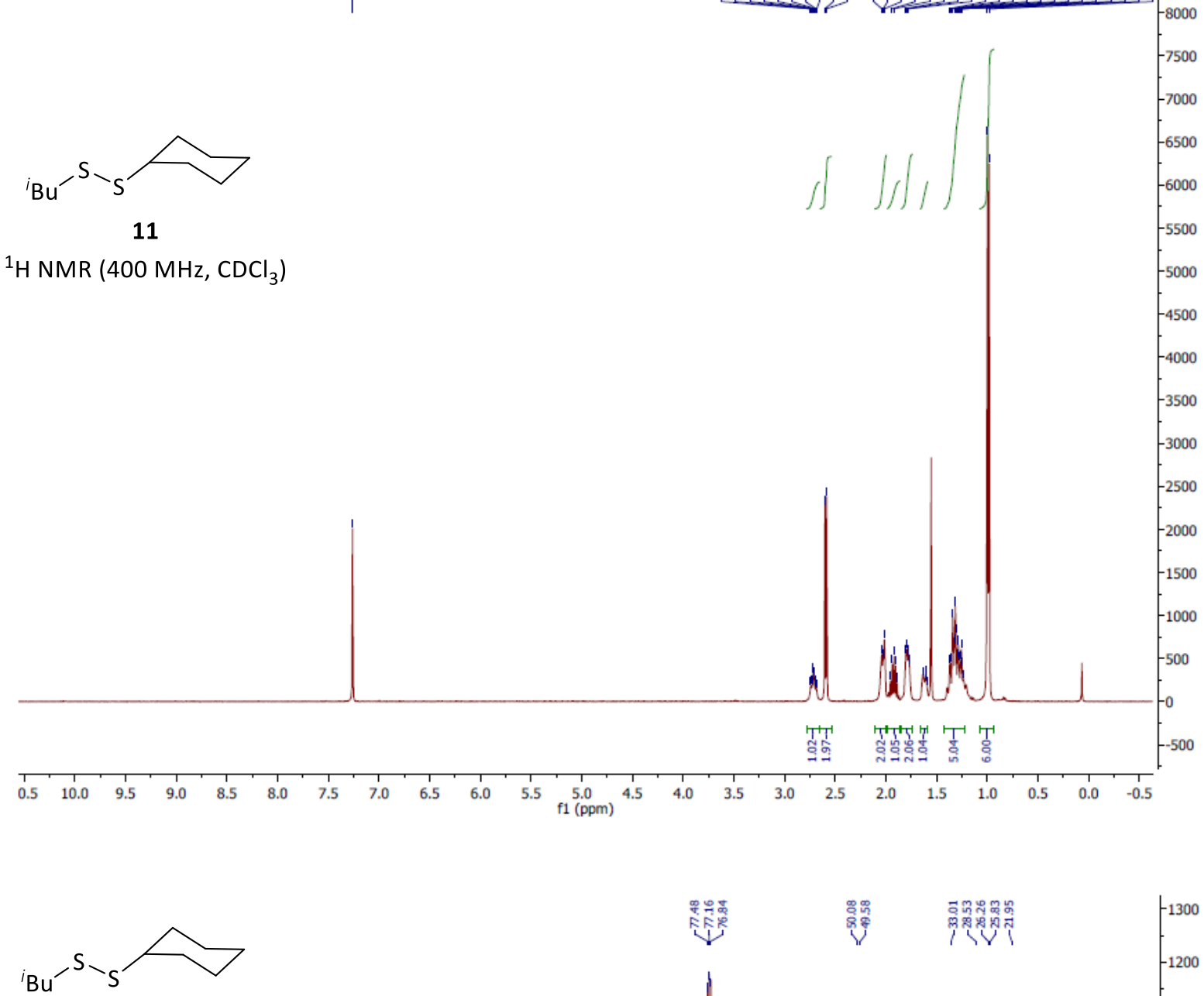

11

${ }^{13} \mathrm{C}$ NMR (101 MHz, $\mathrm{CDCl}_{3}$ )

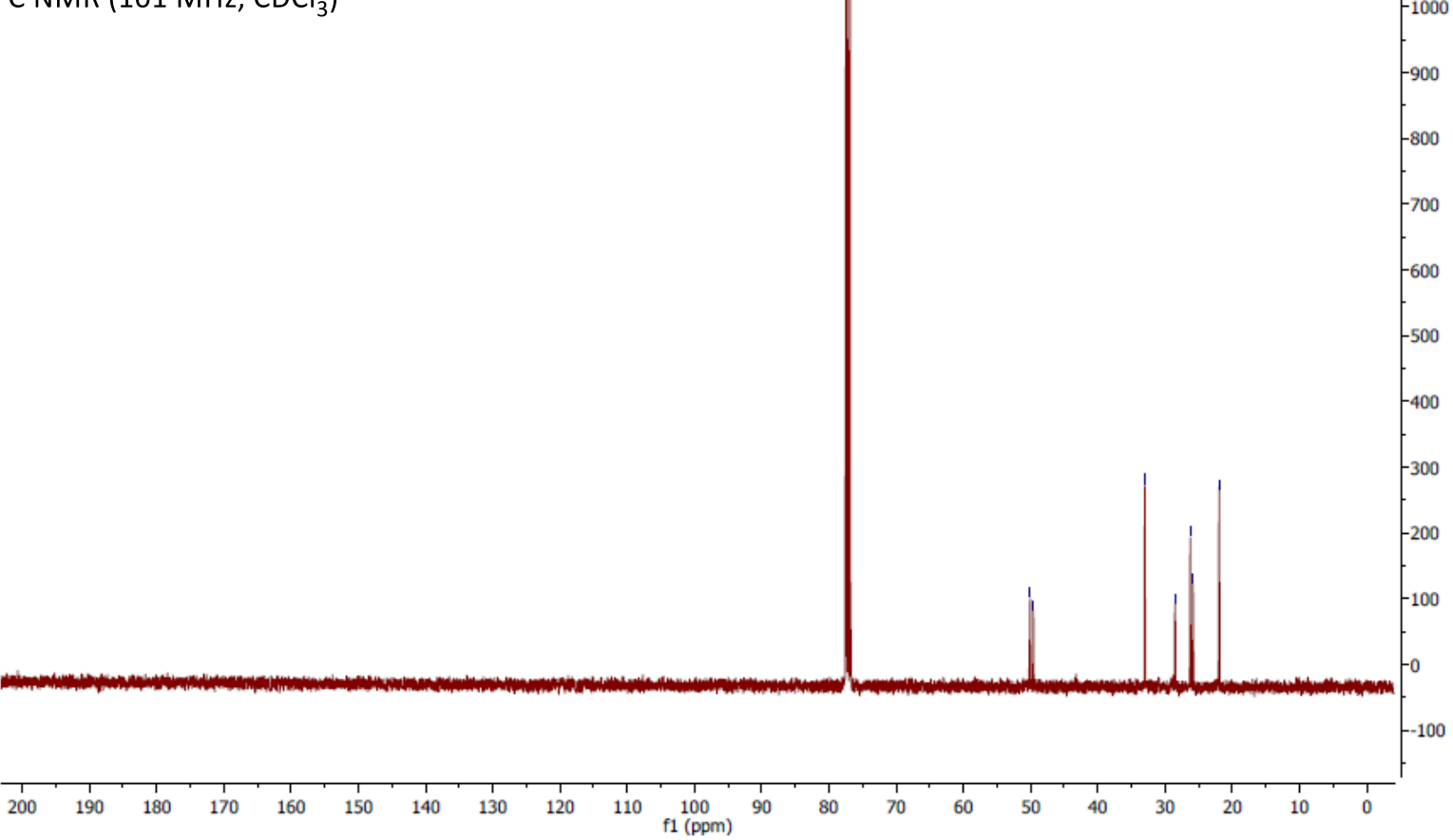




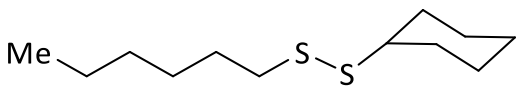

12

${ }^{1} \mathrm{H} \mathrm{NMR}\left(400 \mathrm{MHz}, \mathrm{CDCl}_{3}\right)$
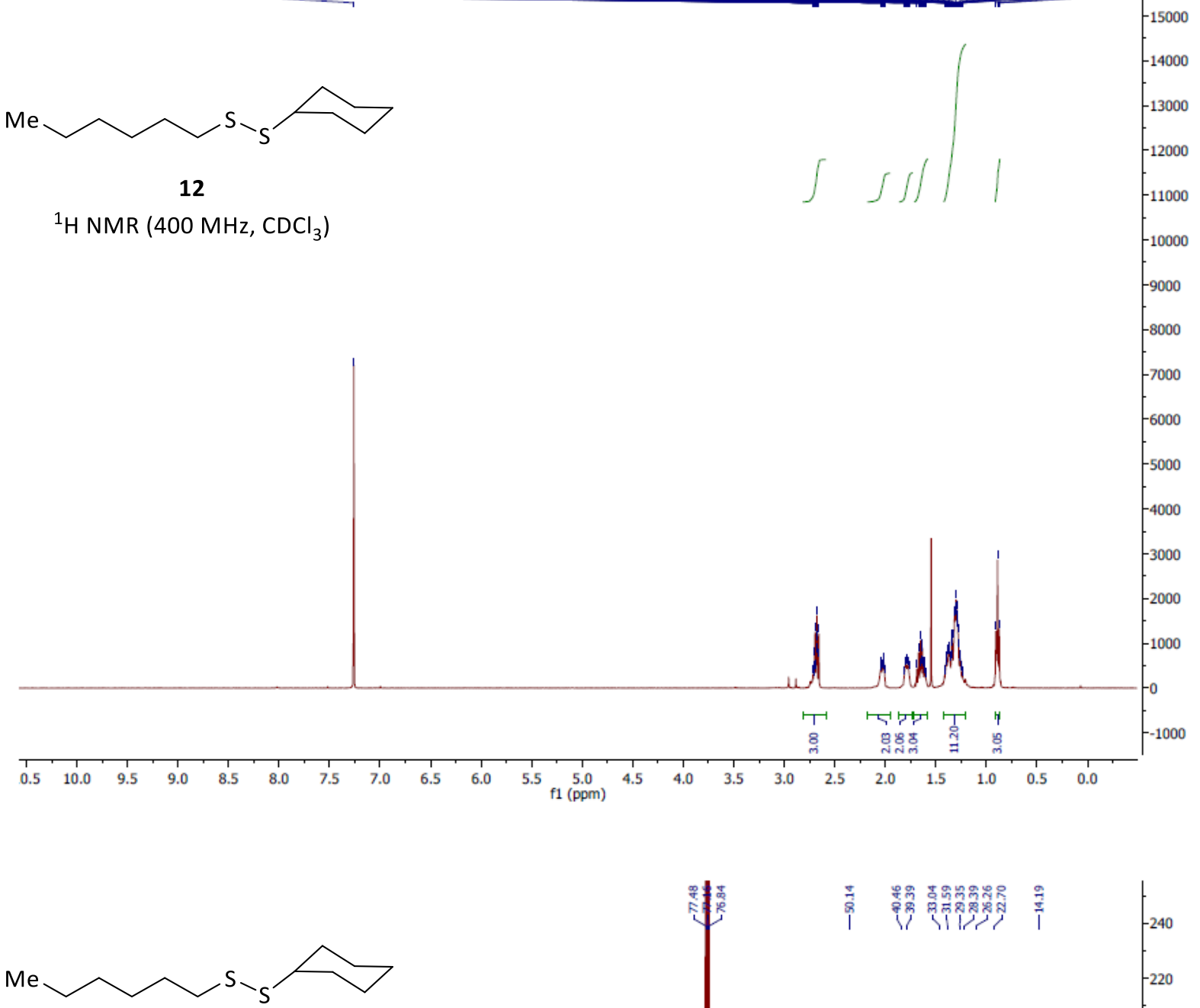

12

${ }^{13} \mathrm{C}$ NMR $\left(101 \mathrm{MHz}, \mathrm{CDCl}_{3}\right)$

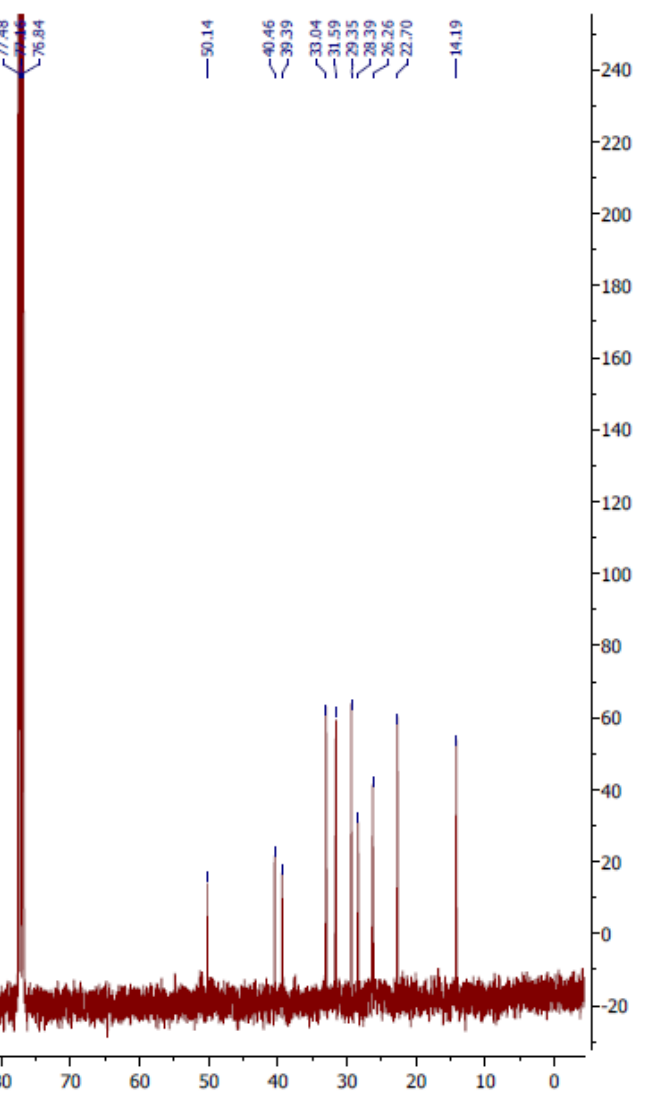



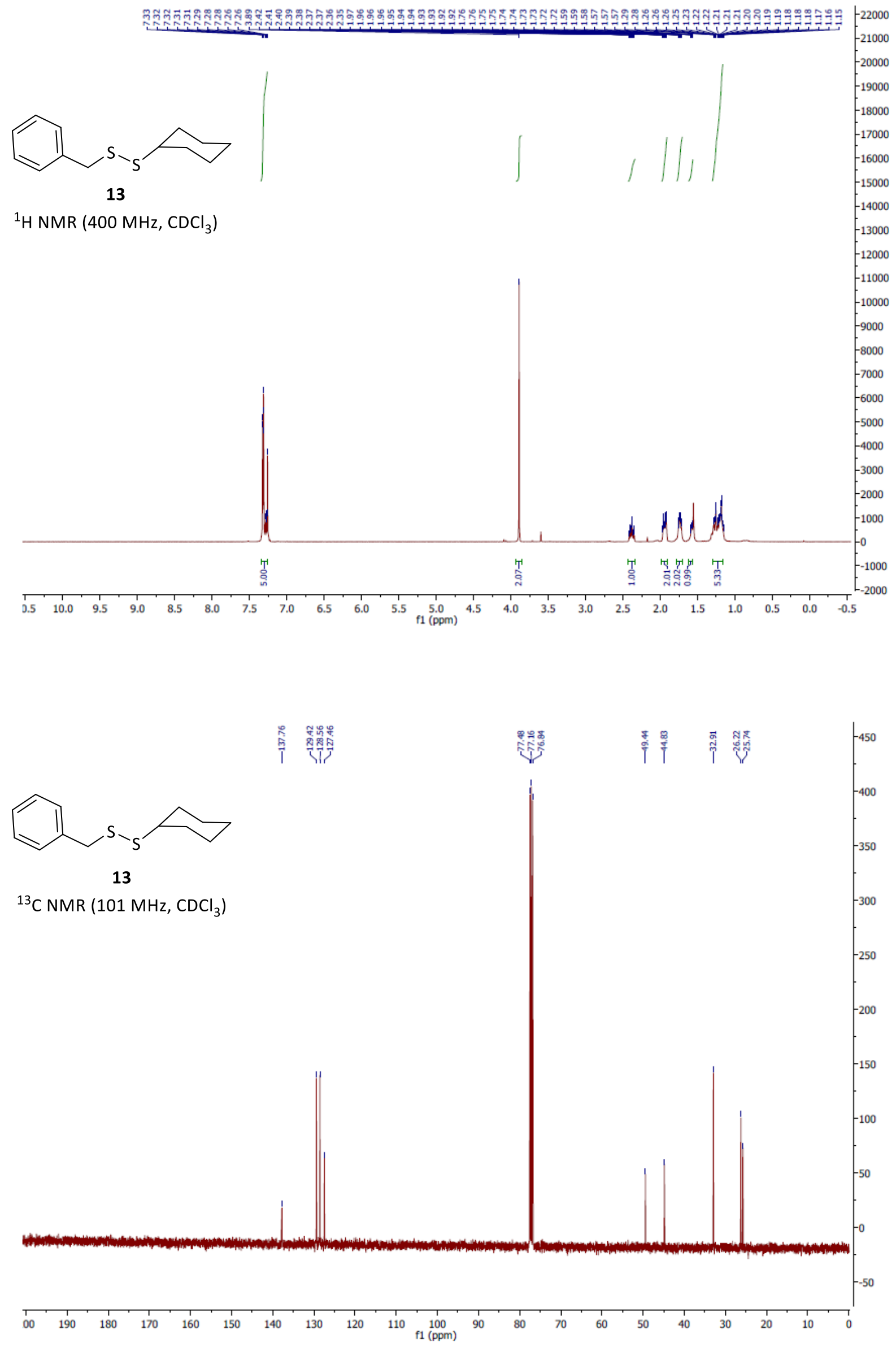

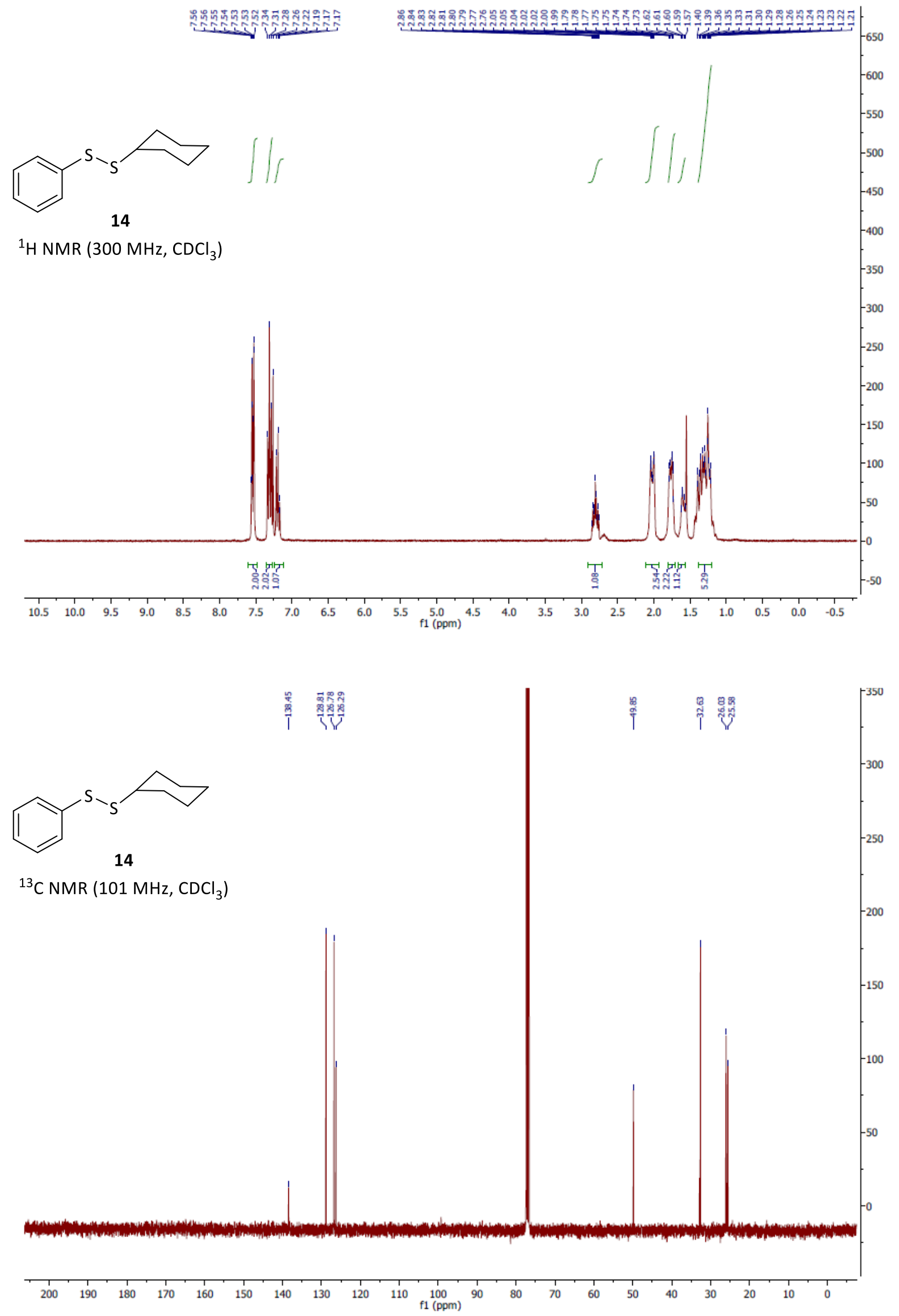

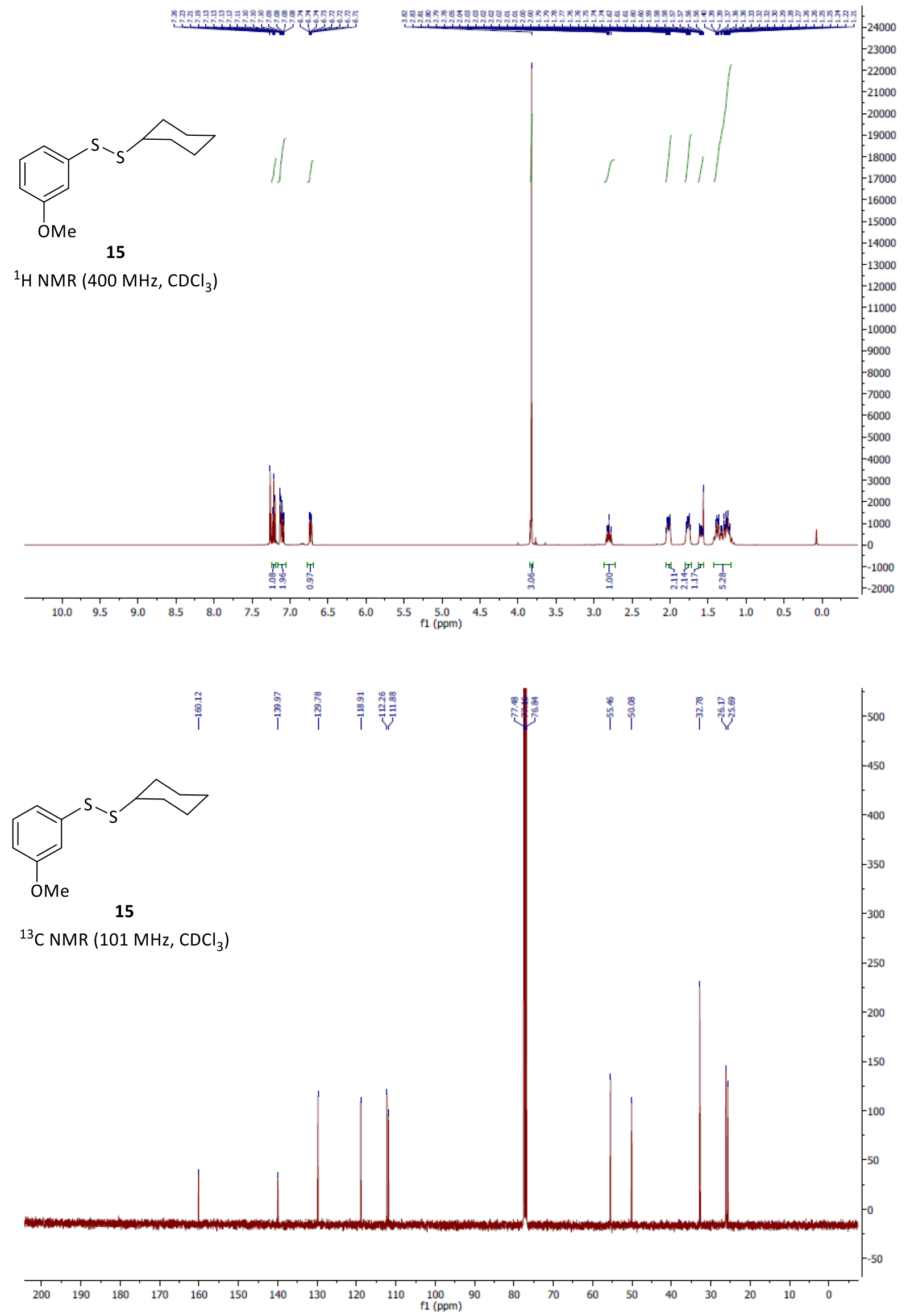

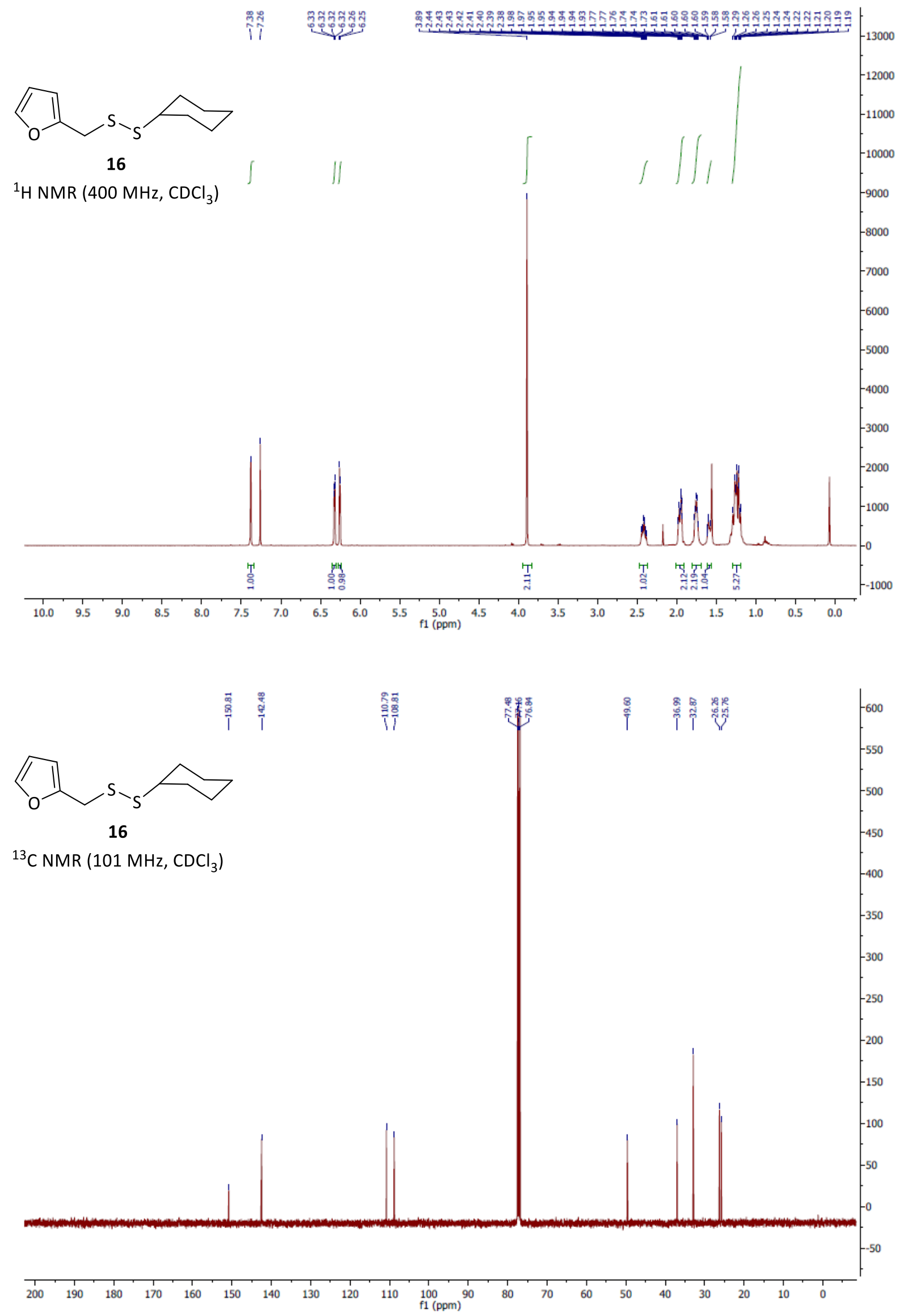

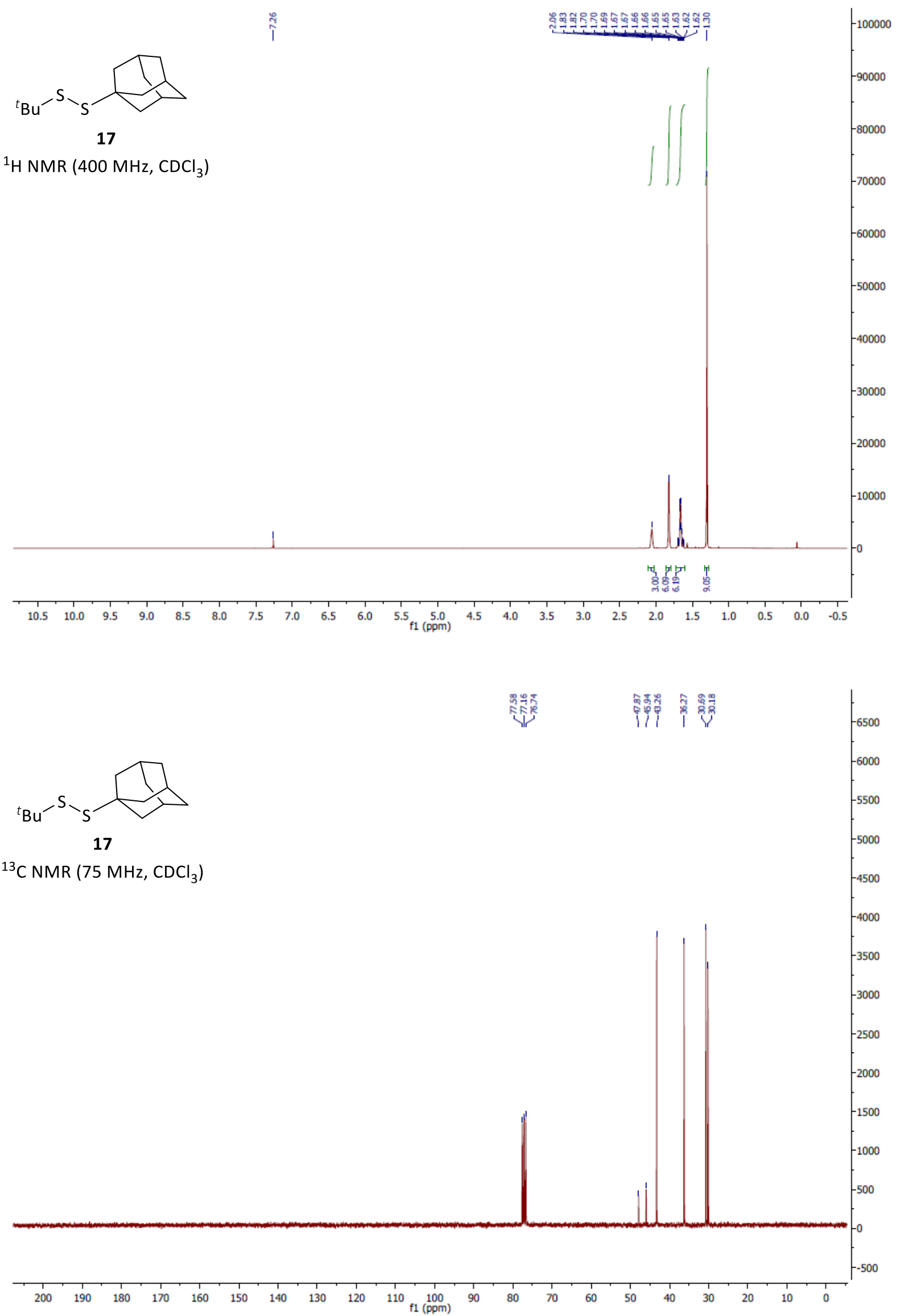

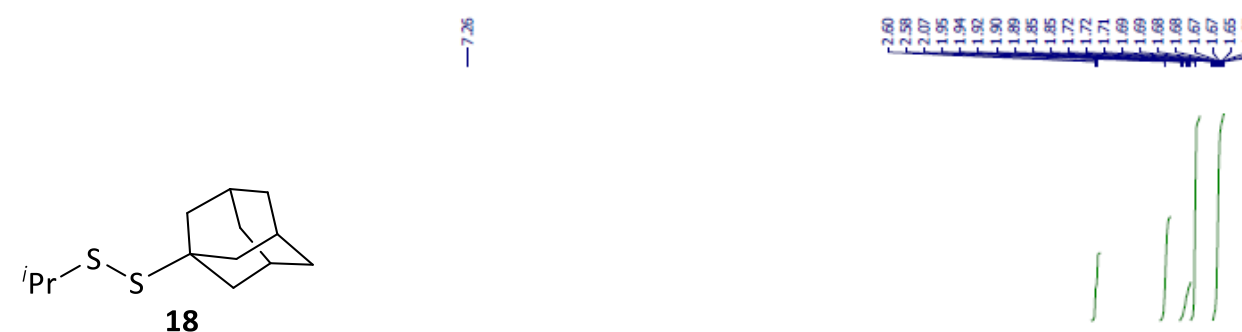

${ }^{1} \mathrm{H}$ NMR (400 MHz, $\mathrm{CDCl}_{3}$ )
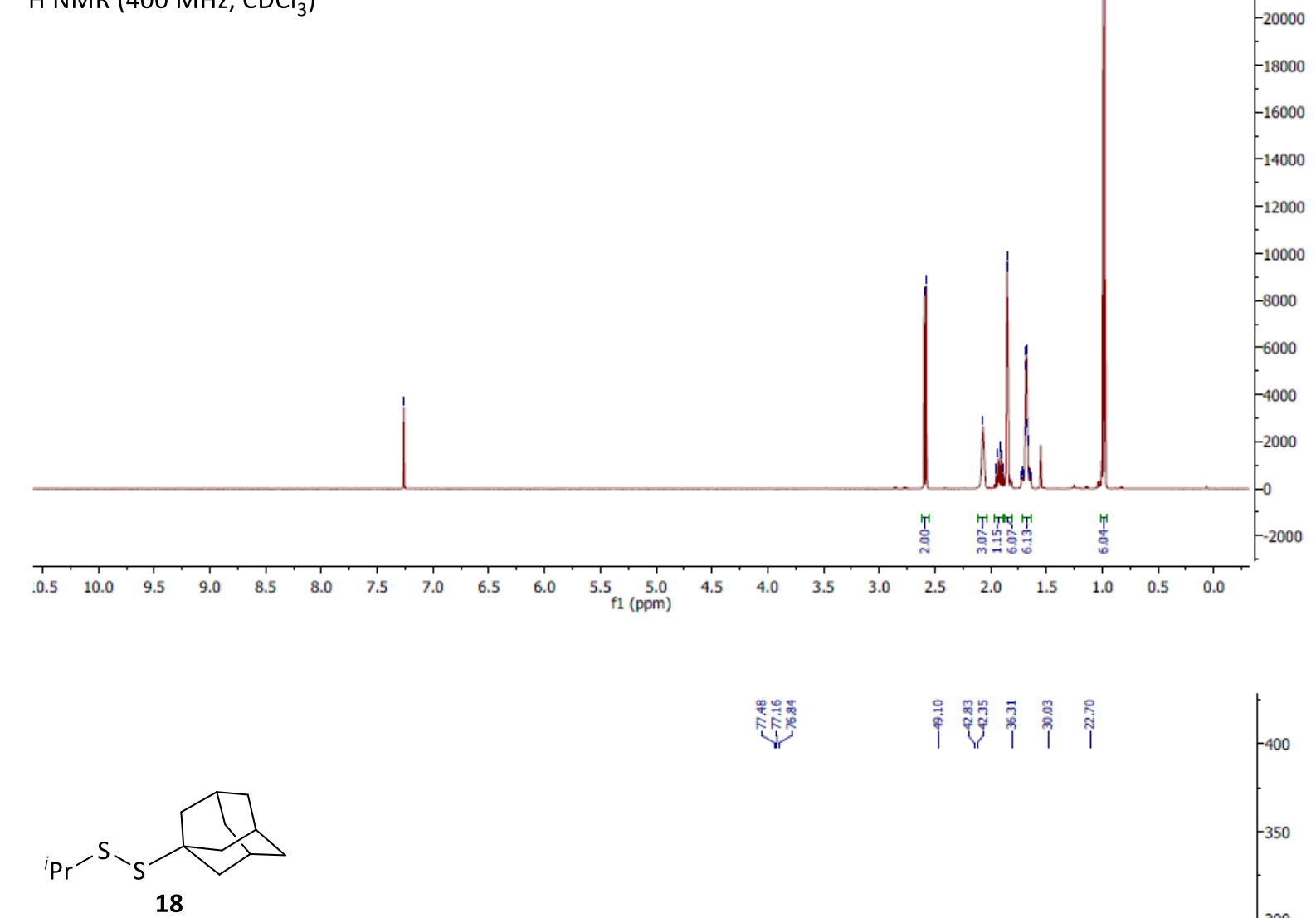

${ }^{13} \mathrm{CNMR}\left(101 \mathrm{MHz}, \mathrm{CDCl}_{3}\right)$
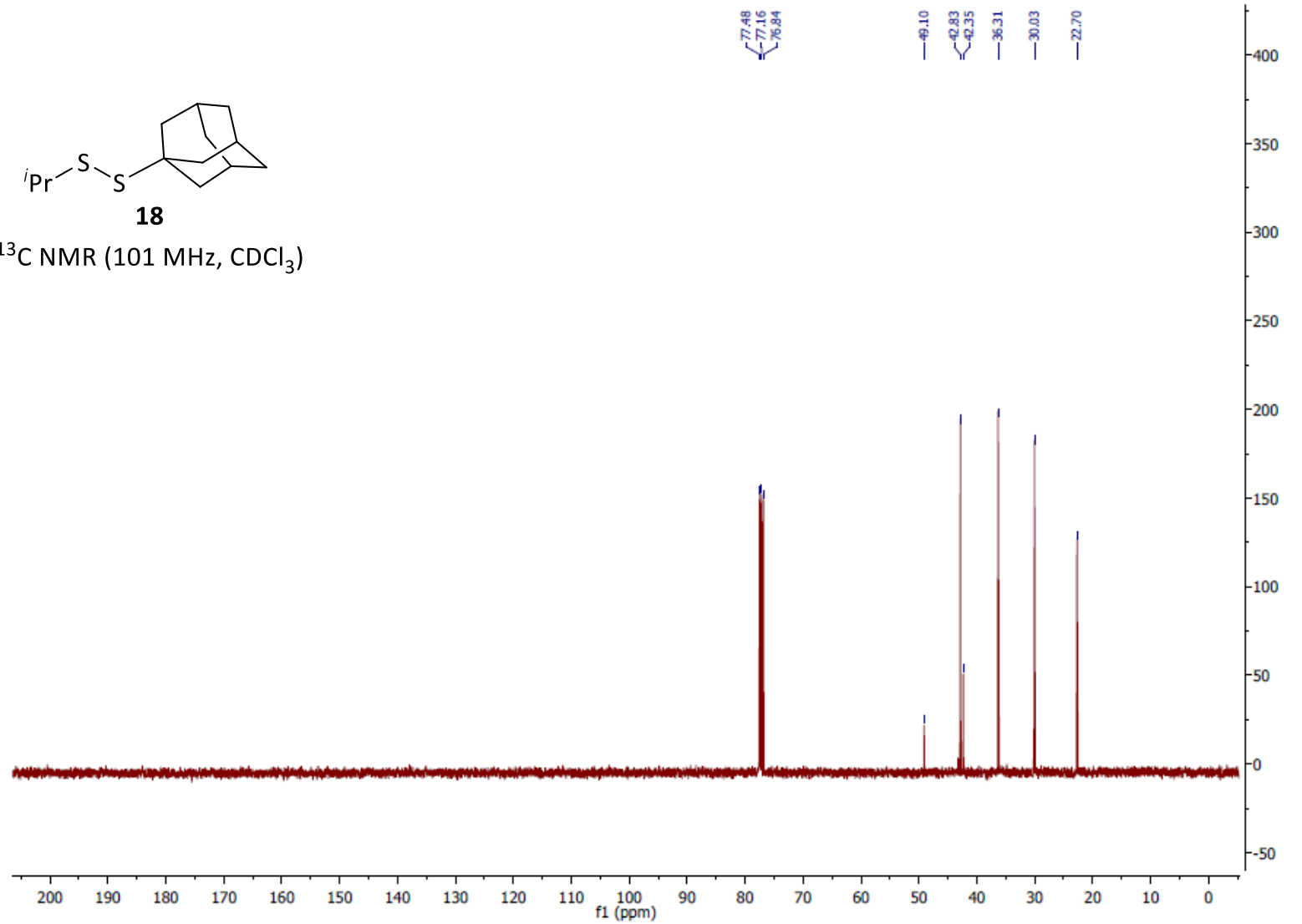


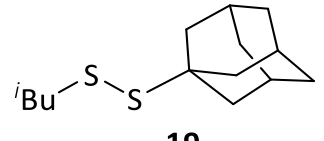

19

${ }^{1} \mathrm{H}$ NMR (400 MHz, $\mathrm{CDCl}_{3}$ )
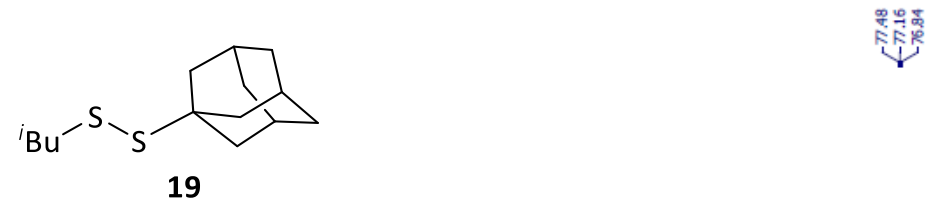

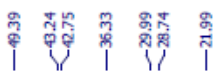

900

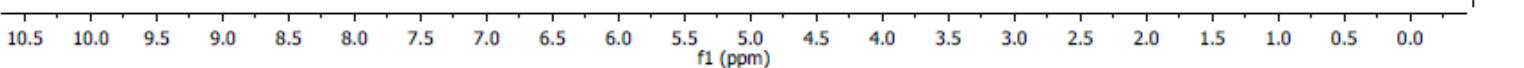

${ }^{13} \mathrm{C} \mathrm{NMR} \mathrm{(101} \mathrm{MHz,} \mathrm{CDCl}_{3}$ )

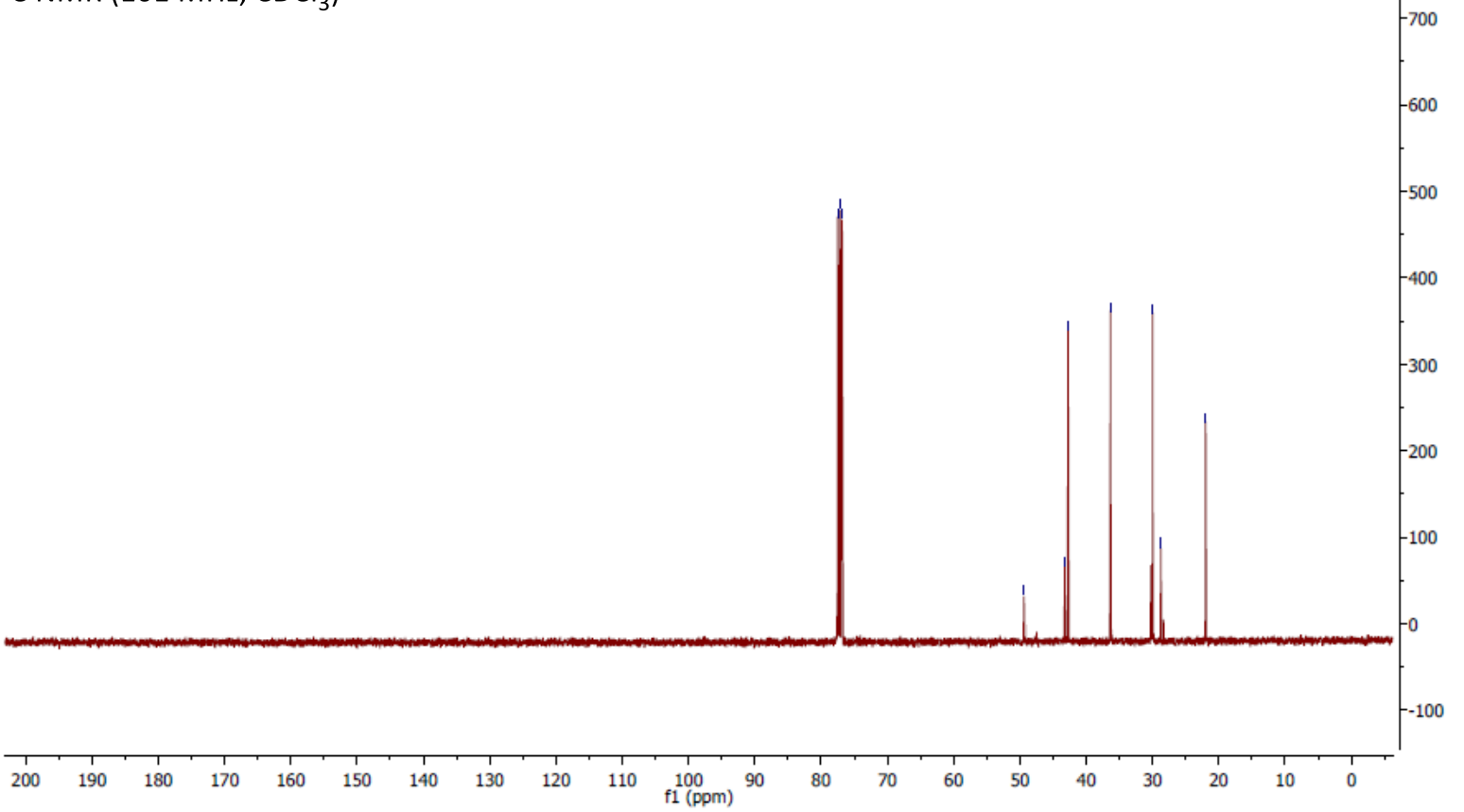



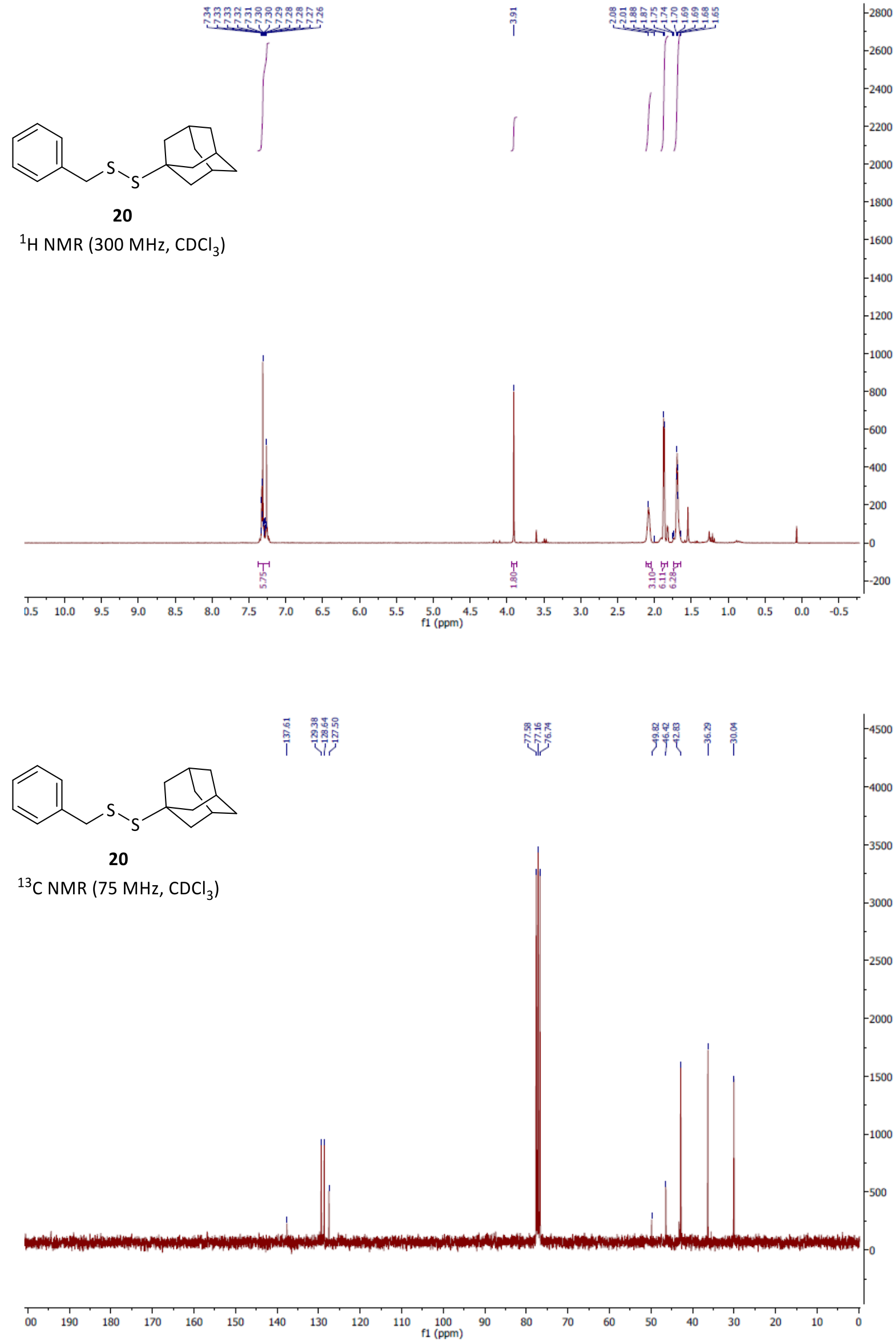

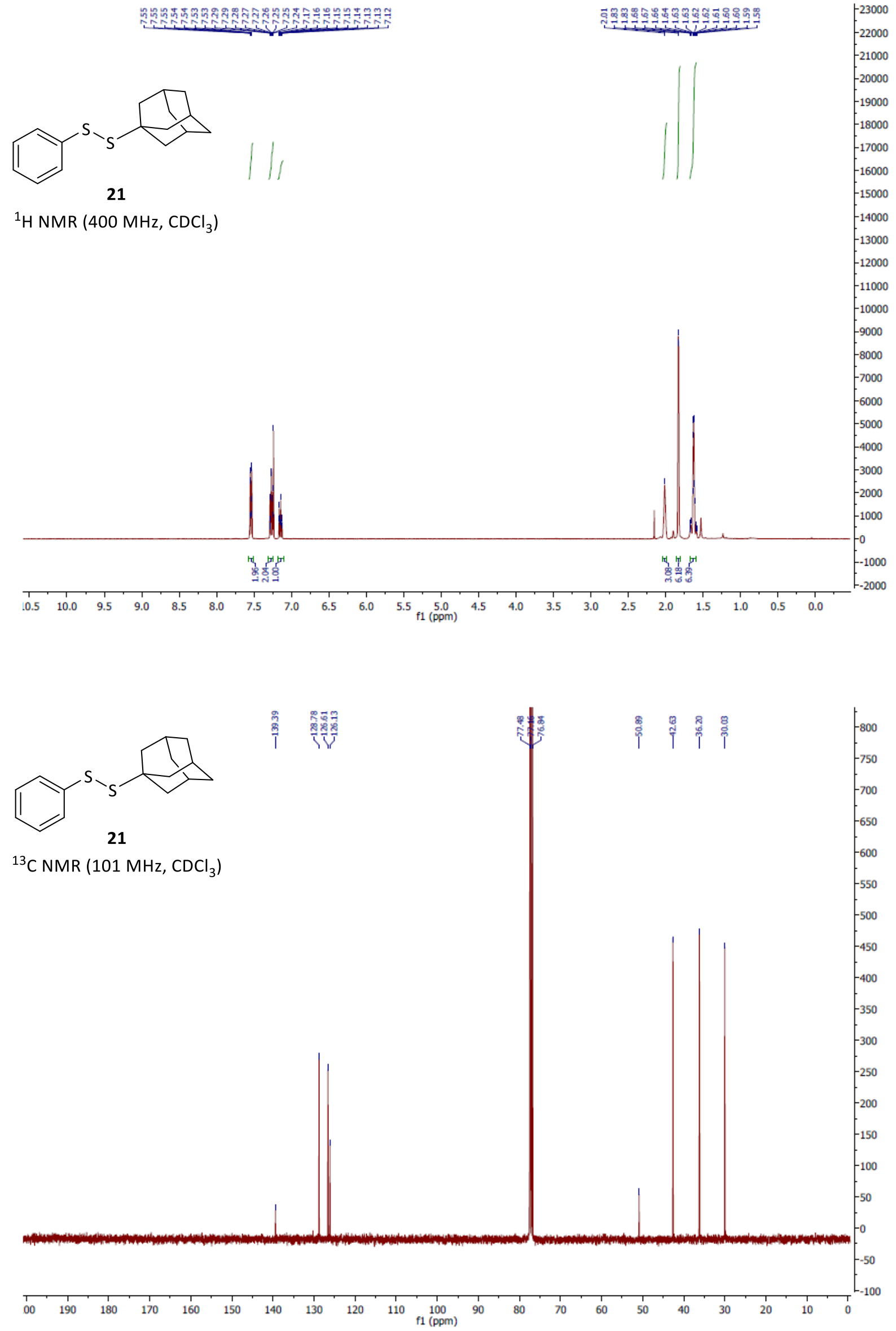

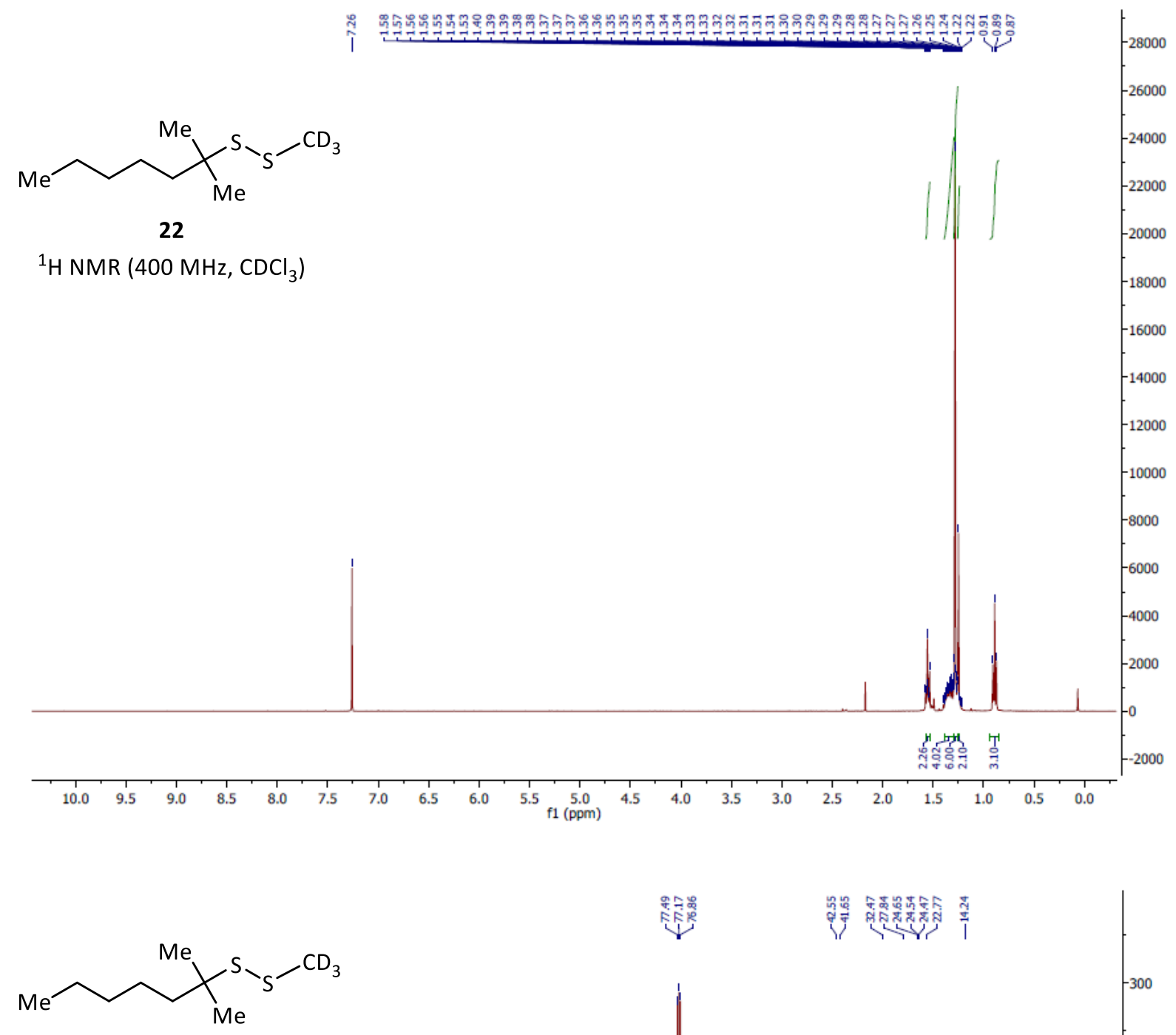

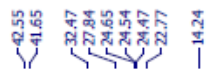

22

${ }^{13} \mathrm{C}$ NMR (101 MHz, $\mathrm{CDCl}_{3}$ )

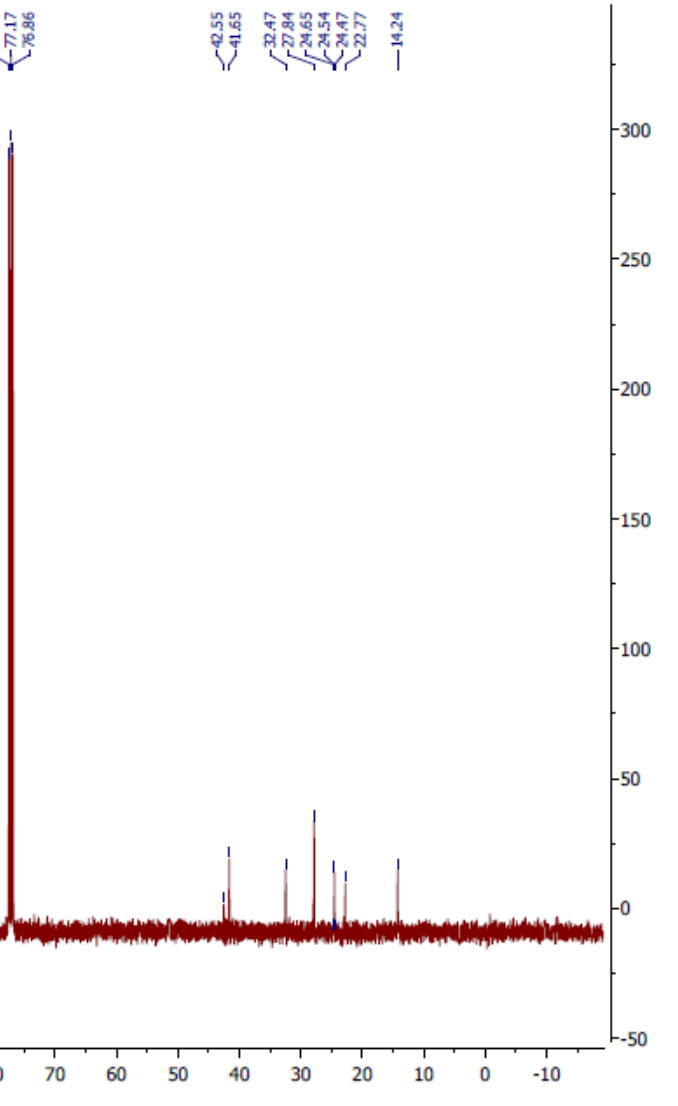




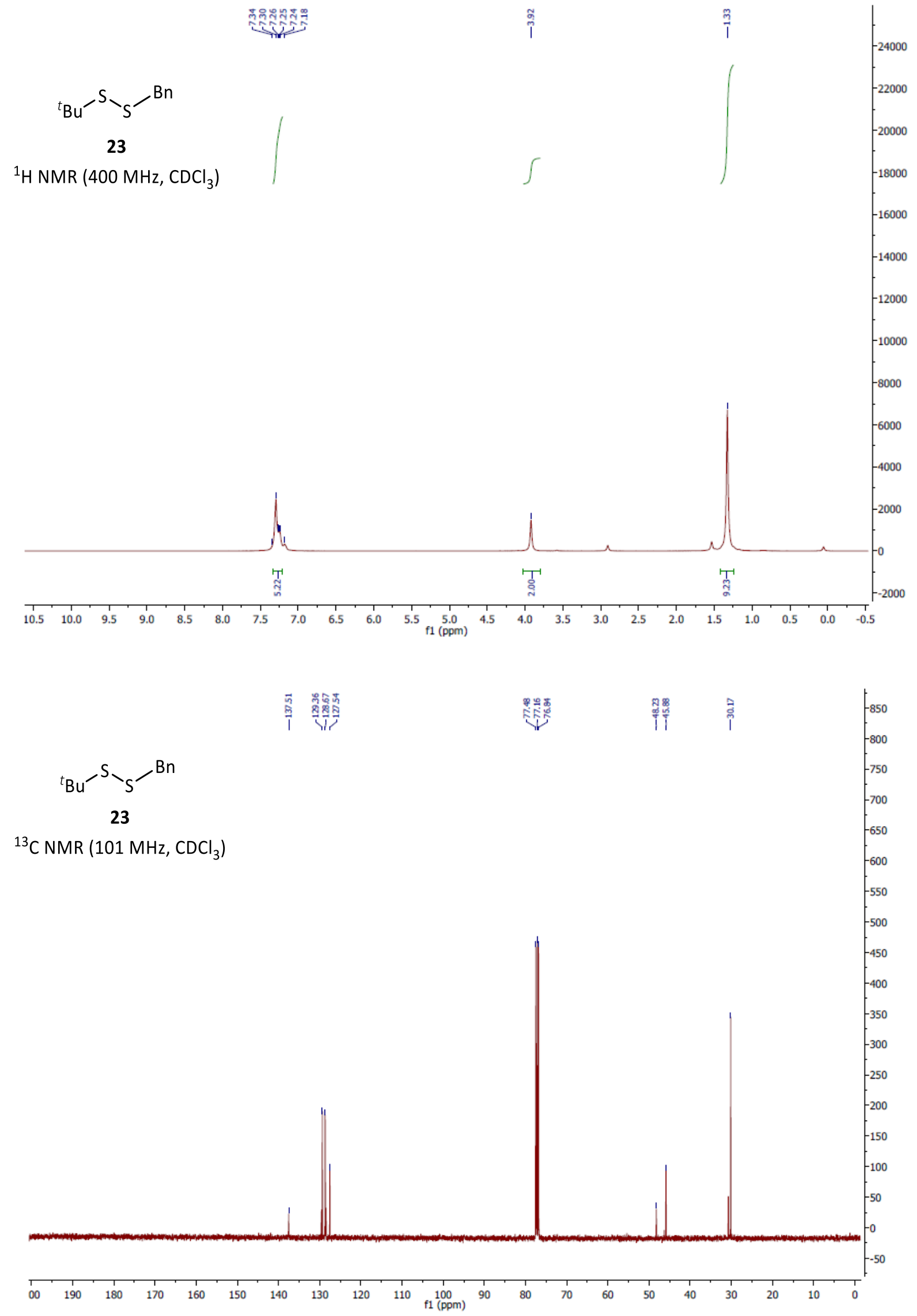



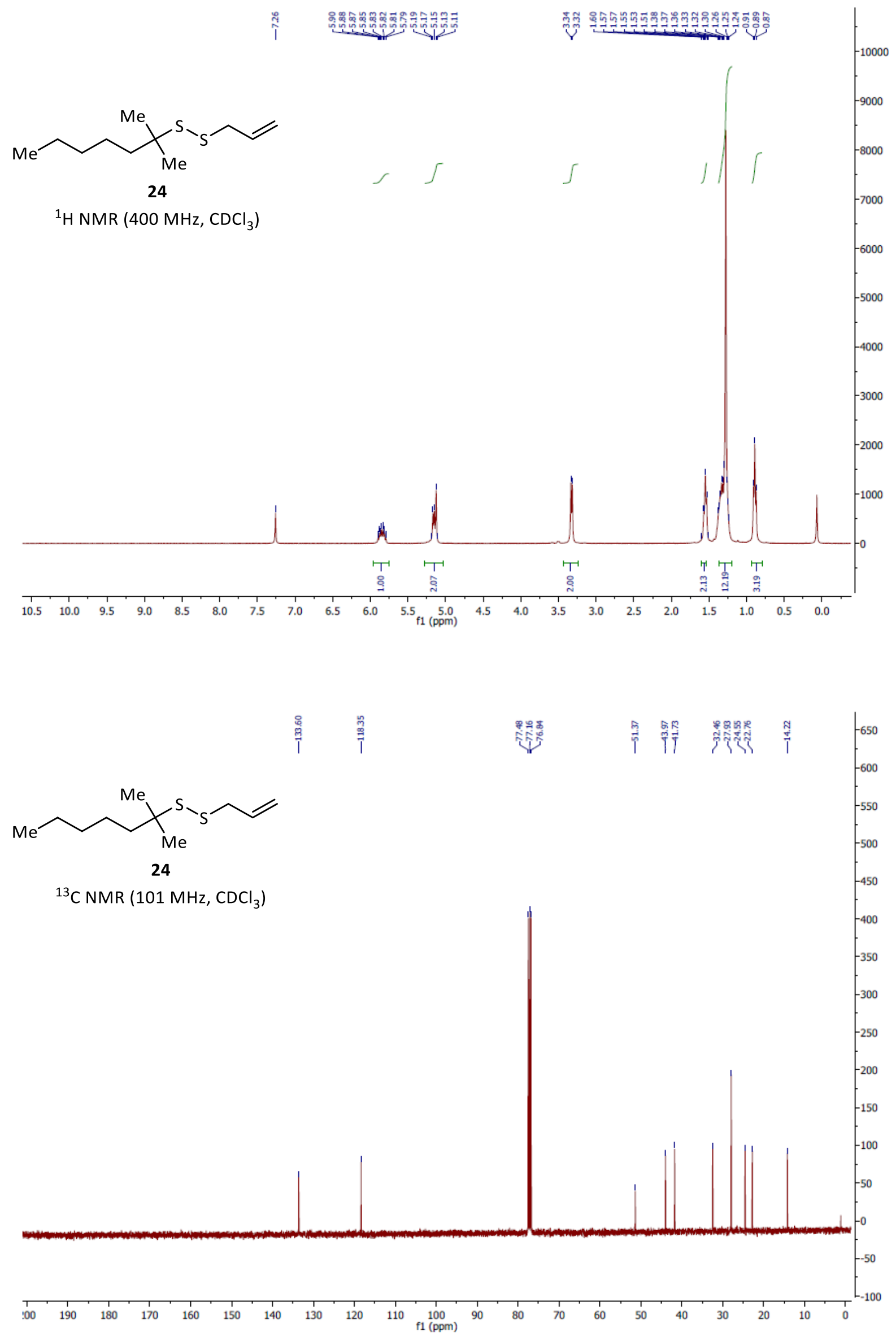

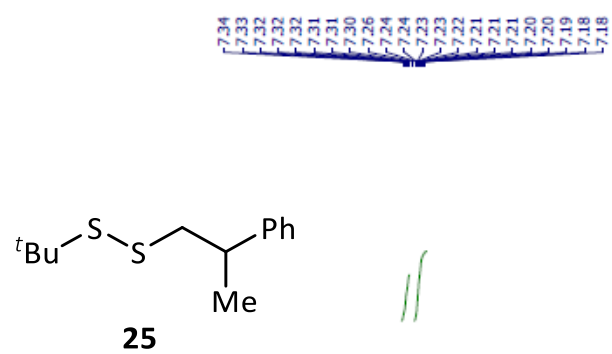

${ }^{1} \mathrm{H}$ NMR (400 MHz, $\mathrm{CDCl}_{3}$ )
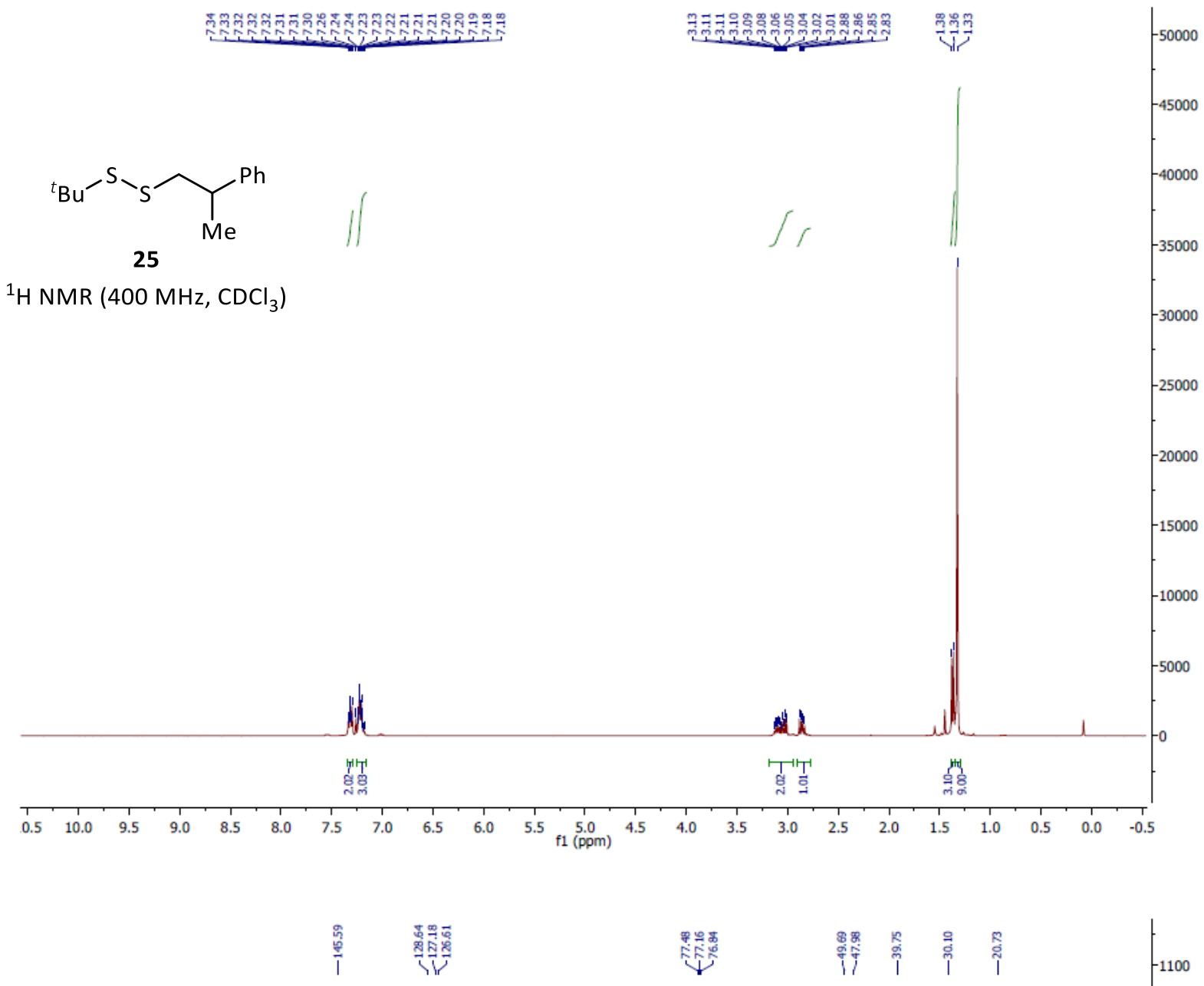

50000

5000

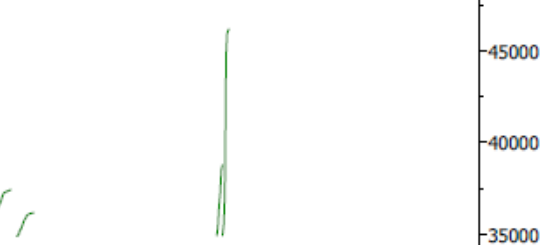

$-35000$

000

000

0000

5000

000

000 

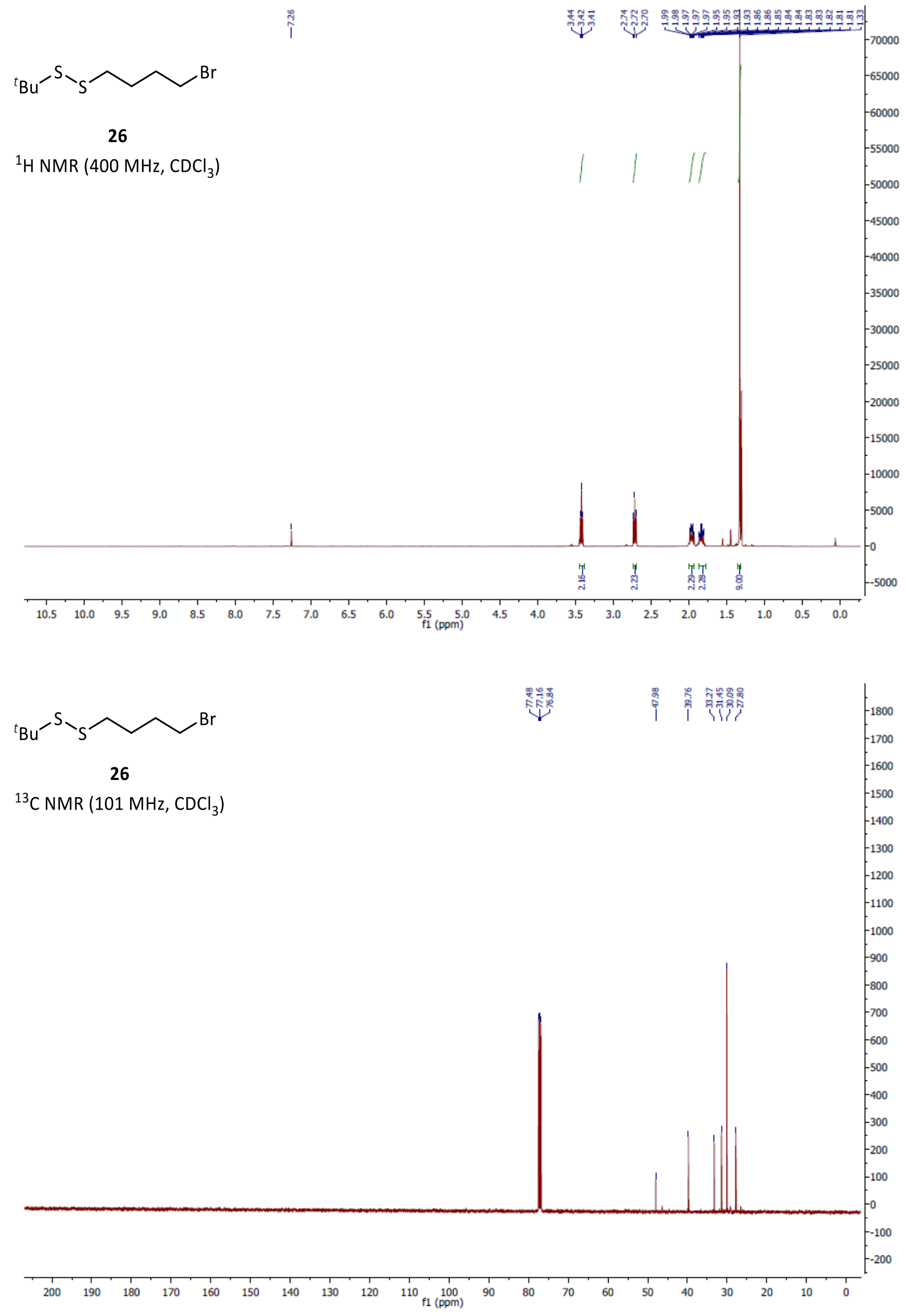

$\left.{ }^{13} \mathrm{C} \mathrm{NMR} \mathrm{(101} \mathrm{MHz,} \mathrm{CDCl}\right)$ 
<smiles>CC(C)(C)SSCCC(=O)c1ccccc1</smiles>

${ }^{1} \mathrm{H}$ NMR $\left(300 \mathrm{MHz}, \mathrm{CDCl}_{3}\right) / / /$
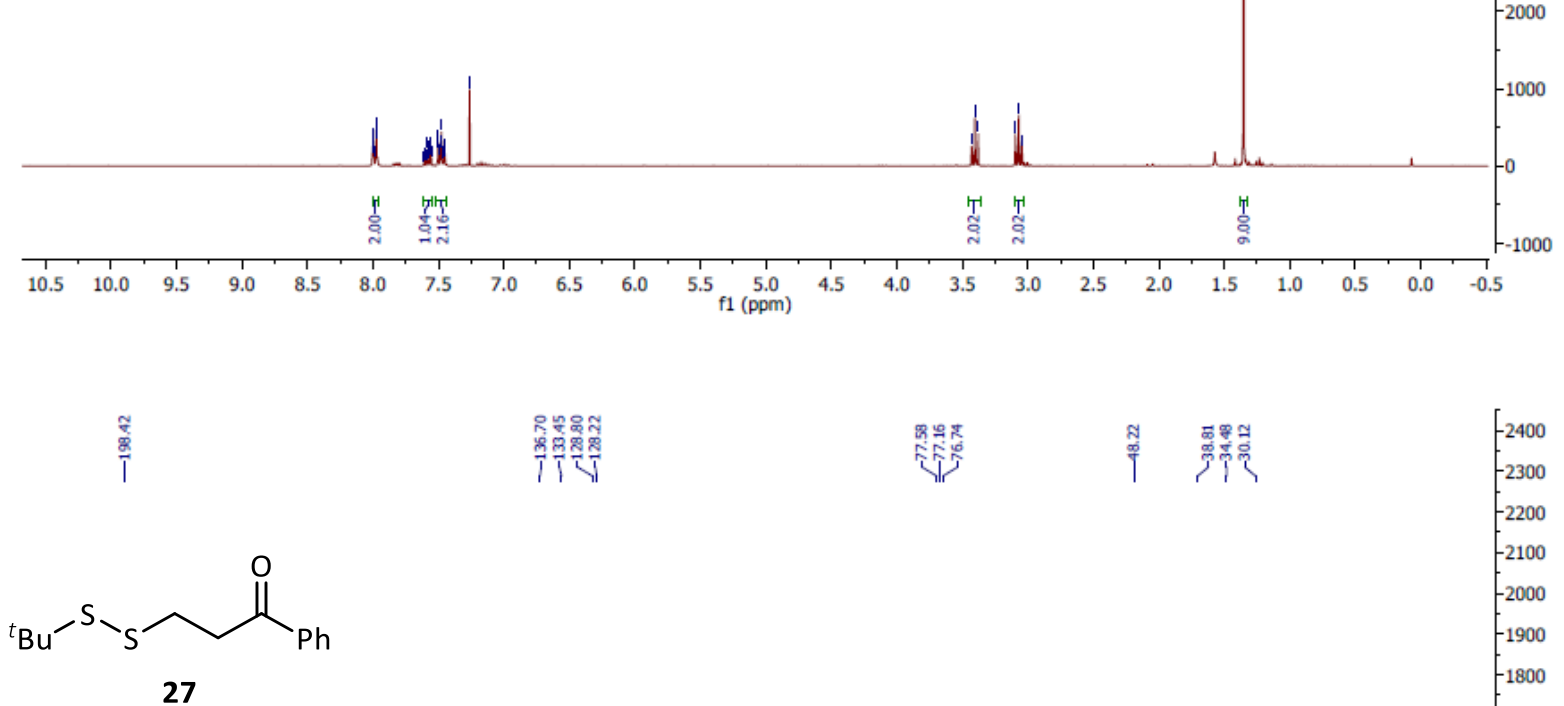

${ }^{13} \mathrm{C} \mathrm{NMR}\left(75 \mathrm{MHz}, \mathrm{CDCl}_{3}\right)$ 

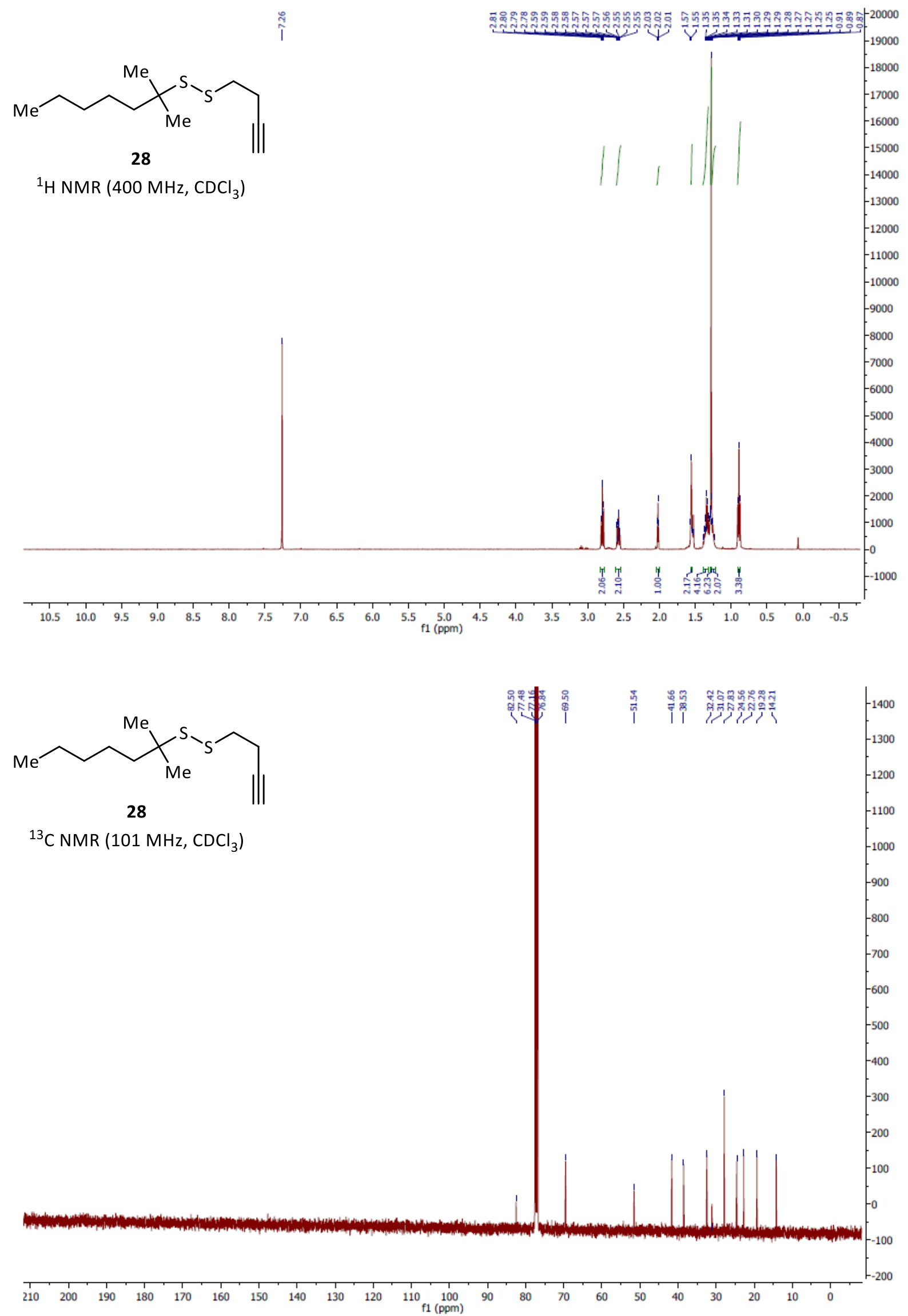

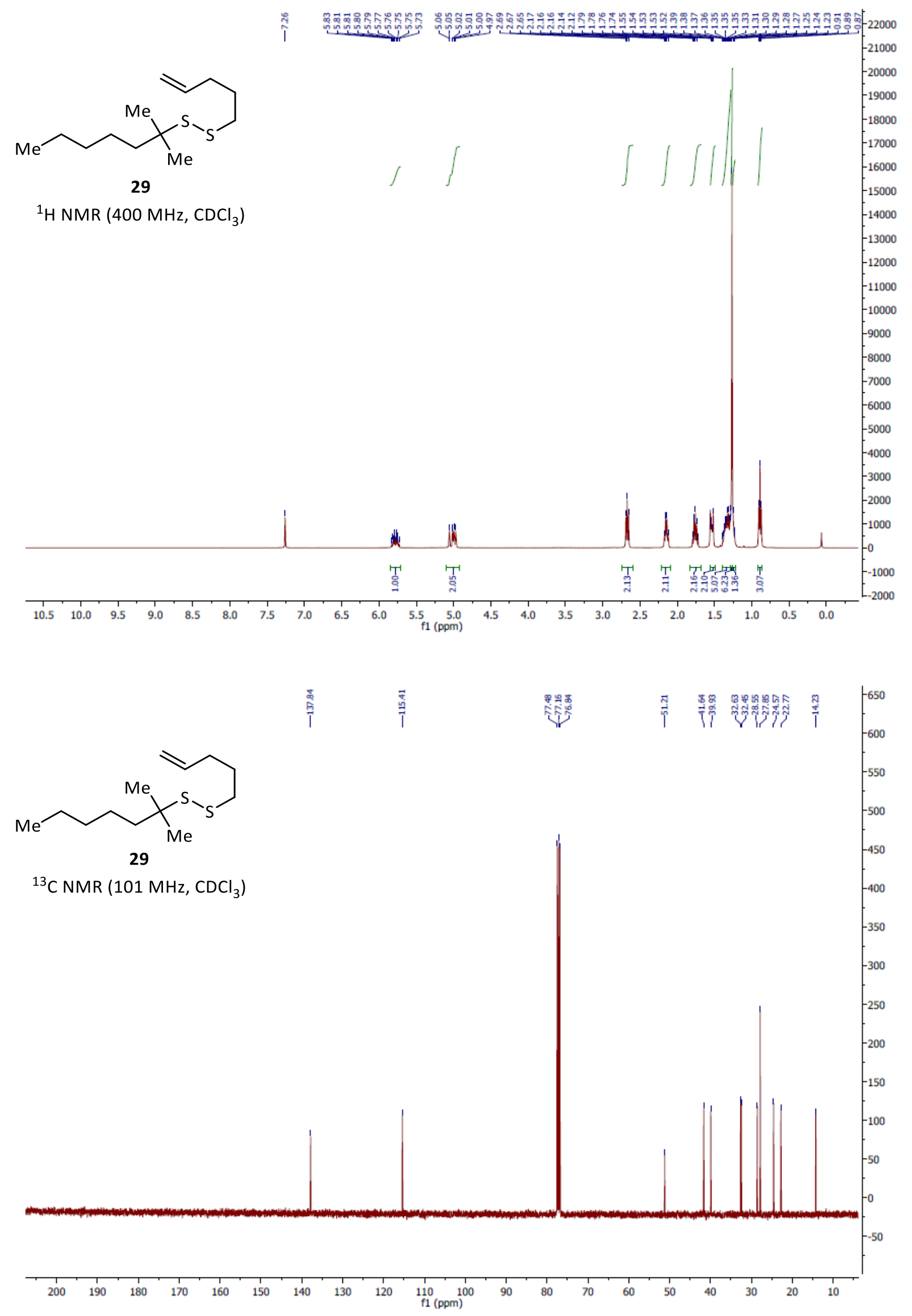

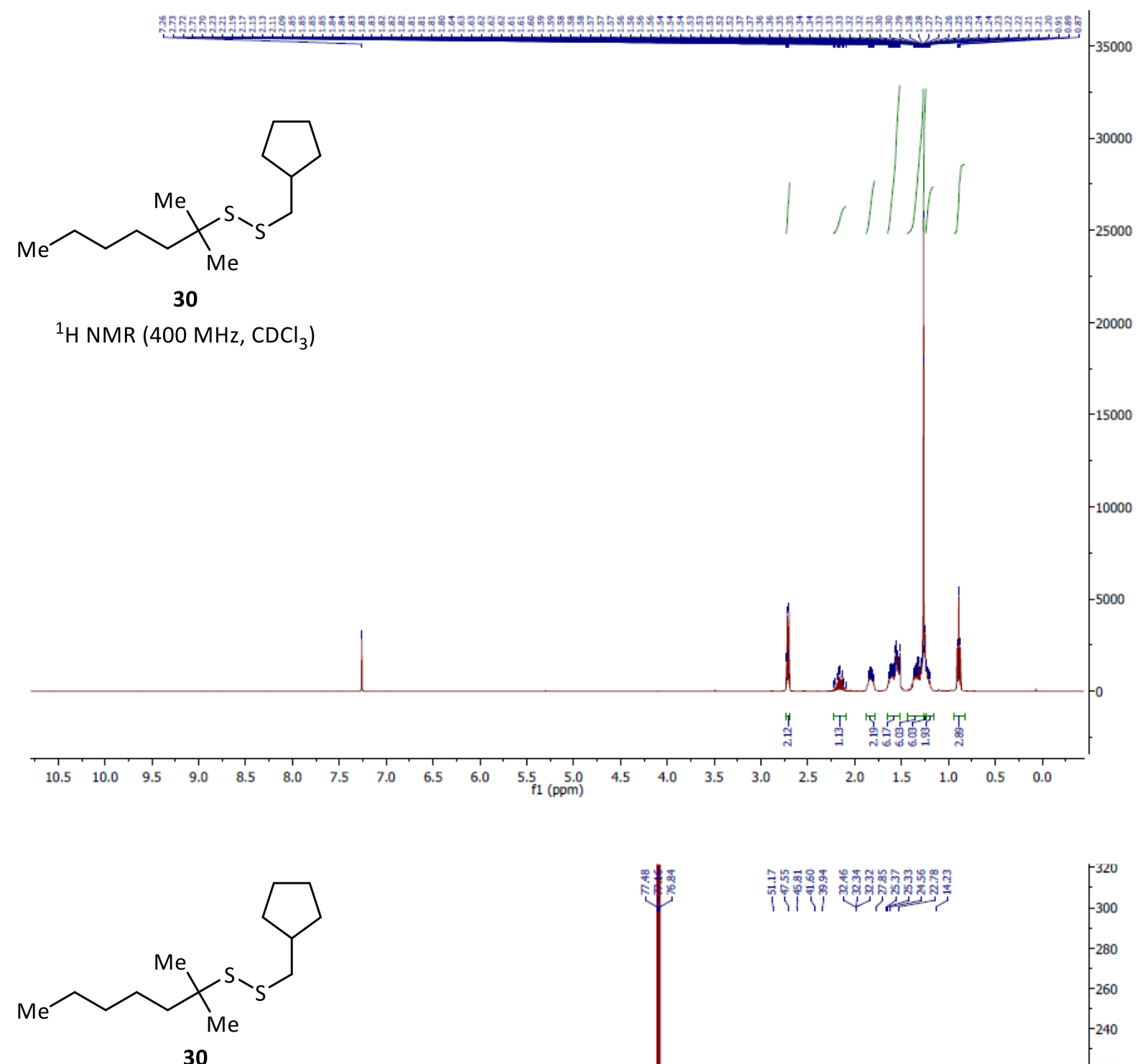

${ }^{13} \mathrm{C}$ NMR (101 MHz, $\mathrm{CDCl}_{3}$ )

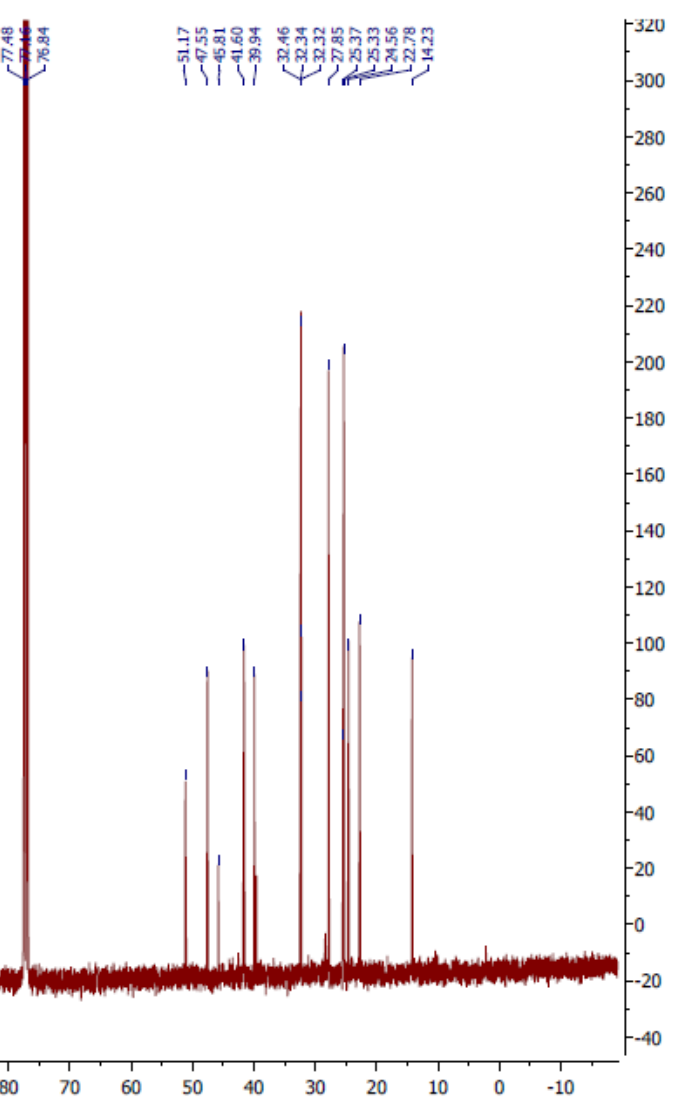



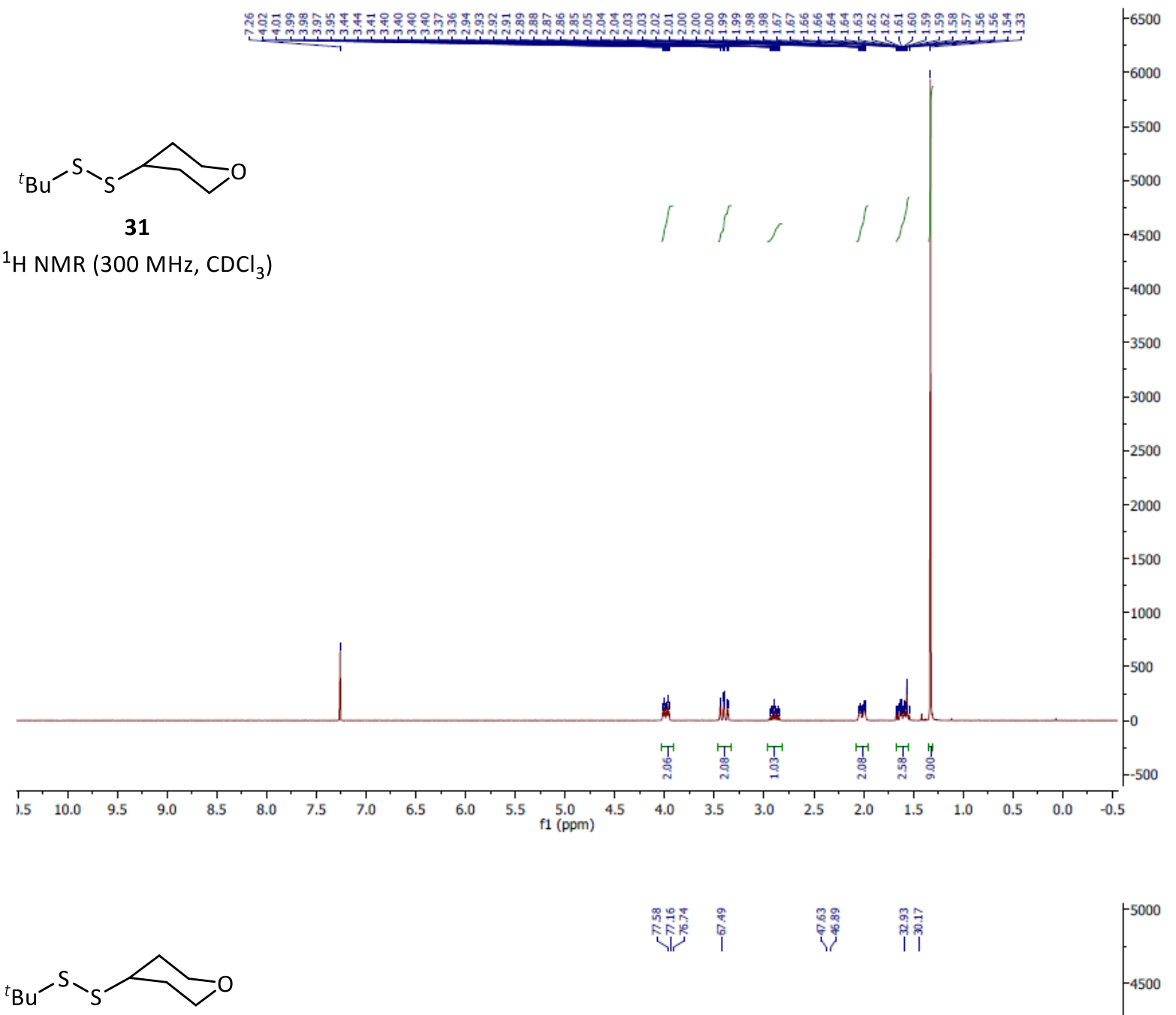

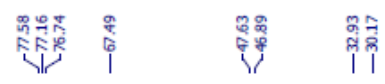

31

${ }^{13} \mathrm{C} \mathrm{NMR}\left(75 \mathrm{MHz}, \mathrm{CDCl}_{3}\right)$

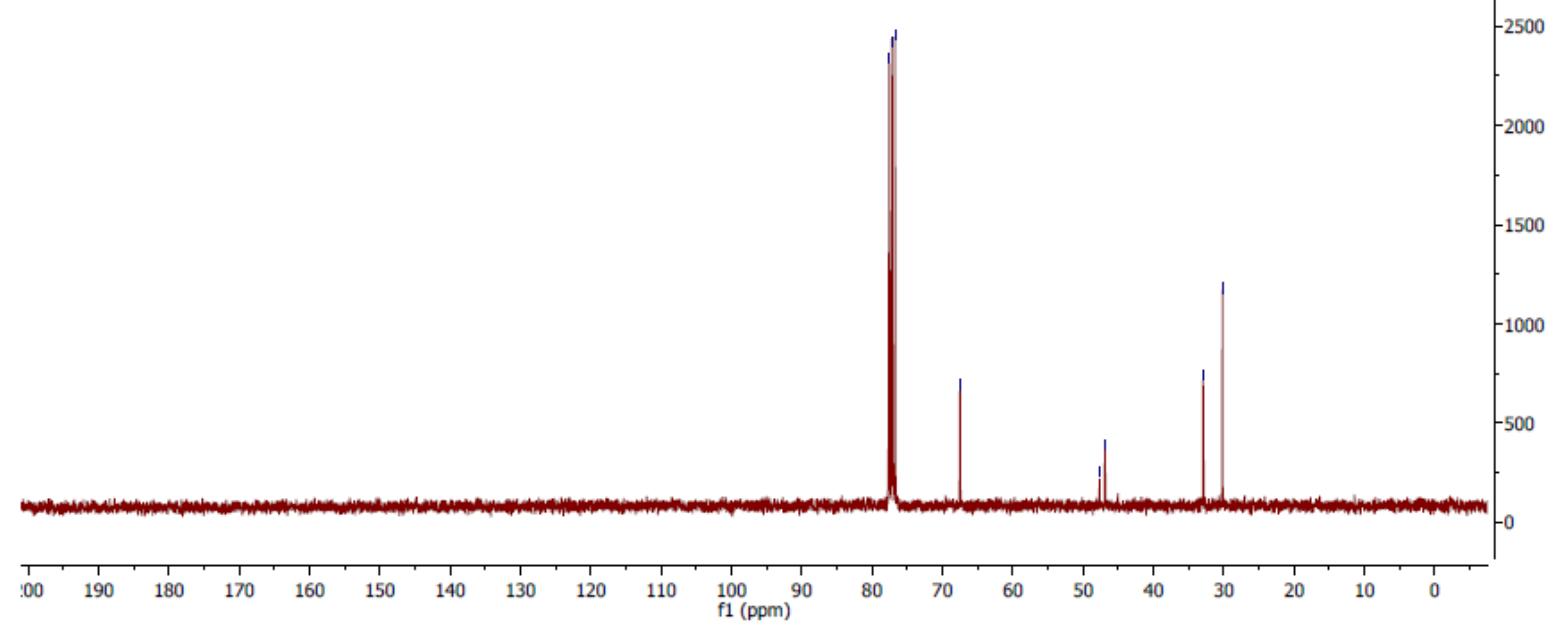



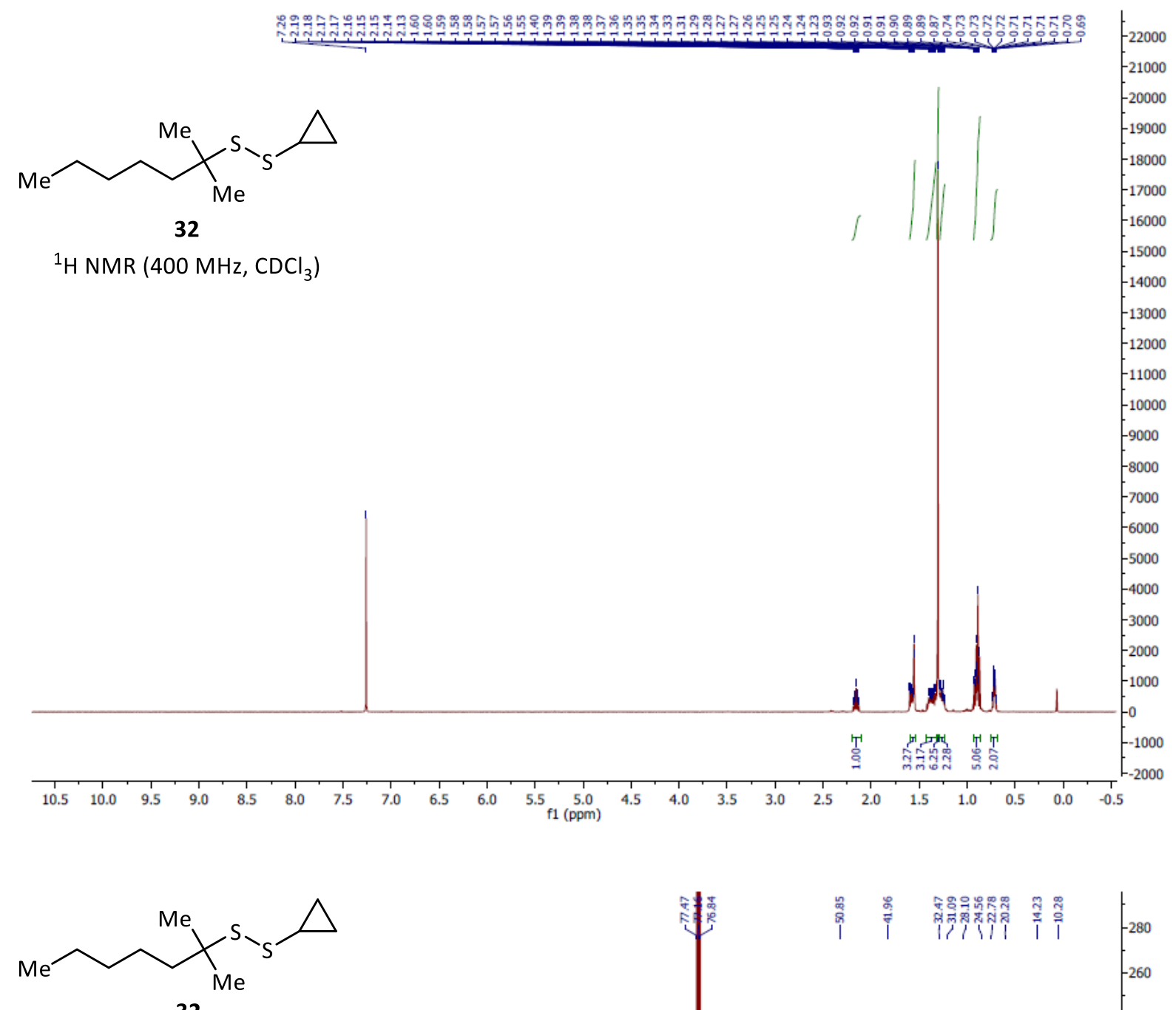

32

${ }^{13} \mathrm{C}$ NMR (101 MHz, $\left.\mathrm{CDCl}_{3}\right)$

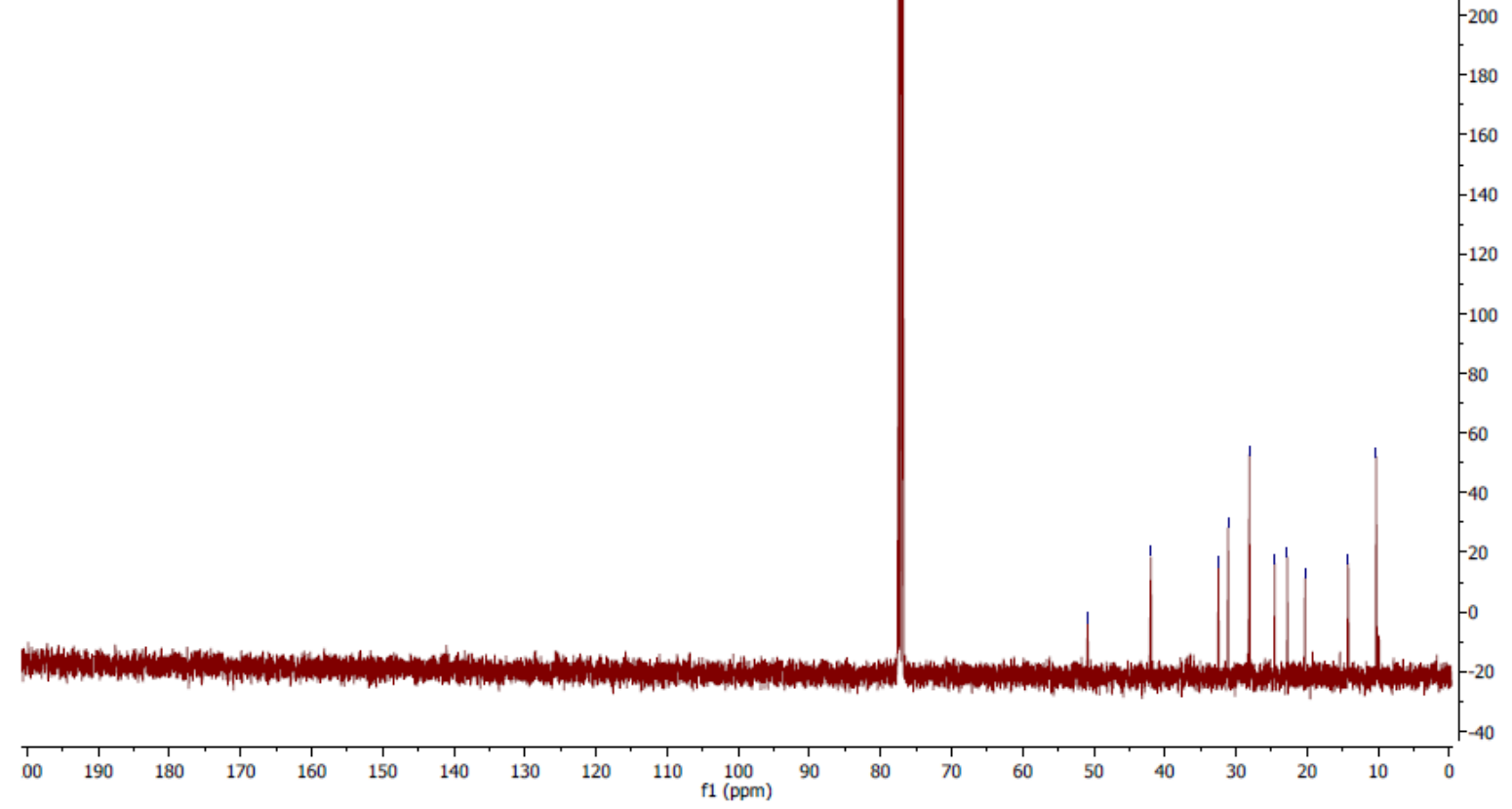



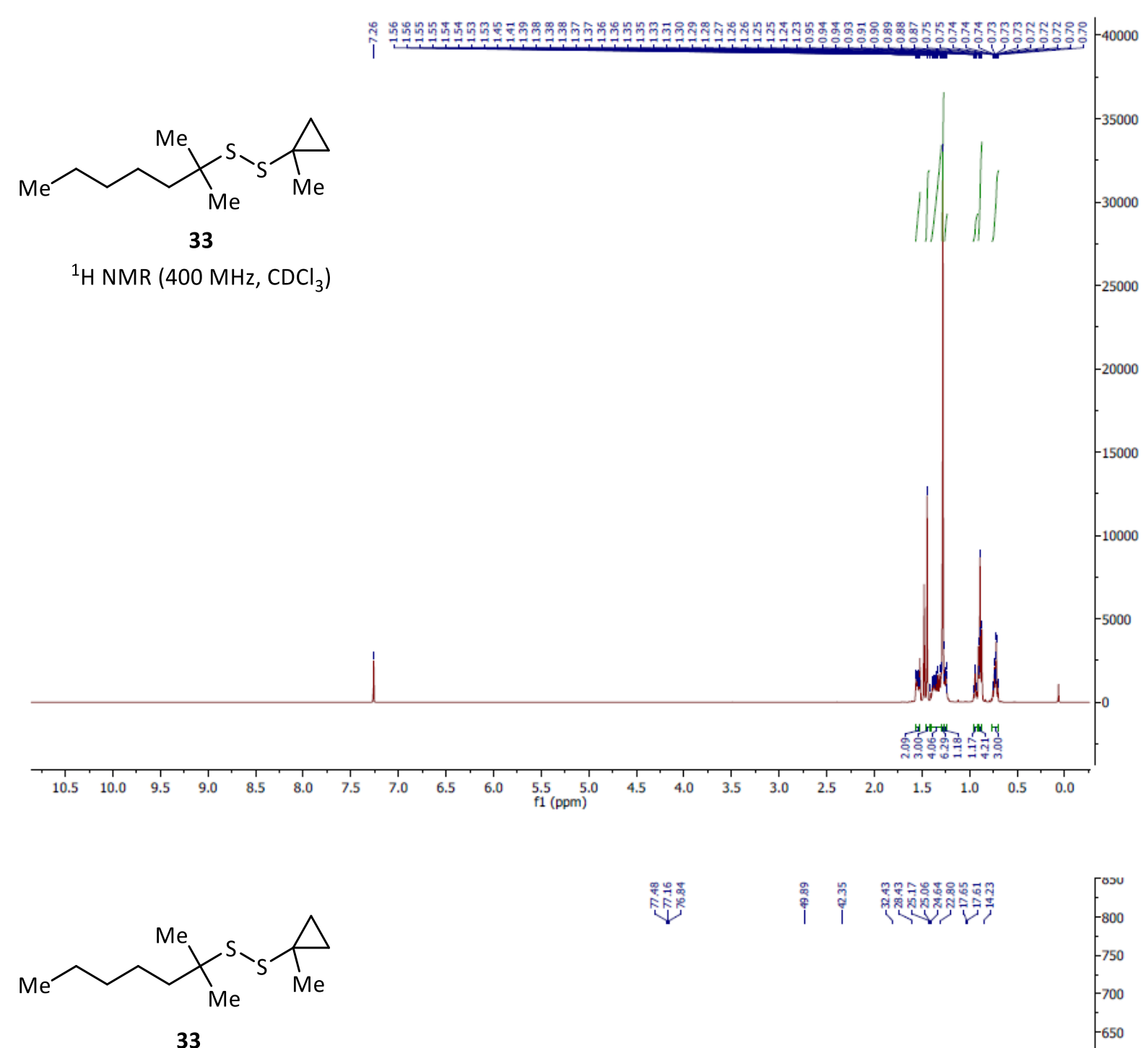

33

${ }^{13} \mathrm{C}$ NMR (101 MHz, CDCl 3 )

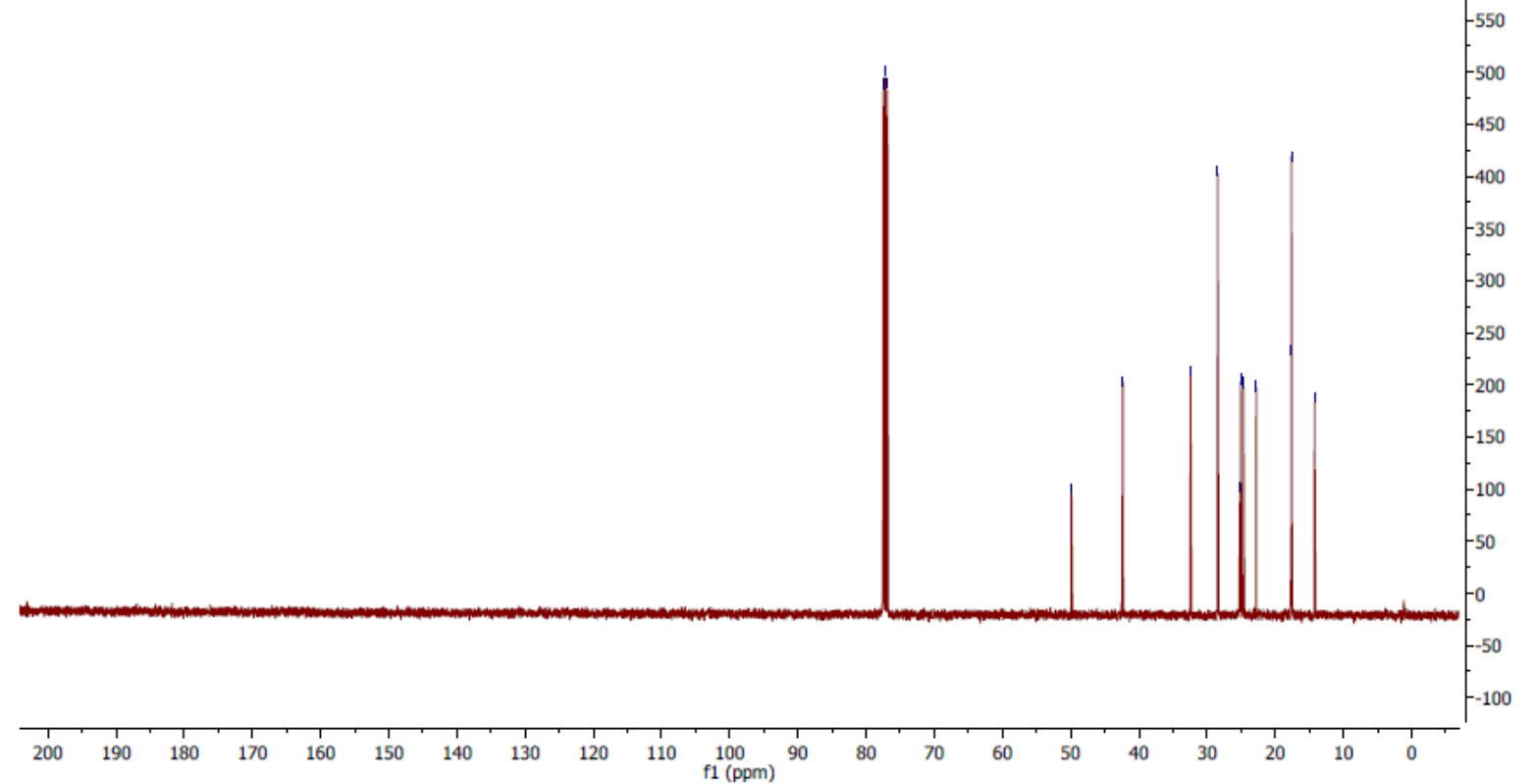




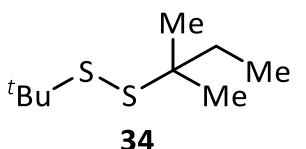

$\left.{ }^{1} \mathrm{H} \mathrm{NMR} \mathrm{(400} \mathrm{MHz,} \mathrm{CDCl}_{3}\right)$
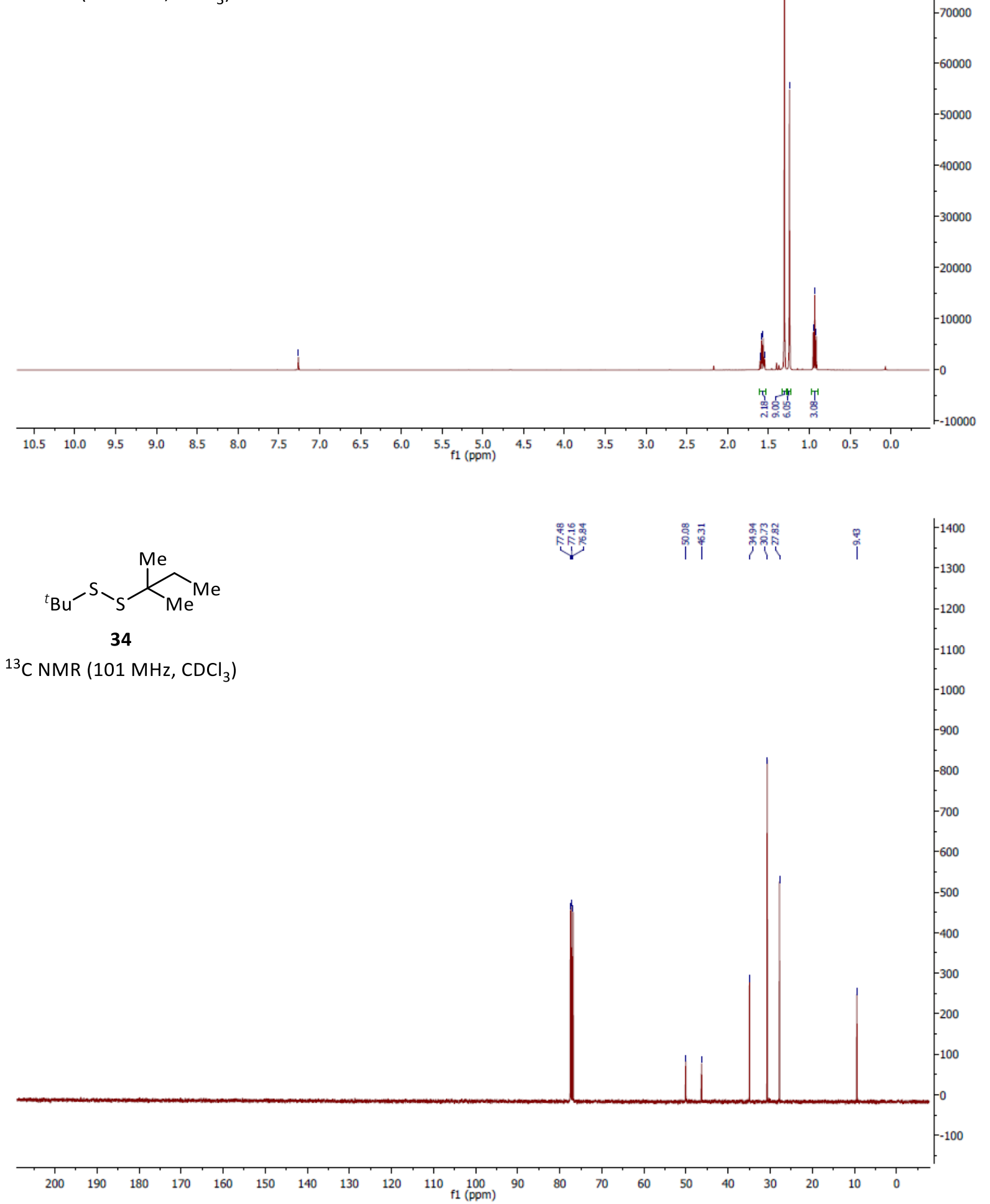

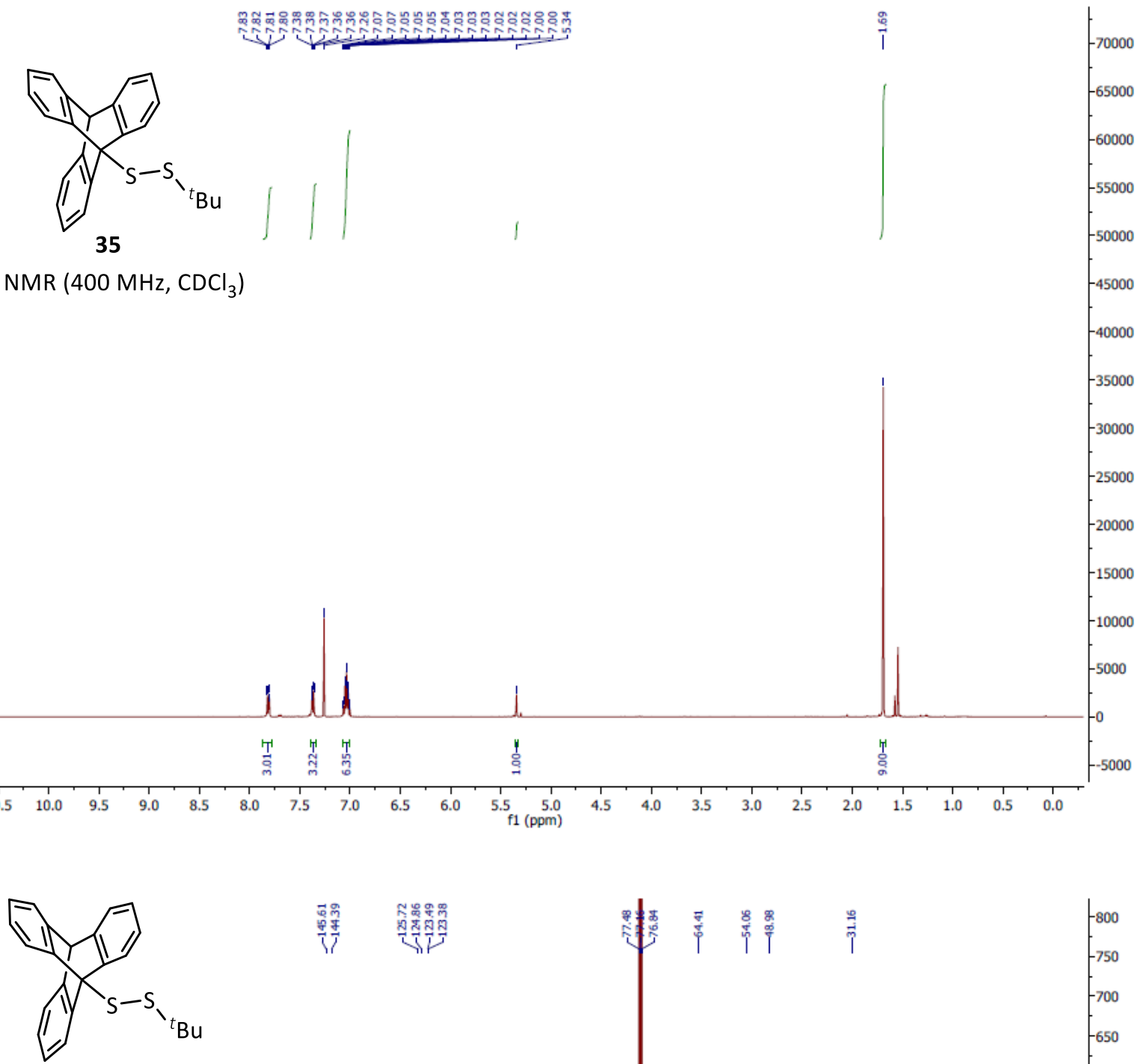

35

${ }^{13} \mathrm{C}$ NMR $\left(101 \mathrm{MHz}, \mathrm{CDCl}_{3}\right)$
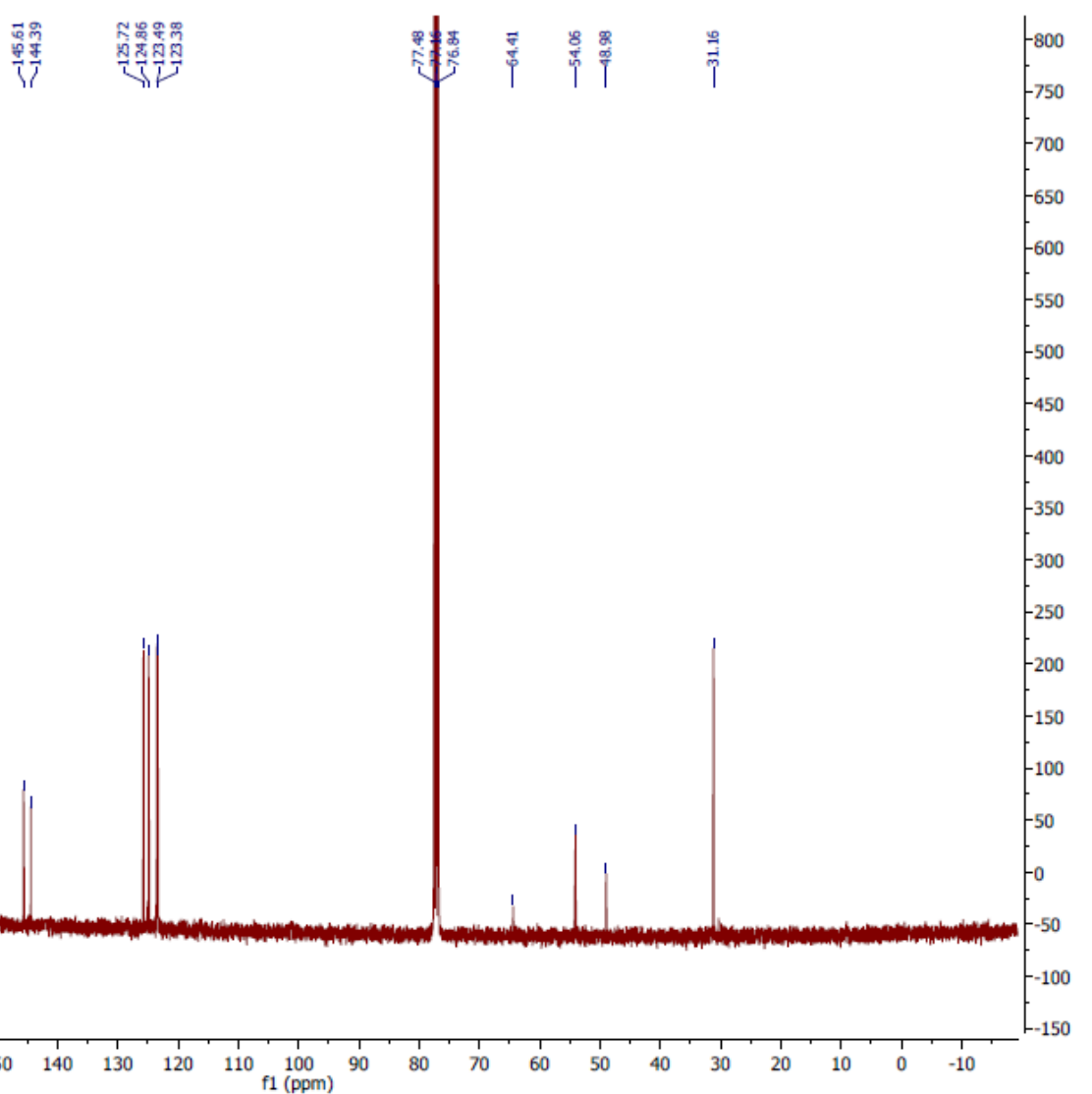

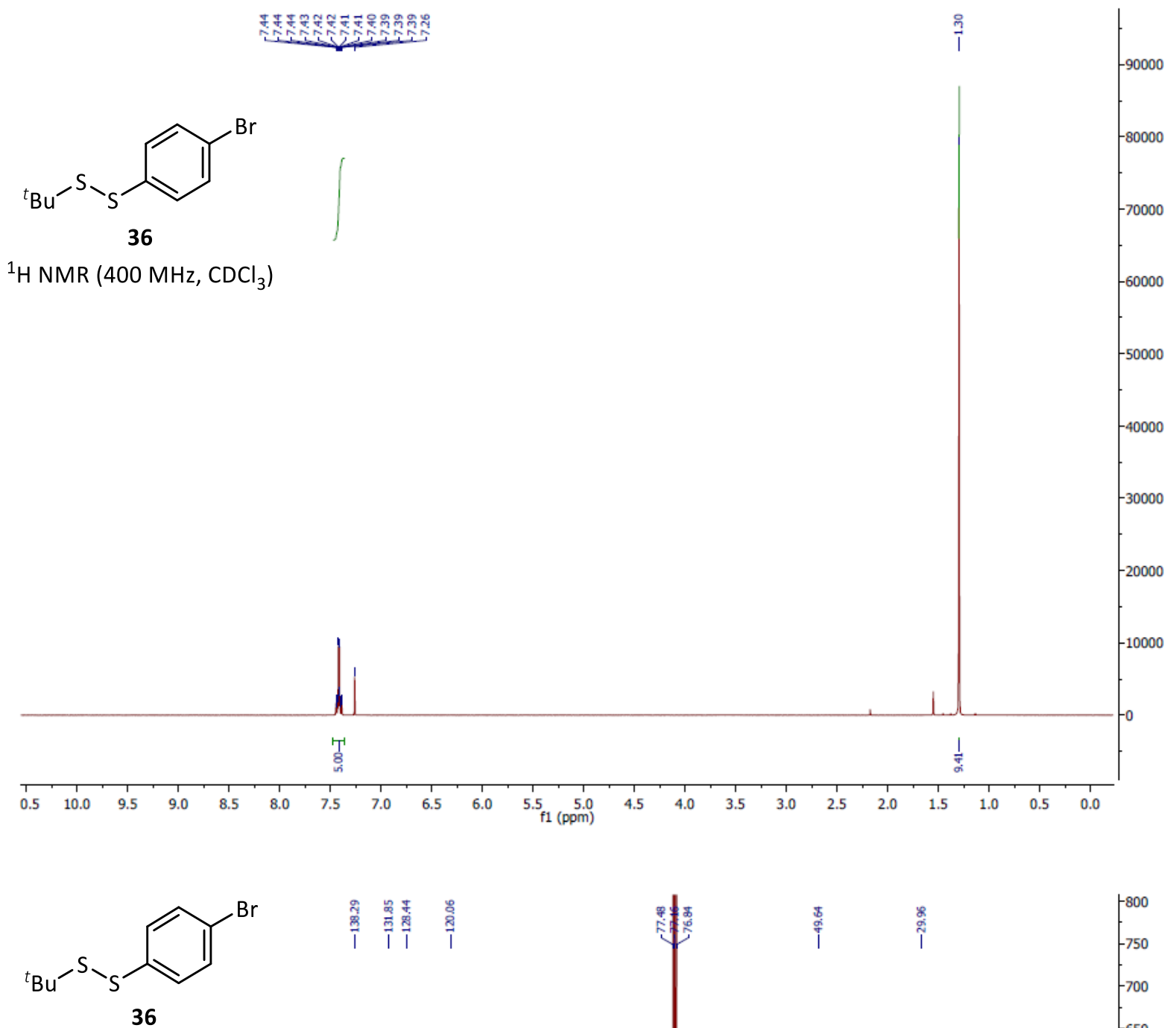

${ }^{13} \mathrm{C}$ NMR (101 MHz, CDCl $)$

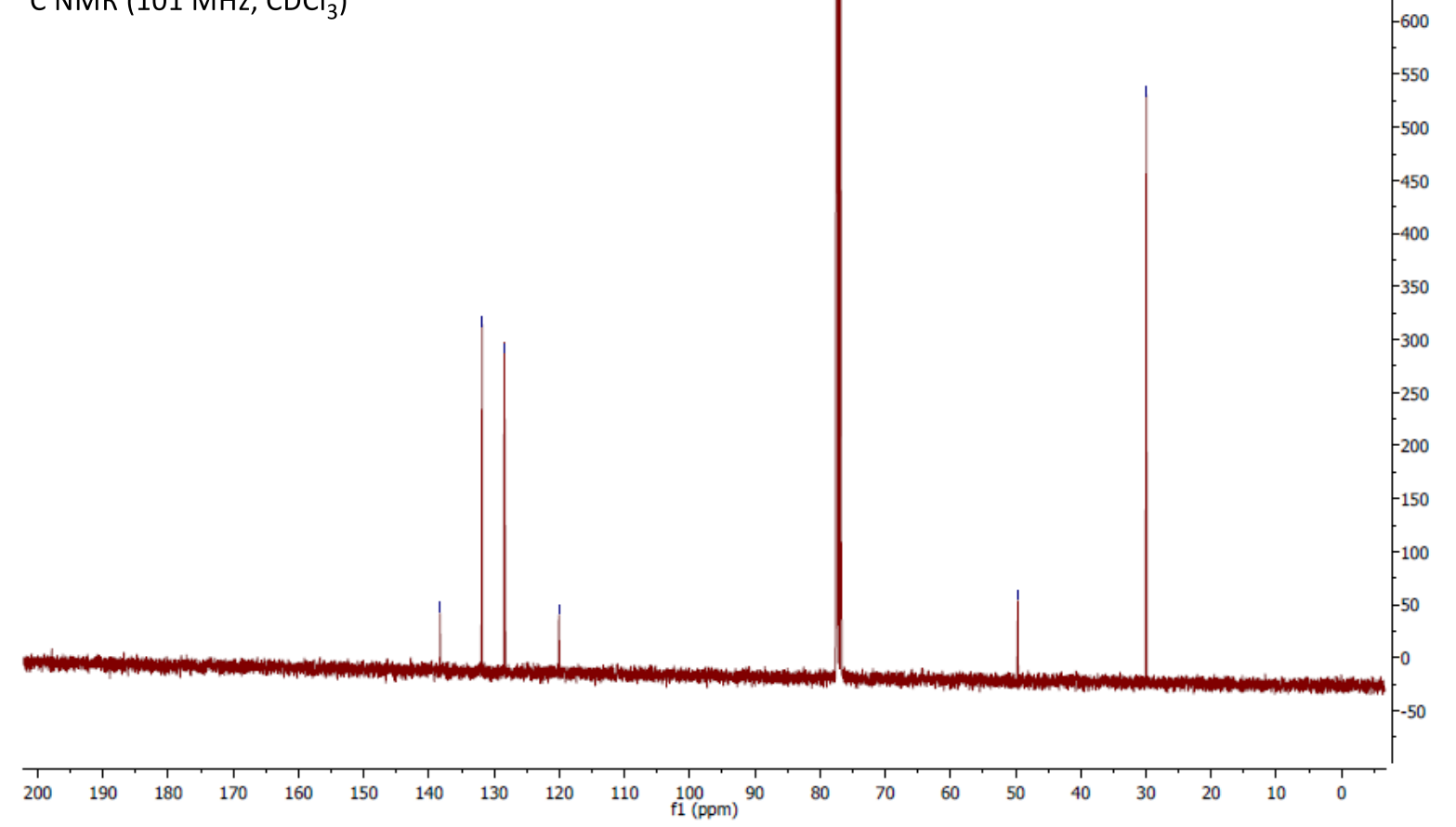



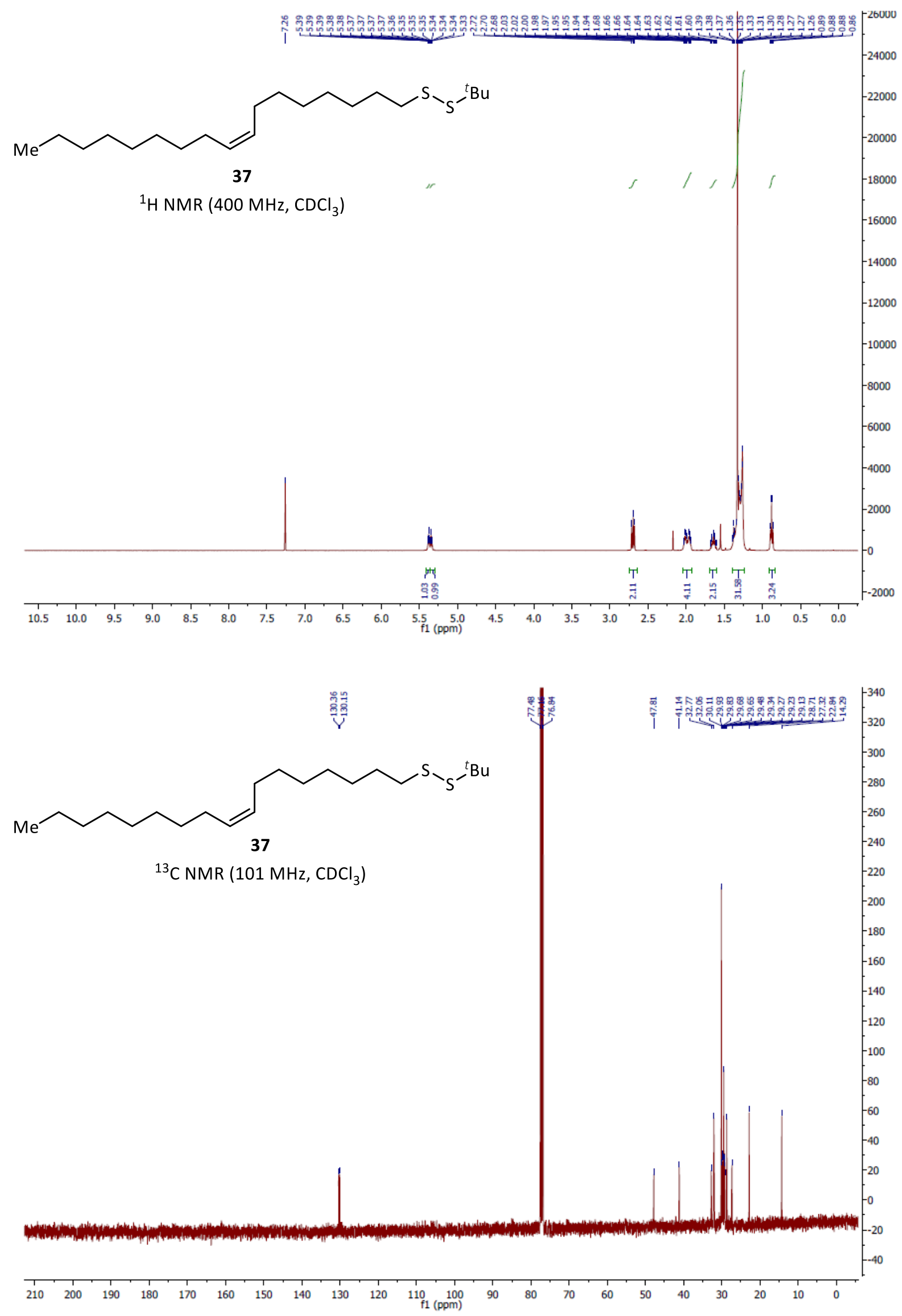

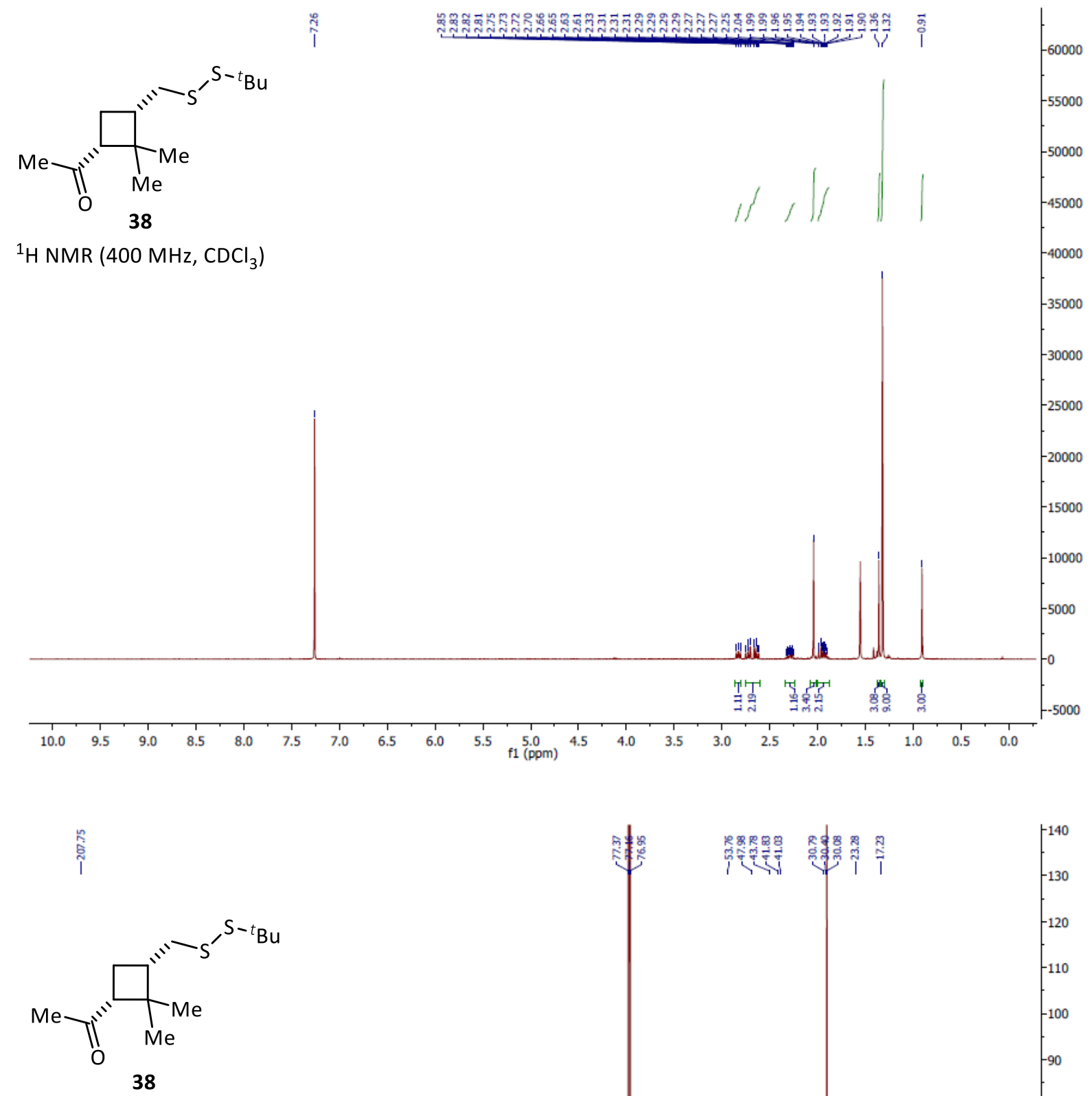

${ }^{13} \mathrm{C}$ NMR (151 MHz, $\mathrm{CDCl}_{3}$ )

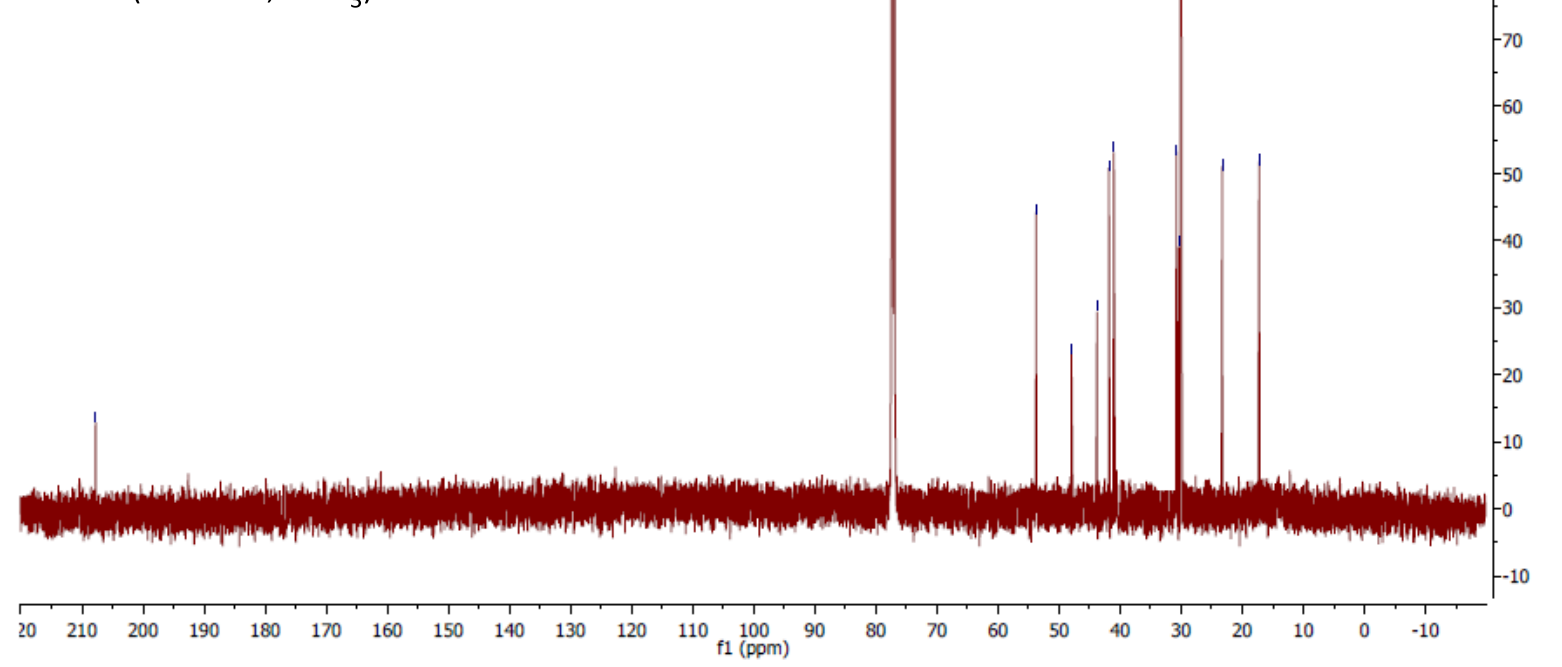



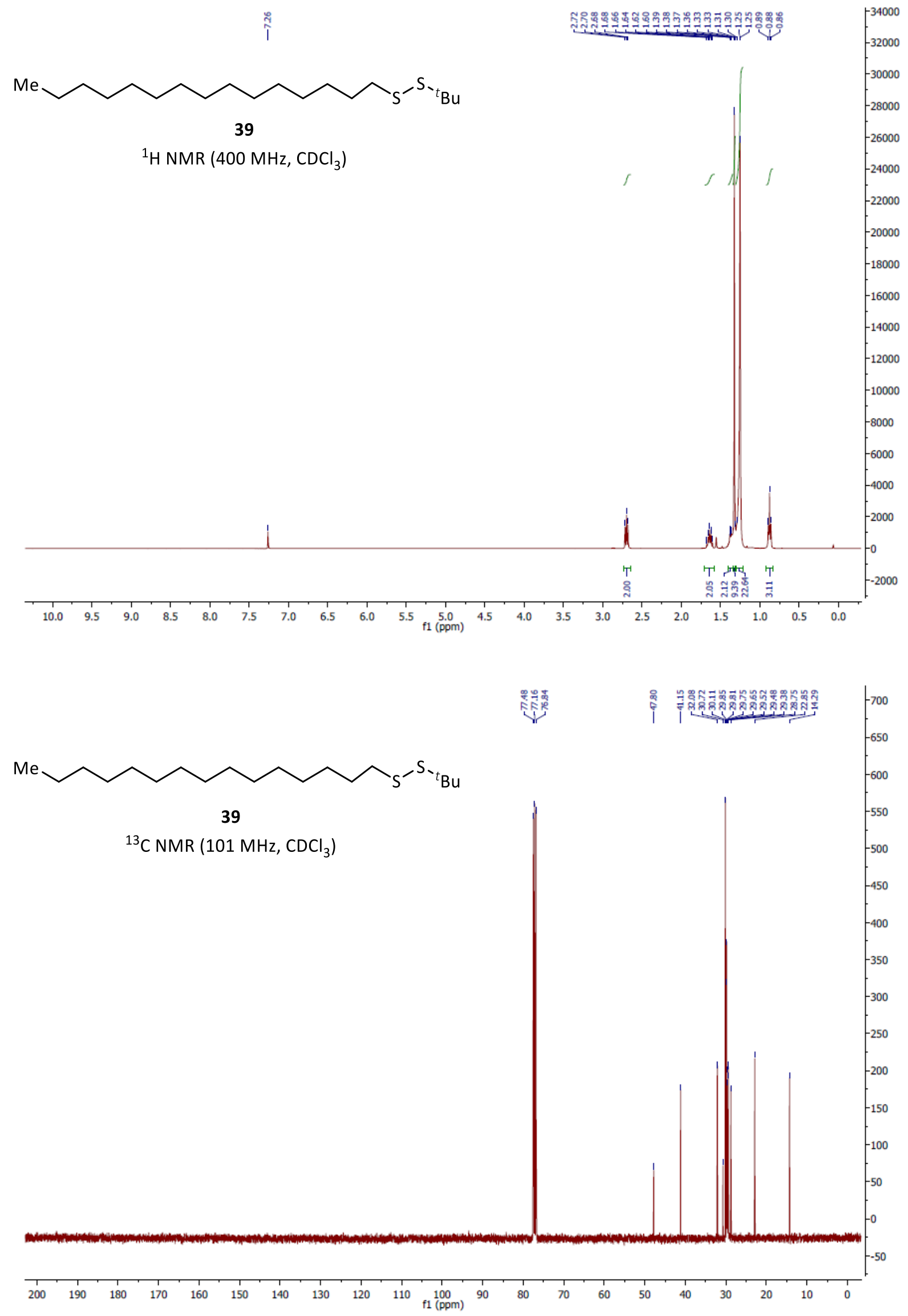


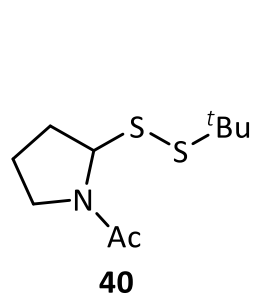

${ }^{1} \mathrm{H}$ NMR $\left(400 \mathrm{MHz}, \mathrm{CDCl}_{3}\right)$

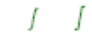

\section{1.)}

les

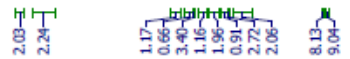

$\begin{array}{rlllllllllllllllllllllll}1 & 10.0 & 9.5 & 9.0 & 8.5 & 8.0 & 7.5 & 7.0 & 6.5 & 6.0 & 5.5 & \begin{array}{c}1 \\ 1.0\end{array} & 4.5 & 4.0 & 3.5 & 3.0 & 2.5 & 2.0 & 1.5 & 1.0 & 0.5 & 0.0 & 1\end{array}$

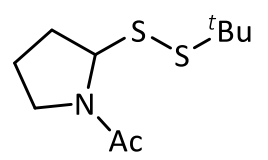

40

${ }^{13} \mathrm{C}$ NMR $\left(101 \mathrm{MHz}, \mathrm{CDCl}_{3}\right)$

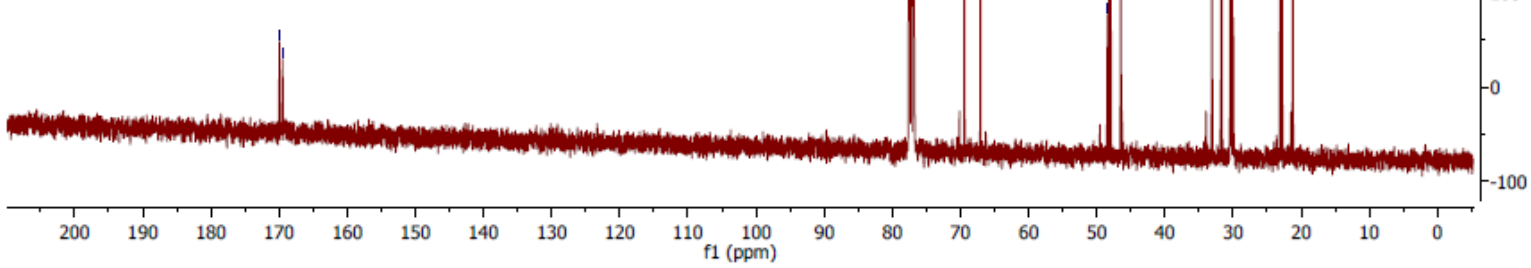



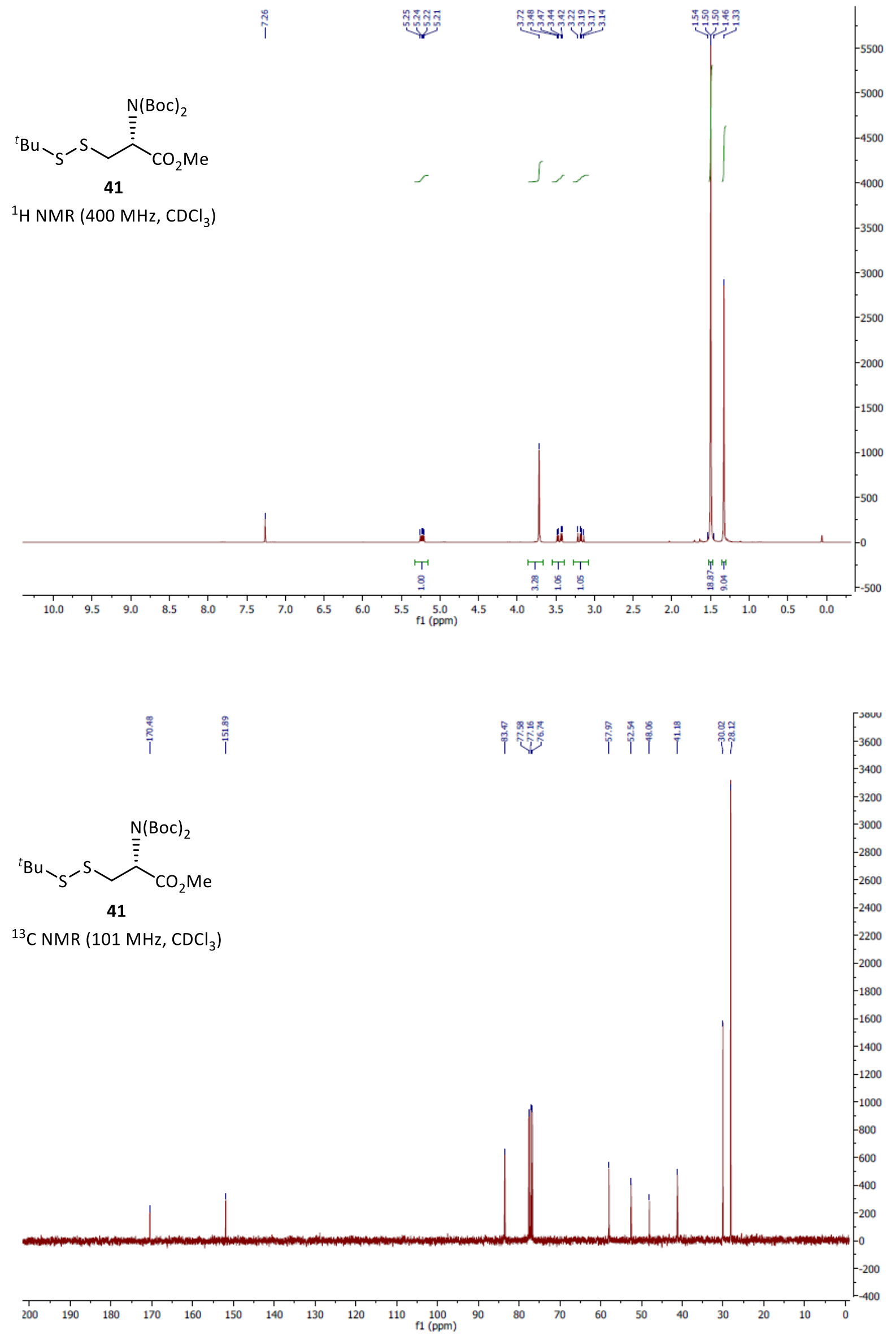

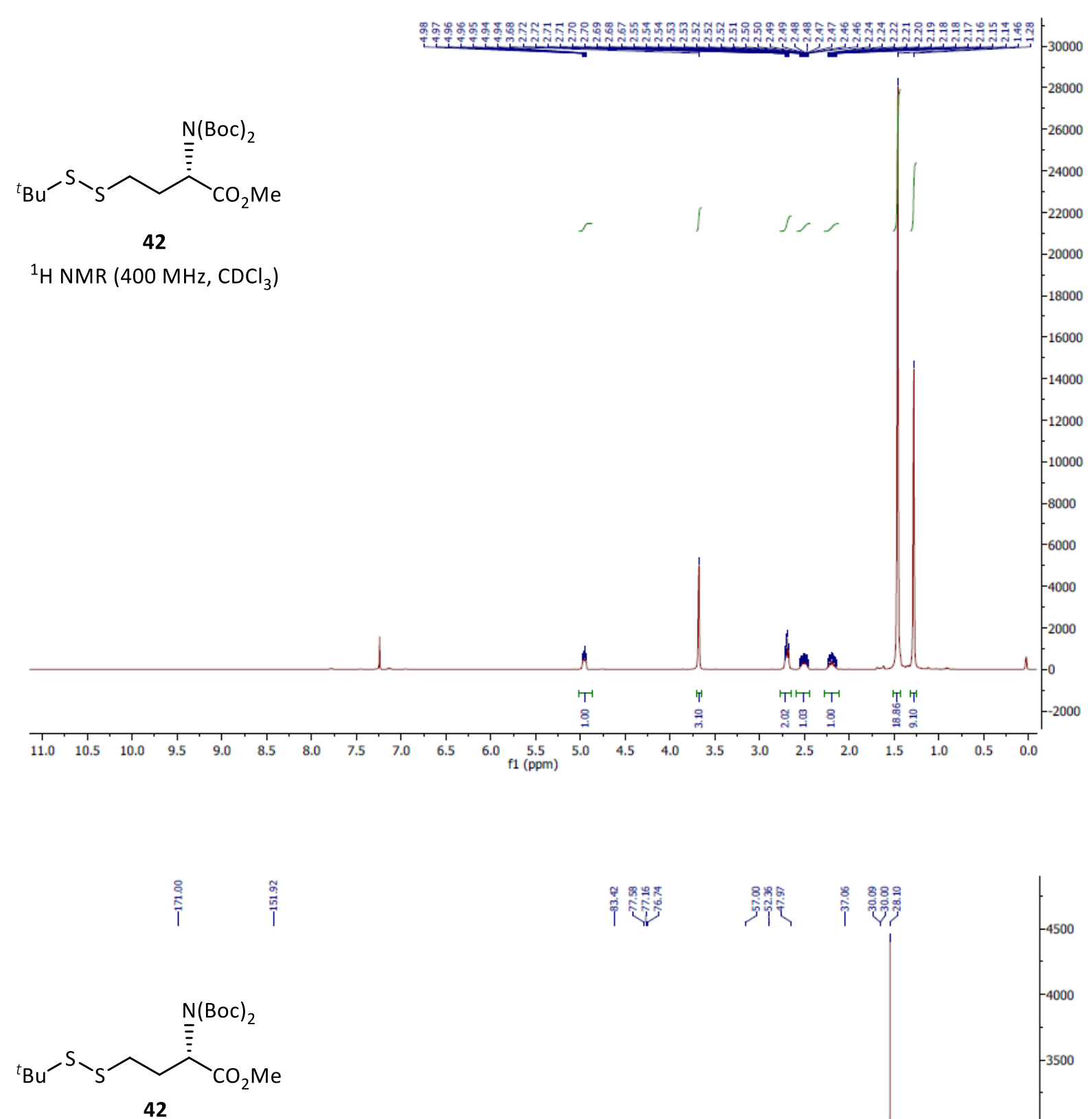

4500

${ }^{13} \mathrm{C}$ NMR $\left(101 \mathrm{MHz}, \mathrm{CDCl}_{3}\right)$

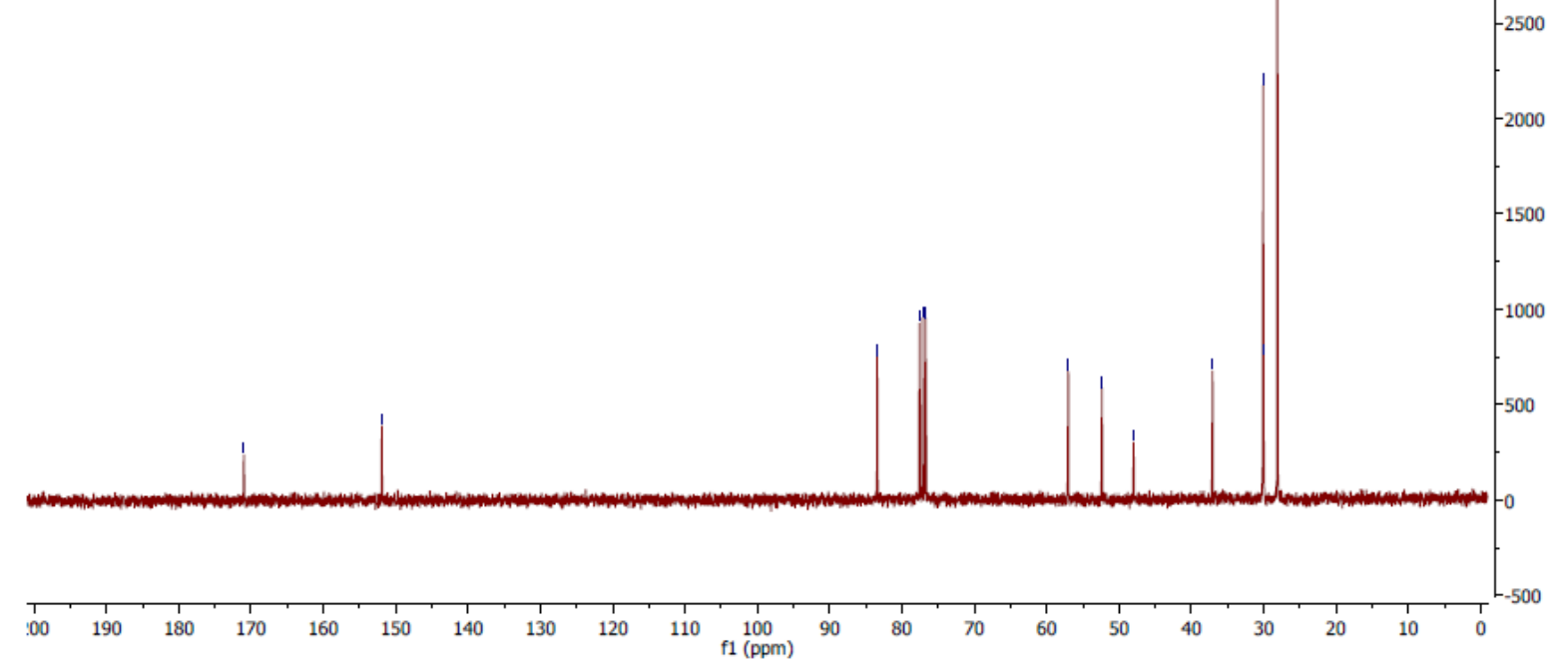



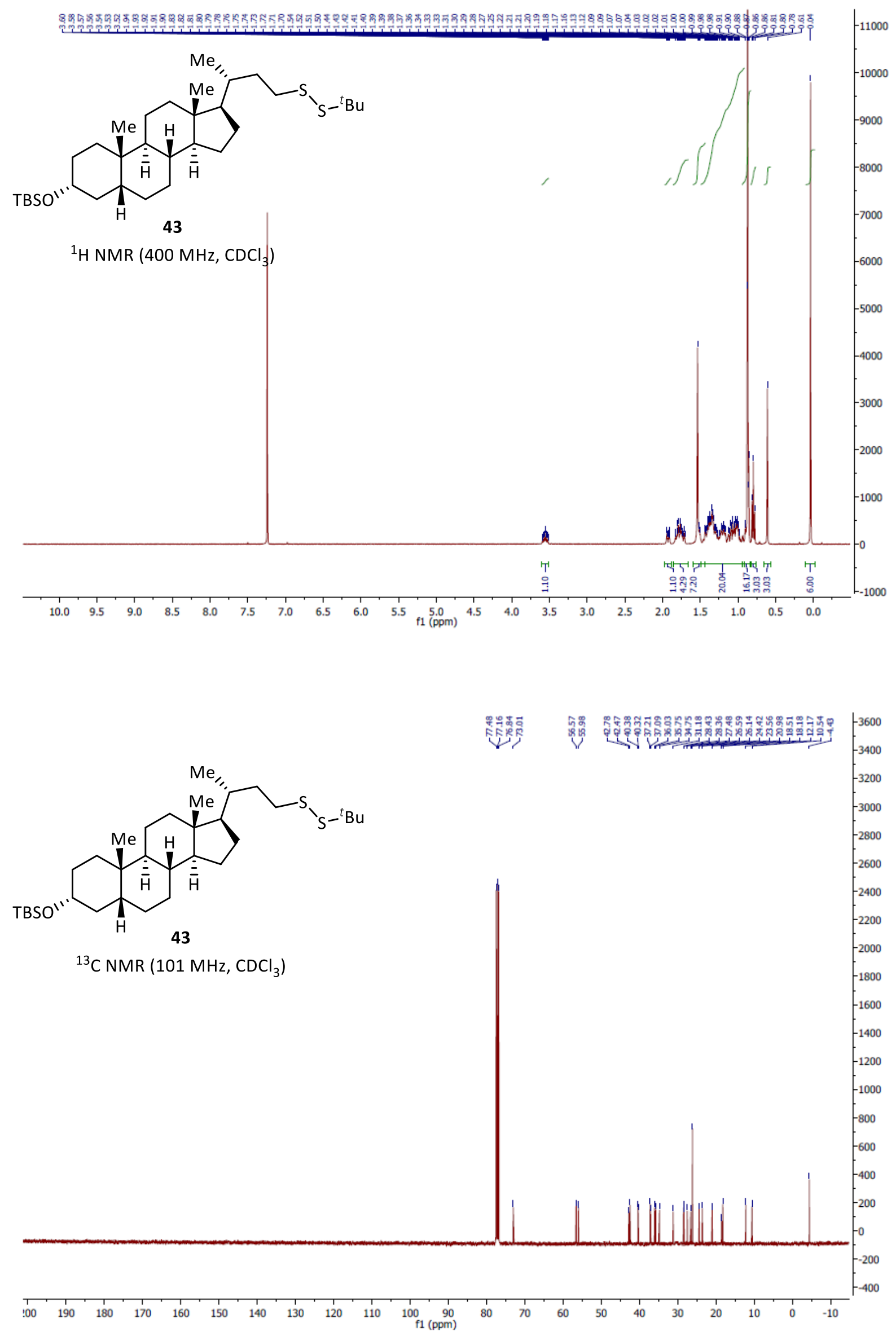
<smiles>C=C/C=C\CCCSSC(C)(C)c1ccccc1</smiles>

47<smiles>c1ccccc1</smiles>

${ }^{1} \mathrm{H}$ NMR $\left(400 \mathrm{MHz}, \mathrm{CDCl}_{3}\right)$
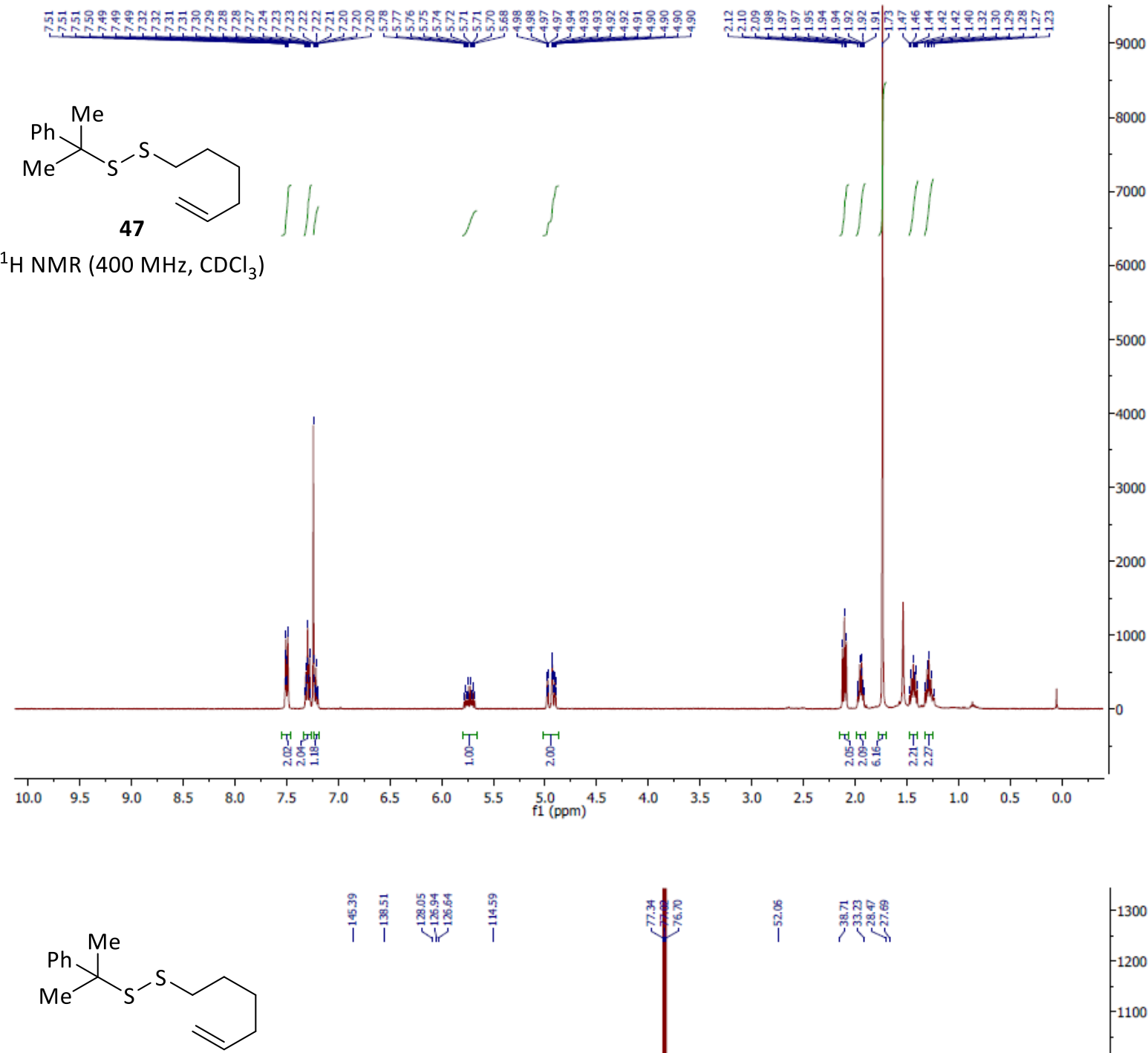

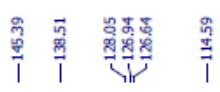

47

$\left.{ }^{13} \mathrm{C} \mathrm{NMR} \mathrm{(101} \mathrm{MHz,} \mathrm{CDCl}_{3}\right)$

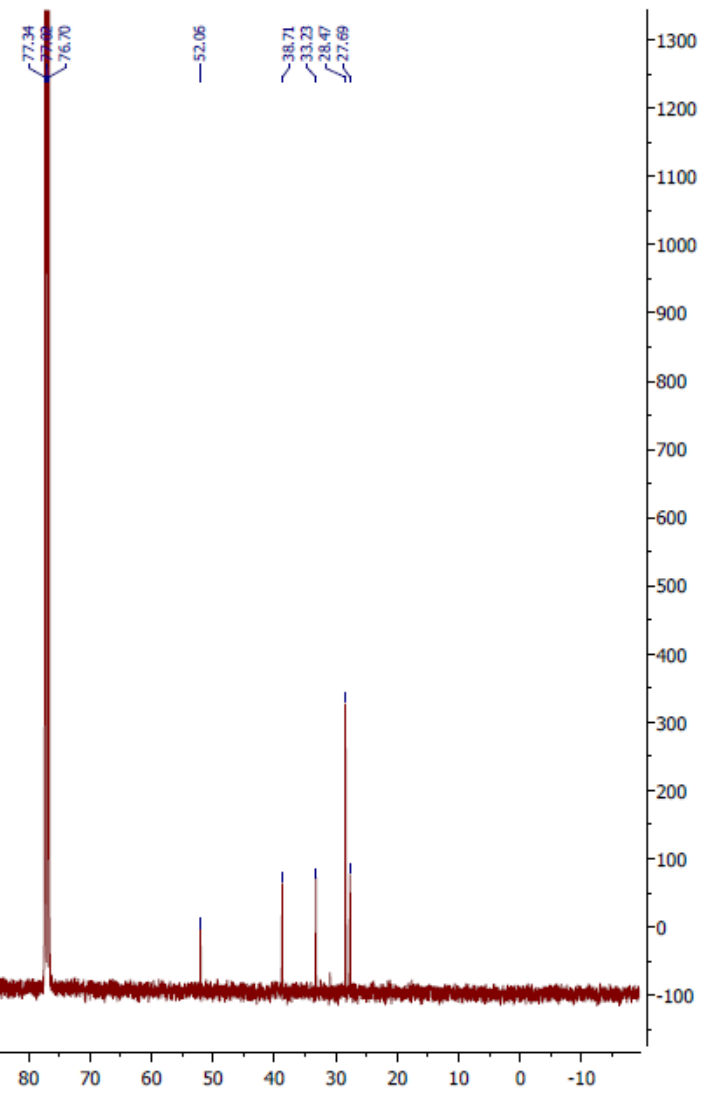



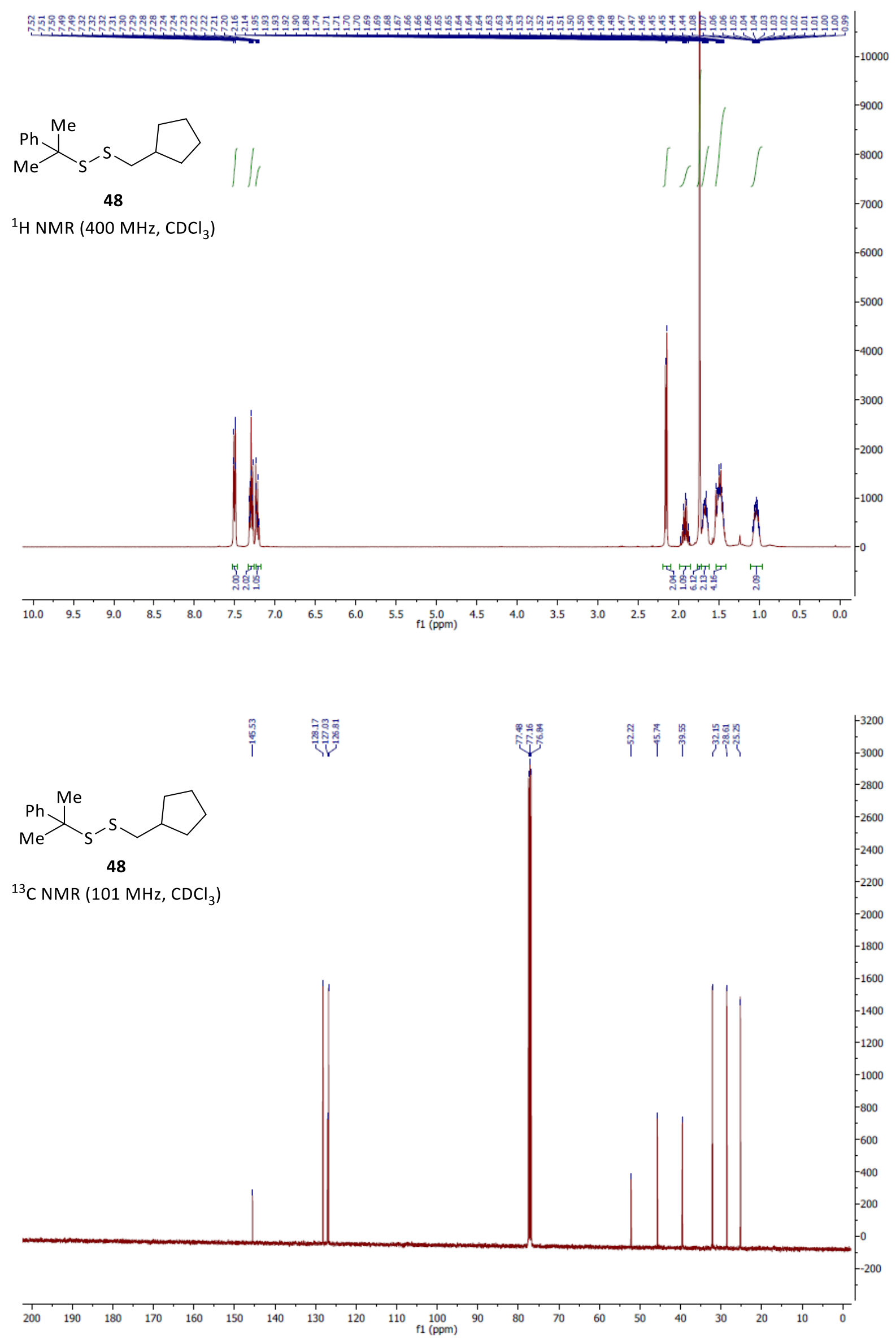

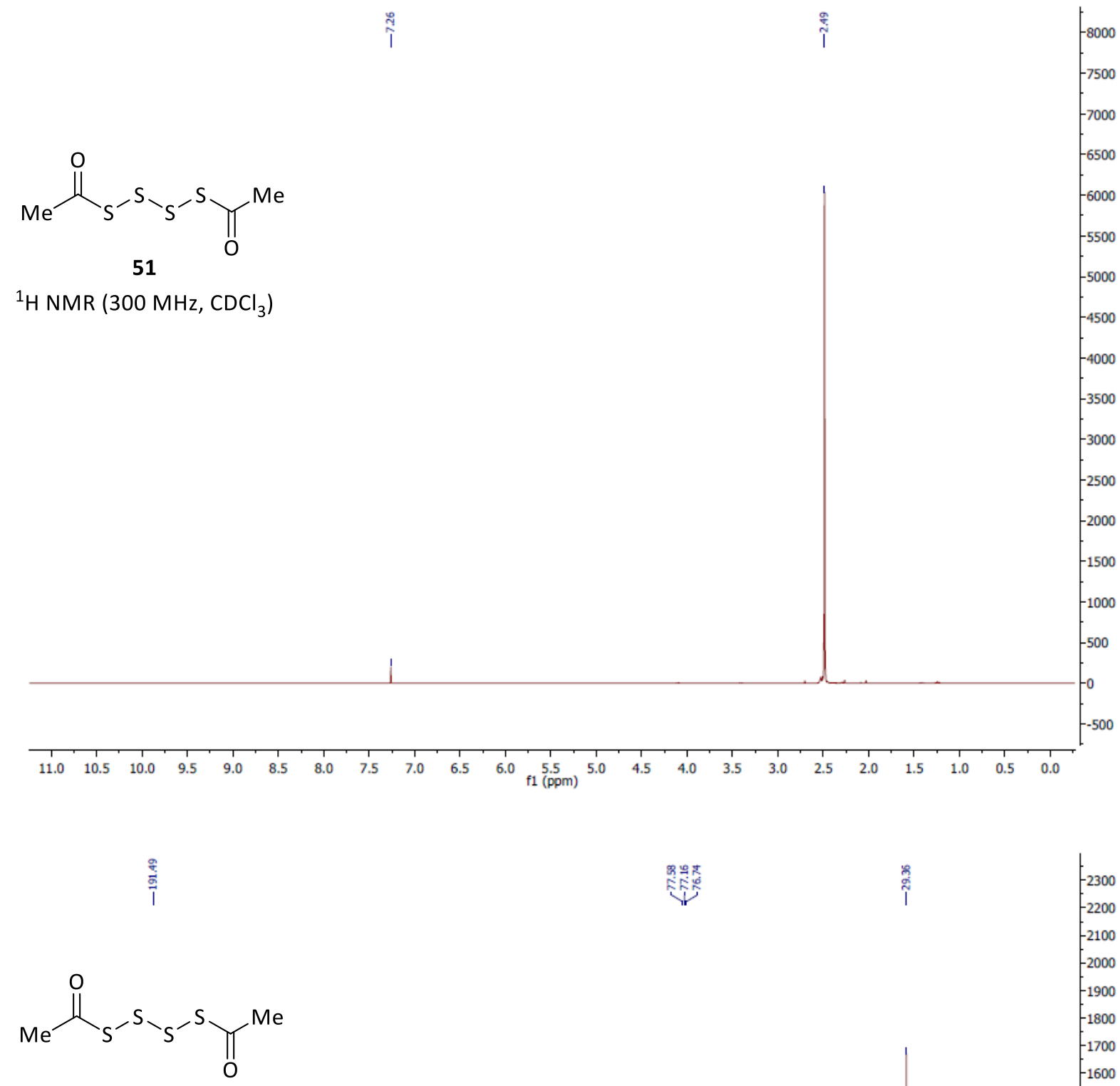

51

${ }^{13} \mathrm{C}$ NMR (75 MHz, $\mathrm{CDCl}_{3}$ )

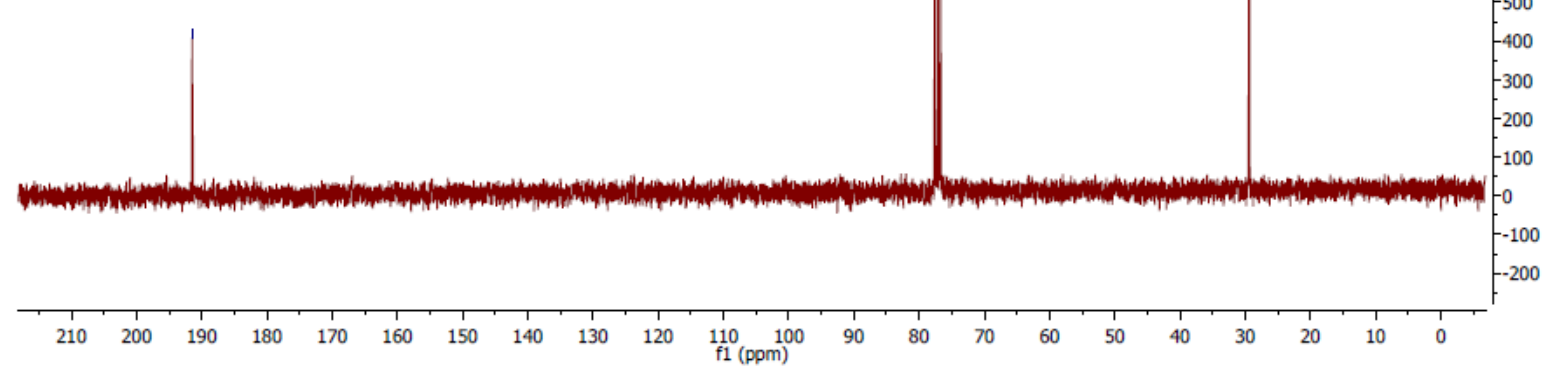



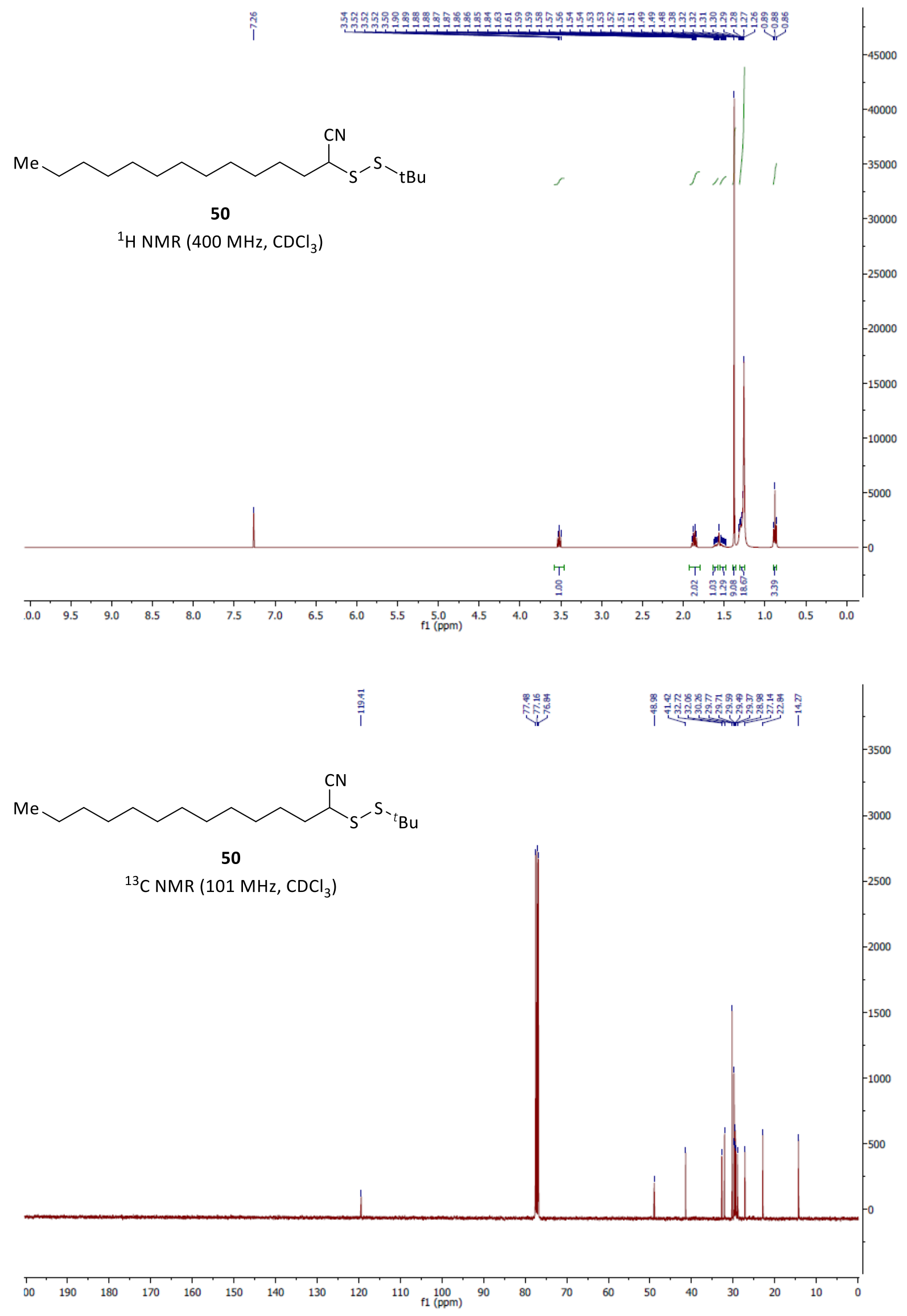

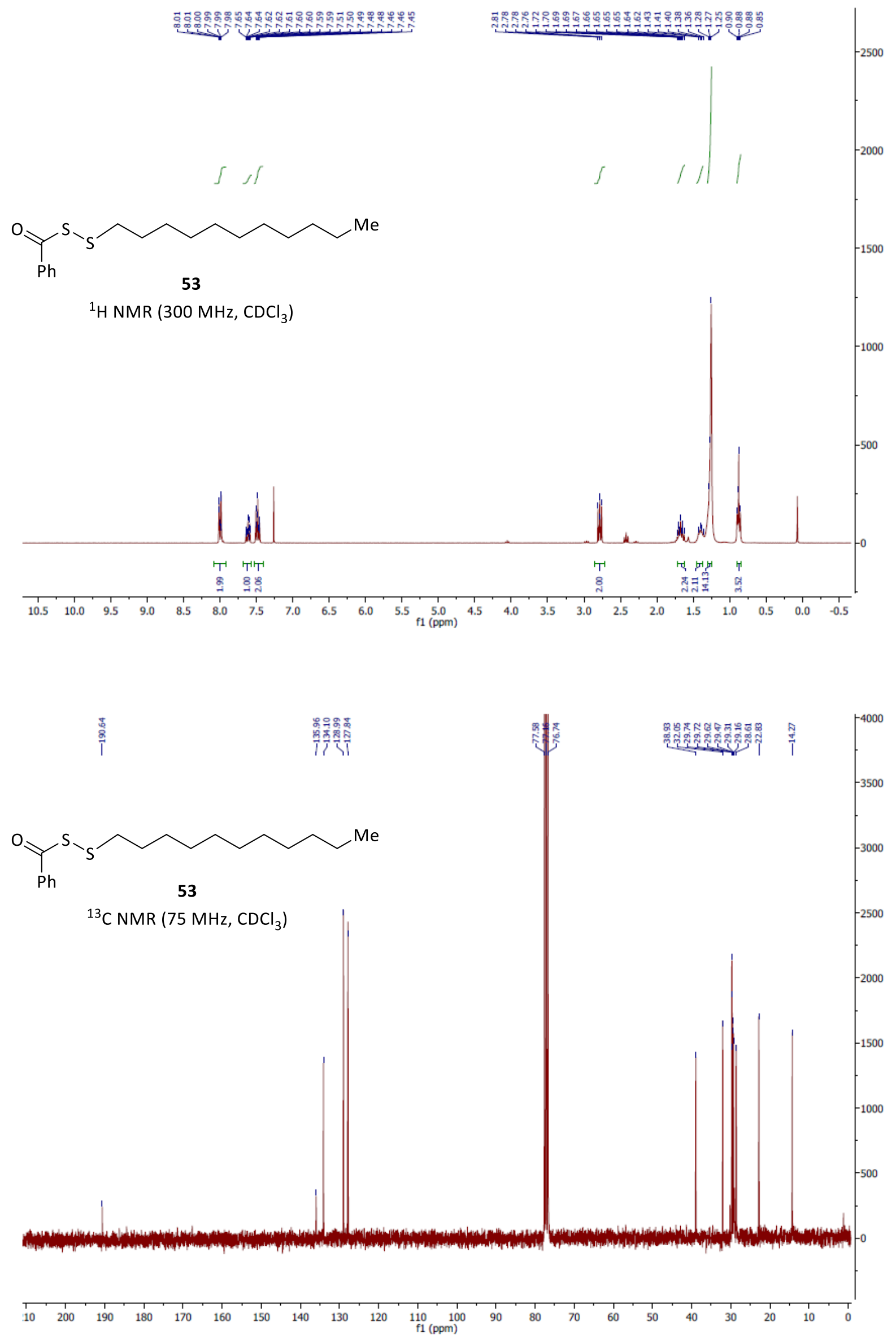

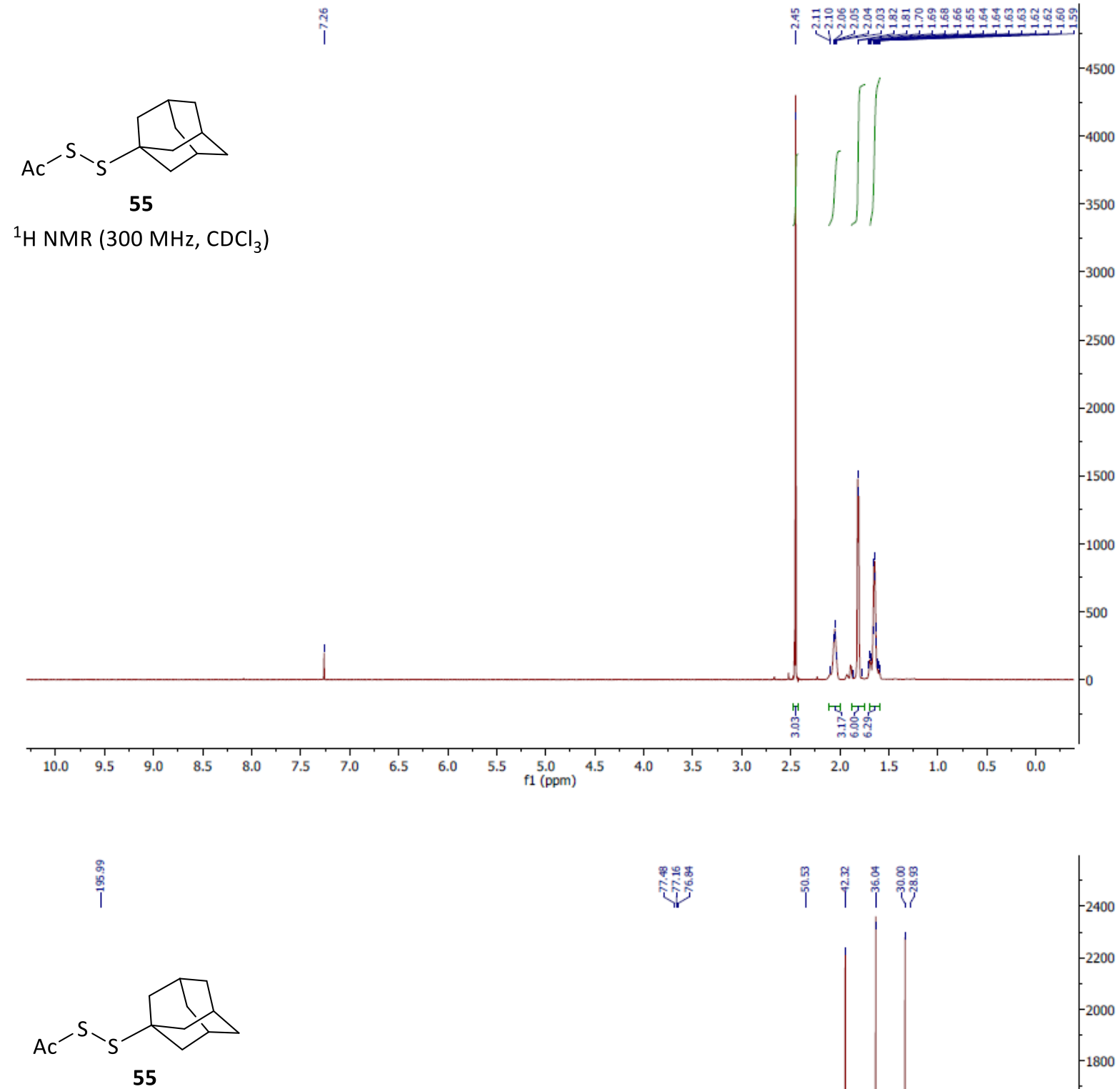

${ }^{13} \mathrm{C}$ NMR (101 MHz, $\mathrm{CDCl}_{3}$ )

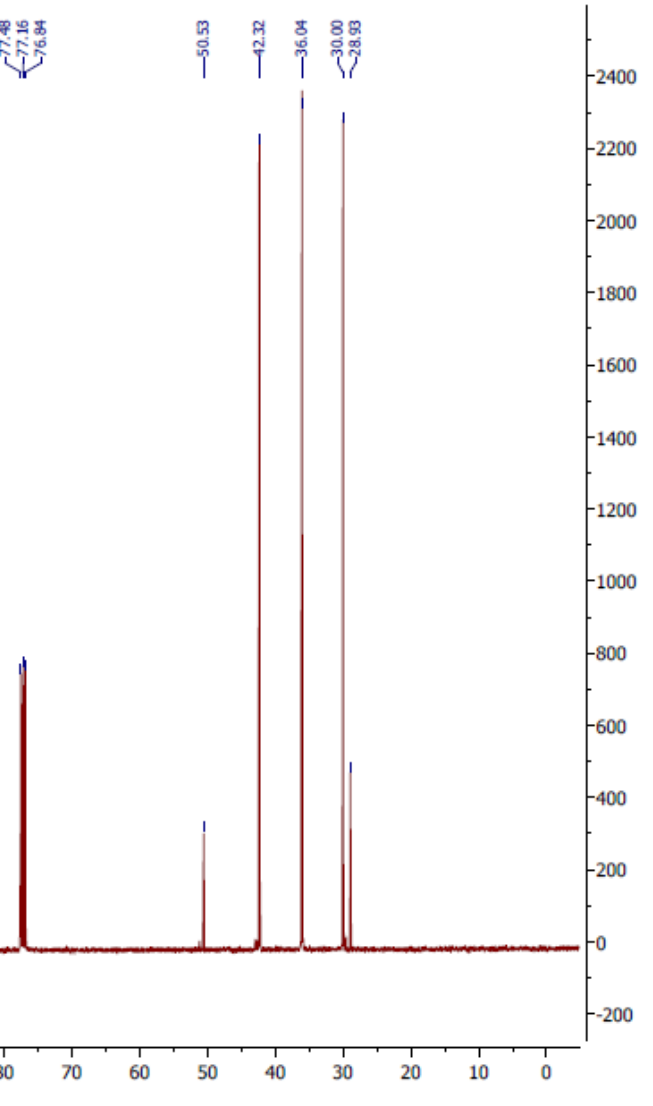



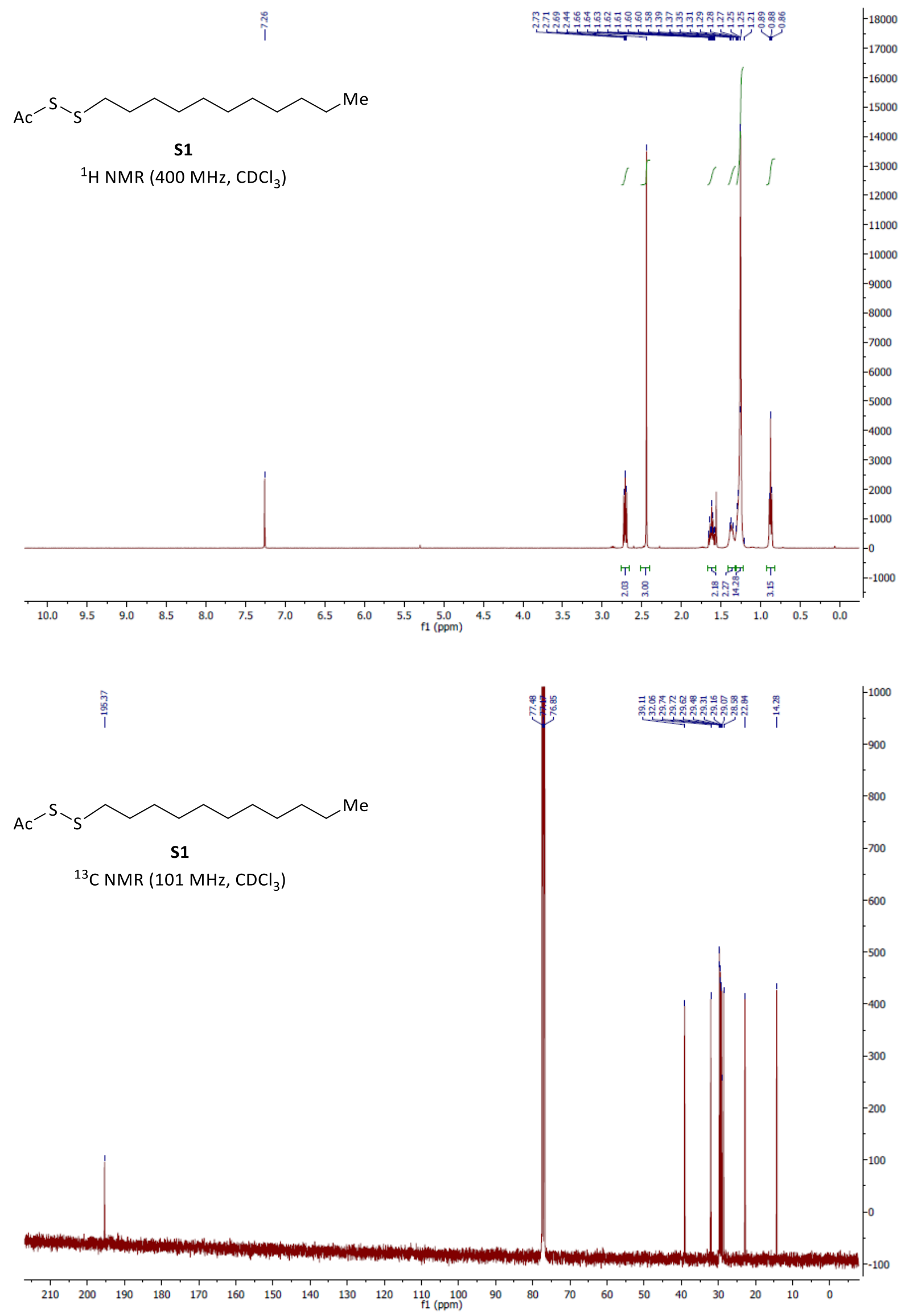


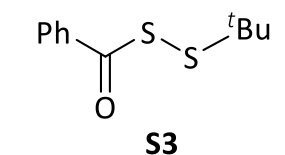

S3
${ }^{1} \mathrm{H}$ NMR $\left(400 \mathrm{MHz} \mathrm{CDCl}_{3}\right)$
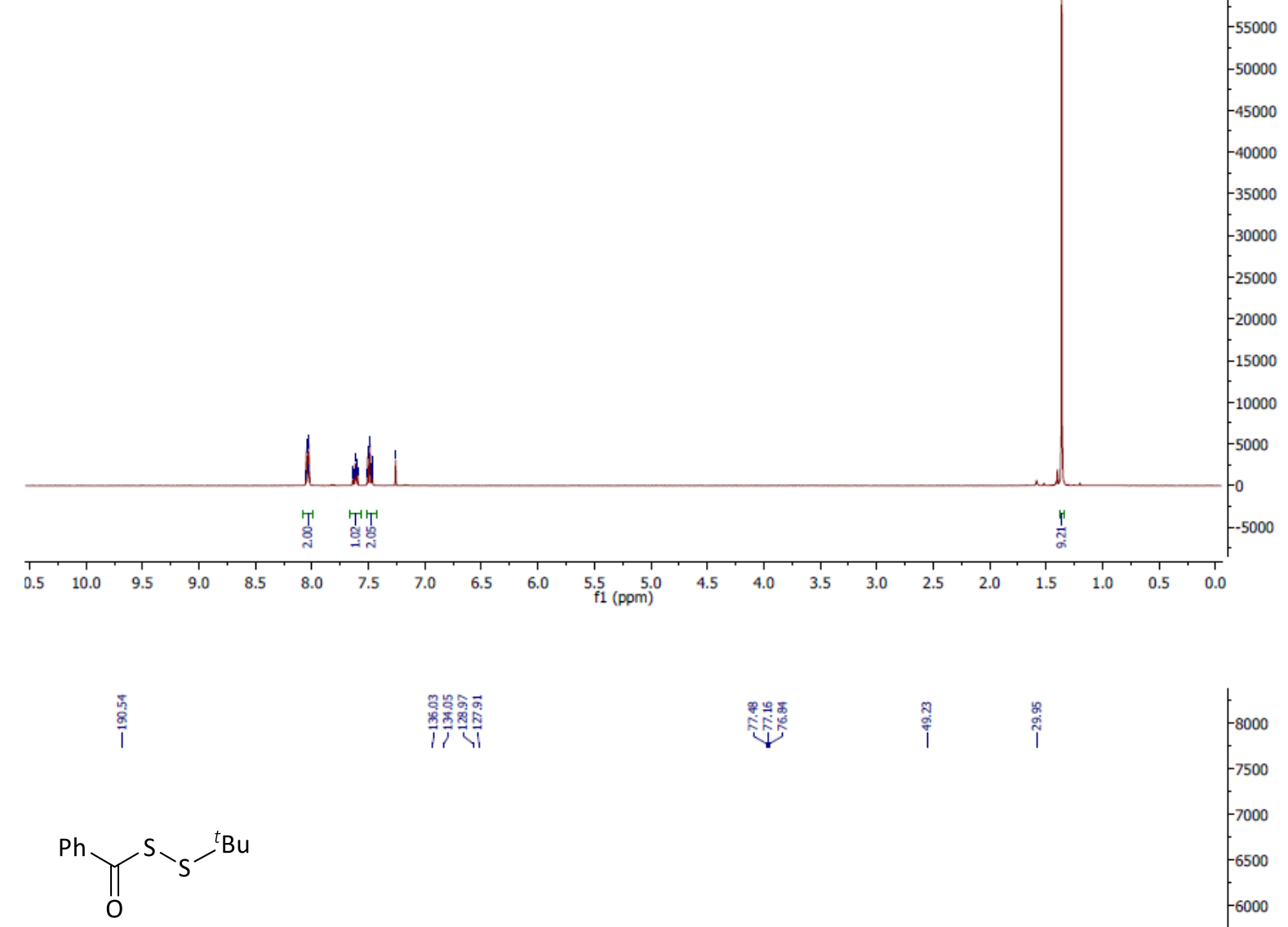

S3

${ }^{13} \mathrm{C}$ NMR $\left(101 \mathrm{MHz}, \mathrm{CDCl}_{3}\right)$

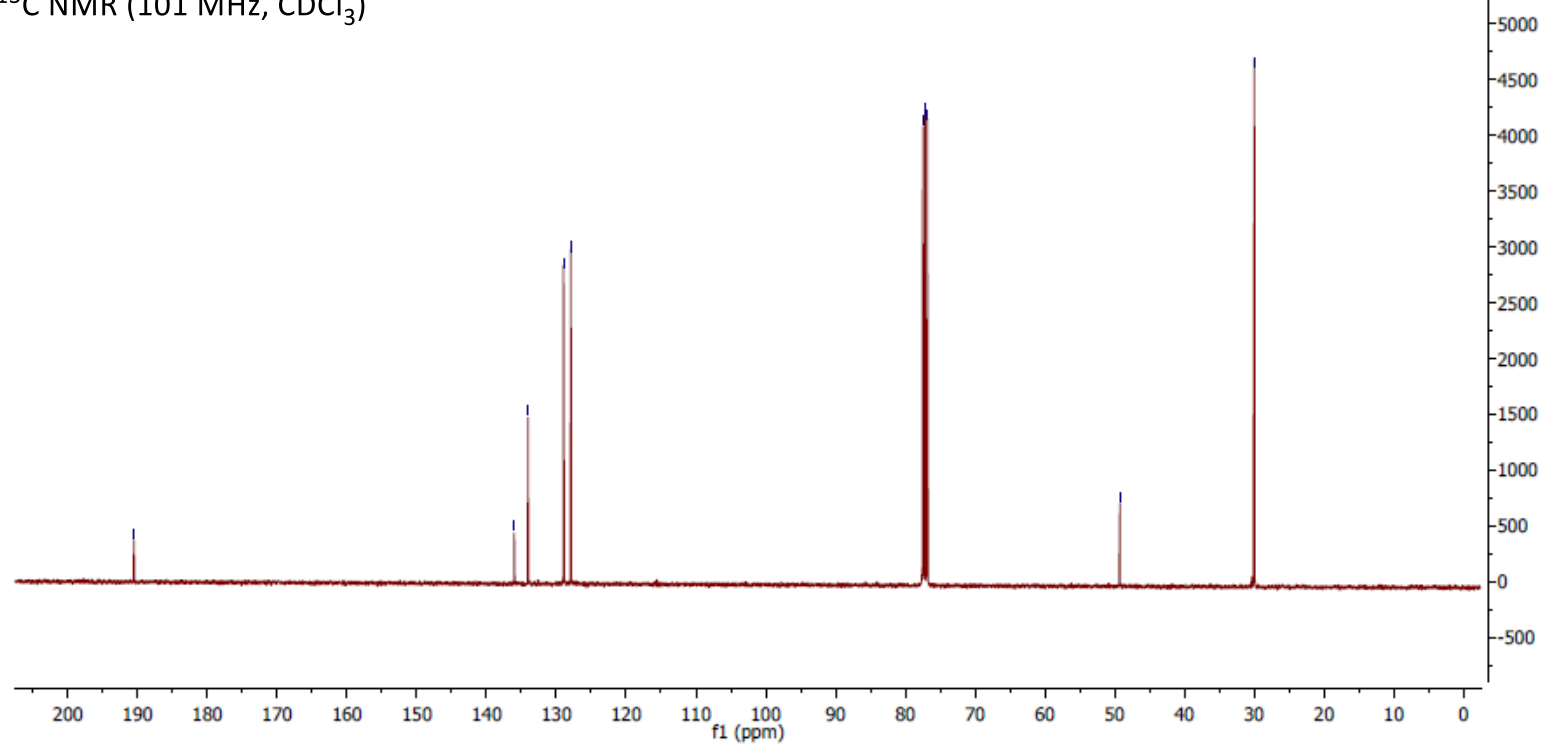

